\title{
Singularly Perturbed Oscillators with Exponential Nonlinearities
}

\section{S. Jelbart ${ }^{1} \mathbb{D} \cdot$ K. U. Kristiansen ${ }^{2} \cdot$ P. Szmolyan ${ }^{3} \cdot$ M. Wechselberger ${ }^{4}$}

Received: 23 December 2019 / Revised: 22 June 2021 / Accepted: 2 July 2021 /

Published online: 19 July 2021

(c) The Author(s) 2021

\begin{abstract}
Singular exponential nonlinearities of the form $e^{h(x) \epsilon^{-1}}$ with $\epsilon>0$ small occur in many different applications. These terms have essential singularities for $\epsilon=0$ leading to very different behaviour depending on the sign of $h$. In this paper, we consider two prototypical singularly perturbed oscillators with such exponential nonlinearities. We apply a suitable normalization for both systems such that the $\epsilon \rightarrow 0$ limit is a piecewise smooth system. The convergence to this nonsmooth system is exponential due to the nonlinearities we study. By working on the two model systems we use a blow-up approach to demonstrate that this exponential convergence can be harmless in some cases while in other scenarios it can lead to further degeneracies. For our second model system, we deal with such degeneracies due to exponentially small terms by extending the space dimension, following the approach in Kristiansen (Nonlinearity 30(5): 2138-2184, 2017), and prove-for both systems-existence of (unique) limit cycles by perturbing away from singular cycles having desirable hyperbolicity properties.
\end{abstract}

Keywords Singular perturbations · Non-smooth systems $\cdot$ Blow-up method $\cdot$ Exponential asymptotics $\cdot$ Relaxation oscillations

\section{Introduction}

Exponential nonlinearities arise in many different areas of mathematical modelling. In electronic oscillators, for example, the Ebers-Moll model for an NPN transistor provides an exponential relationship between the 'emitter current' and the 'base-emitter voltage'. See

$凶$ S. Jelbart

jelbart@ma.tum.de

1 Department of Mathematics, Technical University of Munich, 85748 Garching, Bavaria, Germany

2 Department of Applied Mathematics and Computer Science, Technical University of Denmark, 2800 Kgs. Lyngby, Denmark

3 Institute for Analysis and Scientific Computing, Vienna University of Technology, Wiedner Hauptstraße 8-10, 1040 Vienna, Austria

4 School of Mathematics \& Statistics, University of Sydney, Camperdown, NSW 2006, Australia 
[7,11]. Also, in chemical kinetics, the reaction rates are, by the Arrhenius equation, exponential functions of the temperature. Frequently, the temperature is assumed constant in such models, but in systems where large temperature variations occur (e.g. in explosions), the resulting exponential nonlinearity becomes important for the dynamics. Similar nonlinearities appear in other settings when the effect of temperature becomes important, see e.g. $[2,8]$ for exponential nonlinearities in plastic deformation. In the related area of friction, exponential nonlinearities also play an important role, for example in rate-and-state friction laws, see $[4,5,32,33]$ and $[1,21,30]$ for dynamical studies of such models. Although these friction models were first derived from experiments, the exponential nonlinearities have later been connected to Arrhenius process resulting from breaking atomic bonds at the atomic level [31]. Sometimes modellers also introduce exponentials more heuristically, for example when regularizing a switch by a tanh-function.

All of the examples of exponential nonlinearities highlighted above, are also examples of (within relevant parameter regimes) singularly perturbed systems. Over the past decades, these type of systems have been successfully described by geometric singular perturbation theory (GSPT) and blow-up, see e.g. [6,9,17,25], but singular exponential nonlinearities like $e^{h(x) \epsilon^{-1}}$, with essential singularities along $h(x)=0$ as $\epsilon \rightarrow 0$, have traditionally been seen as an obstacle to such analysis.

The problem is two-fold. Firstly, such systems approach piecewise smooth systems (upon proper normalizations) as $\epsilon \rightarrow 0$, having very different behaviour for $h(x)>0$ and $h(x)<0$. The mathematical analysis of smooth systems with non-smooth singular limits via GSPT and blow-up is currently an active area of research, see e.g. [13,19,23,24]. Secondly, for systems with nonlinearities of the form $e^{h(x) \epsilon^{-1}}$ the convergence of the smooth system to its nonsmooth counterpart happens at an exponential rate. Whereas this exponential convergence can be harmless in some cases, it can also lead to further degeneracies due to 'exponential loss of hyperbolicity'. We will demonstrate this through the study of two prototypical systems, which we introduce in the following. The usual blowup method [6,25] is adapted to algebraic loss of hyperbolicity, and cannot compensate for these exponential degeneracies. Nevertheless, recently in [20] it was shown how one can modify this approach (basically by extending the space dimension) to deal with these special degeneracies. We will use this modified blowup approach in the present paper.

Finally, it is worthy to remark on the applicability of the methods proposed beyond the scope of the prototypical oscillators considered herein. Both of these oscillators feature exponential nonlinearities of the specific form $e^{x \epsilon^{-1}}$. Nevertheless, such a form is 'typical' in the sense that it may be viewed as a 'local normal form' for a larger class of essential singularities $e^{h(x) \epsilon^{-1}}$, where $h(x)$ is any regular smooth function of the state variable(s) $x$ such that the zero level set $\Sigma=\{h(x)=0\}$ is codimension-1 in state space. All such singularities may be treated using the methods proposed herein, either directly, or following a suitable local coordinate transformation $u=h(x)$.

\subsection{Two Prototypical Oscillators: Hester and Le Corbeiller}

The aim of our paper, is to shed further light on singularly perturbed systems with exponential nonlinearities. We will do so by considering two 'prototypical' singularly perturbed oscillators with exponential nonlinearities: 


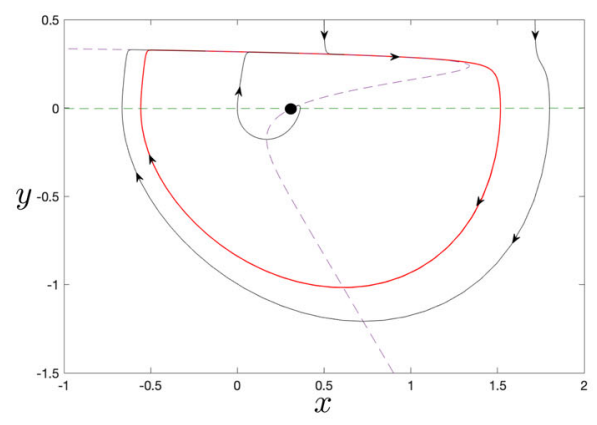

(a)

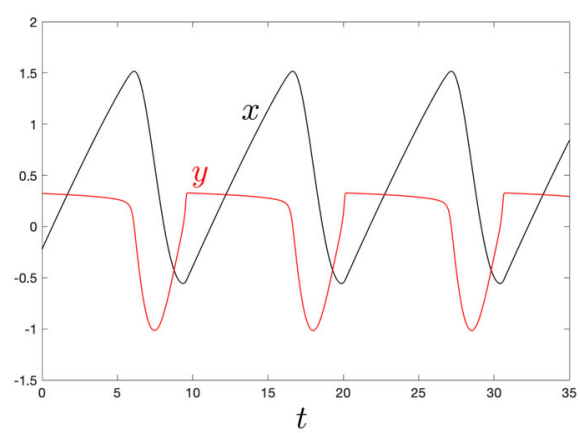

(b)

Fig. 1 In a Phase portrait of (1.1) for the parameter values in (1.3) and $\epsilon=0.1$. A stable limit cycle is shown in red. The repelling equilibrium is marked as a black dot. In $\mathbf{b} x(t)$ and $y(t)$ along the limit cycle shown in a. Also in a The nullclines are dashed and unstable focus is indicated by a black disk (Color figure online)

$$
\text { The Hester system: }\left\{\begin{array}{l}
\dot{x}=y, \\
\dot{y}=-x-2 \gamma y+\mu\left(e^{y \epsilon^{-1}}-\kappa e^{(1+\alpha) y \epsilon^{-1}}\right),
\end{array}\right.
$$

with $\gamma \in(0,1), \alpha>0, \mu>0, \kappa>0$, and

$$
\text { The Le Corbeiller system: }\left\{\begin{array}{l}
\dot{x}=y+a, \\
\dot{y}=-x+b y\left(2-e^{y \epsilon^{-1}}\right),
\end{array}\right.
$$

with $b \in(0,1), a>0$. In both systems, $0<\epsilon \ll 1$ is the singular perturbation parameter.

The system in (1.1) is a model of a transistor oscillator, see [11], based on the EbersMoll large-signal approximation. In reference to [11], we will refer to (1.1) as the 'Hester' system. The constant $\epsilon^{-1}$ in the exponentials is given by $e / k T$ where $k$ is the Boltzmann constant, $e$ is the magnitude of the electronic charge and $T$ is the temperature in Kelvin. The approximation $0<\epsilon \ll 1$ is therefore valid for sufficiently large temperatures $T$ including room temperatures where $\epsilon \approx 10^{-2}$.

Figure 1 shows the phase portrait and associated oscillations with parameter values

$$
\alpha=0.5, \mu=0.4, \kappa=0.2, \gamma=0.3,
$$

and $\epsilon=0.1$. The observed sharp transitions indicate singular dynamics, even for this 'large' value of $\epsilon$. The oscillations become increasingly slow-fast in kind with decreasing values of $\epsilon$, as shown in Fig. 2, which shows the phase portrait and associated oscillations with the same parameter values (1.3), except with $\epsilon=0.01$. The oscillations in Figs. 1 and 2 are known as two-stroke relaxation oscillations, by reference to the two distinct components to the oscillation; similar oscillations have been considered in the context of GSPT in [16].

Regarding the motivation for the system in (1.2), we first point out that for $a=0$ the $\epsilon$ can be scaled out by setting $x=\epsilon x_{2}$, such that

$$
\ddot{x}_{2}+x_{2}+\mu \dot{x}_{2}\left(e^{\dot{x}_{2}}-2\right)=0 \text {, }
$$

when writing the system as a second order equation. This equation appears in [29, Eq. (25)] as an example of a simple system of 'electric-oscillator-type' exhibiting two-stroke oscillation. Within the framework of electronic oscillators, we may therefore consider (1.2) with $a>0$ as a forced version of (1.4) (by analogy with the 'forced van der Pol oscillator'). In reference 


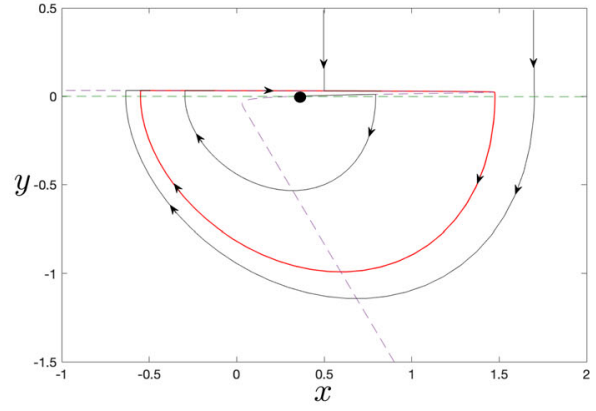

(a)

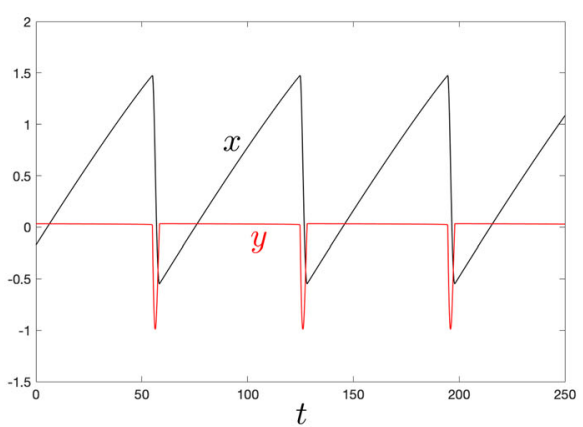

(b)

Fig. 2 In a Phase portrait of (1.1) for the parameter values in (1.3) and $\epsilon=0.01$. A stable limit cycle is shown in red. In $\mathbf{b} x(t)$ and $y(t)$ along the limit cycle shown in $\mathbf{a}$

to [29], we will refer to (1.2) as the 'Le Corbeiller' system. As in Figs. 1 and 2, the phase portrait and associated oscillations for (1.2) are shown in Figs. 3 and 4, for parameter values

$$
a=1, b=0.25 \text {, }
$$

$\epsilon=0.1$ in Fig. 3, and $\epsilon=0.01$ in Fig. 4. As with the Hester system (1.1), sharp transitions between distinct components of the oscillations indicates singular dynamics even for the 'large' $\epsilon$ value in Fig. 3. In contrast to the Hester system, however, $\dot{x}=a>0$ along the (noninvariant) set defined by $y=0$ for (1.2) and - as a result — the oscillations, spending a fraction of their time near this set, do not become slow-fast in kind with decreasing $\epsilon$. Rather it appears that the period has a well-defined limit as $\epsilon \rightarrow 0$. Nevertheless, the 'singular' nature of the oscillations does become more pronounced as $\epsilon \rightarrow 0$, insofar as the transition between the two distinct components of the oscillation becomes sharper.

\subsection{Main Results}

We prove existence of limit cycles for (1.1) and (1.2) for all $0<\epsilon \ll 1$ using a combination of GSPT and the blow-up method adapted in [20] for the study of degeneracies caused by exponential nonlinearities. We present these results in the following, considering each system separately.

Remark 1.1 While systems (1.1) and (1.2) seem similar in nature, the level of difficulty to analyse them using the GSPT toolbox is remarkably different.

\subsubsection{The Hester System}

Due to the singular exponential nonlinearity $e^{y \epsilon^{-1}}$, system (1.1) has no limit for $\epsilon \rightarrow 0$ for $y>0$. This problem can be circumvented by appealing to the notion of topological equivalence and applying a time transformation

$$
d t_{1}=\left(1+e^{(1+\alpha) y \epsilon^{-1}}\right) d t
$$




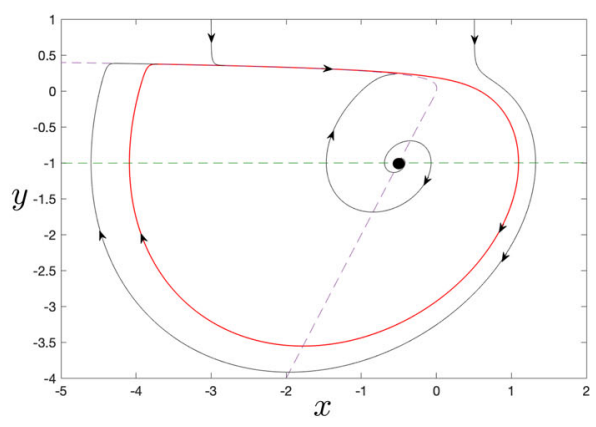

(a)

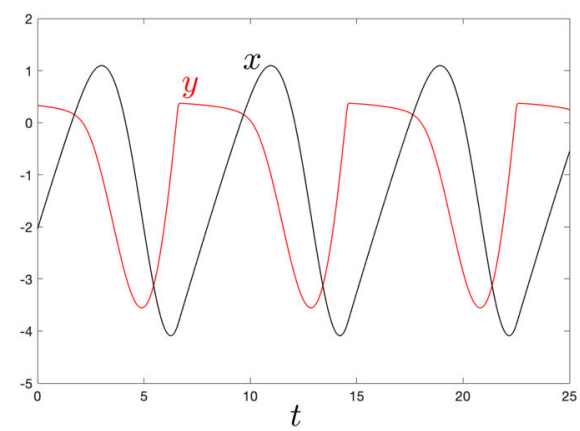

(b)

Fig. 3 In a Phase portrait of (1.2) for the parameter values in (1.5) and $\epsilon=0.1$. The attracting limit cycle is shown in red, the repelling equilibrium is marked by a black dot. Even for this large value of $\epsilon$ the system clearly displays a form of multi-scale dynamics, e.g. note the rather sharp corner of the limit cycle. In $\mathbf{b} x(t)$ and $y(t)$ along the limit cycle shown in a (Color figure online)

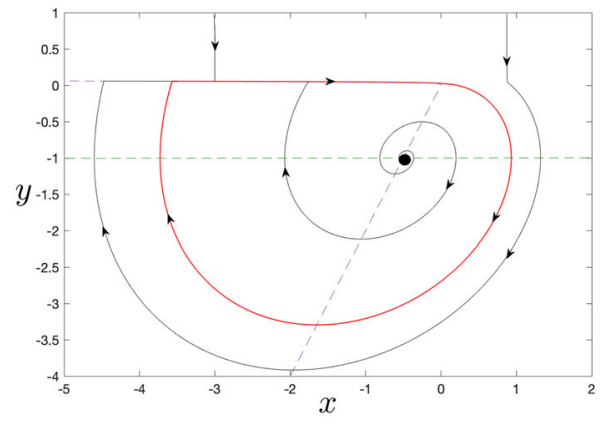

(a)

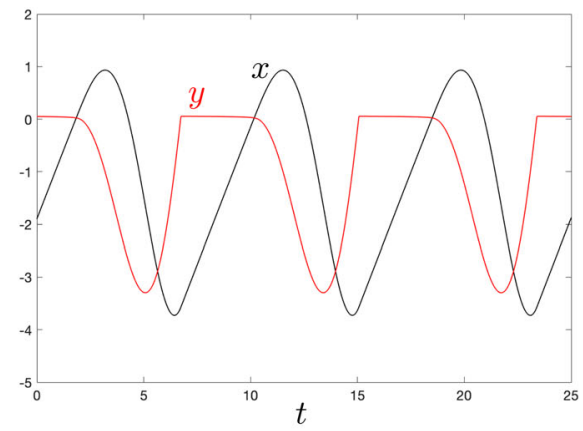

(b)

Fig. 4 In a Phase portrait of (1.2) for the parameter values in (1.5) and $\epsilon=0.01$. A stable limit cycle is shown in red. A sharp corner in the limit cycle is now clearly visible. In $\mathbf{b} x(t)$ and $y(t)$ along the limit cycle shown in a (Color figure online)

which gives

$$
\begin{aligned}
& \dot{x}=\frac{y}{1+e^{(1+\alpha) y \epsilon^{-1}}}, \\
& \dot{y}=\frac{-x-2 \gamma y+\mu e^{y \epsilon^{-1}}}{1+e^{(1+\alpha) y \epsilon^{-1}}}-\frac{\kappa \mu e^{(1+\alpha) y \epsilon^{-1}}}{1+e^{(1+\alpha) y \epsilon^{-1}}},
\end{aligned}
$$

where with a slight abuse of notation the overdot denotes differentiation with respect to the new time $t_{1}$. For any $\epsilon>0$, this corresponds to a smooth transformation of time; (1.1) and (1.7) therefore have the same orbits for any $\epsilon>0$.

Remark 1.2 Viewed less abstractly, the time transformation defined by (1.6) corresponds to a multiplication of the vector-field by the strictly positive function $\left(1+e^{(1+\alpha) y \epsilon^{-1}}\right)^{-1}$. This leaves all orbits unchanged. Such space dependent rescalings together with the blow-up method are commonly used in GSPT, see e.g. $[18,19,26]$ and will also be used frequently in the present paper. 
Clearly, the time transformation defined by (1.6) is singular for $\epsilon=0$, but it has the advantage that the new system (1.7) has a well-defined pointwise limit as $\epsilon \rightarrow 0$ for any $y \neq 0$. In fact, in this limit we obtain the piecewise smooth (PWS) system:

$$
\begin{aligned}
& \dot{x}=0, \\
& \dot{y}=-\kappa \mu,
\end{aligned}
$$

for $y>0$ and

$$
\begin{aligned}
& \dot{x}=y, \\
& \dot{y}=-x-2 \gamma y,
\end{aligned}
$$

for $y<0$, the dynamics of which we sketch in Fig. 5. The discontinuity set $\Sigma=\{y=0\}$ is called the switching manifold in the PWS literature [3]. Given $\gamma \in(0,1)$, system (1.9) for $y<0$ has a stable focus at $(x, y)=(0,0)$ which is on the switching manifold $\Sigma=\{y=0\}$. In the PWS literature, this situation is known as a boundary focus, see [12,28] or the recent papers $[14,15]$ on boundary singularities in smooth systems with nonsmooth limits. Under the forward flow of (1.8) or (1.9), respectively, every point with $y>0$ or $y<0$ will reach $y=0$ in finite time. The case $\gamma \geq 1$ for which $(0,0)$ is a stable node is also interesting (though not considered in [11]); this is discussed in Sect. 7.

Frequently, in PWS systems one prescribes a Filippov vector-field $[3,10]$ on $\Sigma$ to have a well-defined forward flow. However, since $\dot{x}=0$ on $y=0$ for both (1.8) and (1.9), the Filippov system is completely degenerate on $\Sigma$, consisting entirely of (pseudo-)equilibria $[3,28]$. Our analysis of the Hester problem will reveal a slow flow near the switching manifold $\Sigma$ for all $0<\epsilon \ll 1$ (see Lemma 1.4), and allow us to define a singular relaxation cycle

$$
\Gamma_{0}=\Gamma_{1} \cup \Gamma_{2} .
$$

Here $\Gamma_{1}$ is an orbit segment (a fast jump) of (1.9), obtained by flowing the uniquely identified jump-off point $\left(x_{j}, 0\right) \in \Sigma$ with

$$
x_{j}=\frac{\mu \alpha}{(1+\alpha)^{(1+\alpha) / \alpha} \kappa^{1 / \alpha}},
$$

forward until the first return to $\Sigma$ at the drop point $\left(x_{d}, 0\right)$ with $x_{d}=x_{d}\left(x_{j}\right)<0$, whereas $\Gamma_{2}$ is a 'slow orbit' segment on $\Sigma$ connecting $\left(x_{d}, 0\right)$ with $\left(x_{j}, 0\right)$; see Fig. 5 . The existence of a unique value $x_{j}$ as given by (1.10) follows from the existence of a regular fold [25] of a critical manifold identified in rescaled coordinates with $y=\epsilon y_{2}$, i.e. in the 'switching layer'; details are deferred to Sect. 2.1.

Our main result on the Hester system is the following theorem.

Theorem 1.3 Consider the Hester system (1.1) and fix a large ball $B_{r}$ of radius $r$. Suppose that

$$
\kappa(1+\alpha) \in(0,1), \gamma \in(0,1), \mu>0 .
$$

Then there exists an $\epsilon_{0}>0$ such that for all $\epsilon \in\left(0, \epsilon_{0}\right)$, system (1.1) has a unique limit cycle $\Gamma_{\epsilon}$ in $B_{r}$. Furthermore, $\Gamma_{\epsilon}$ is attracting and

$$
\Gamma_{\epsilon} \rightarrow \Gamma_{0},
$$

in Hausdorff-distance, as $\epsilon \rightarrow 0^{+}$.

If $\kappa(1+\alpha)>1$ then no limit cycles exist for $0<\epsilon \ll 1$ in $B_{r}$. 
Fig. 5 Phase portrait of the piecewise smooth system (1.8), (1.9) in black. The switching manifold $\Sigma=\{y=0\}$ is shown in magenta. Under the conditions (1.11), the singular cycle $\Gamma_{0}=\Gamma^{1} \cup \Gamma^{2}$ perturbs to a stable limit cycle by Theorem 1.3

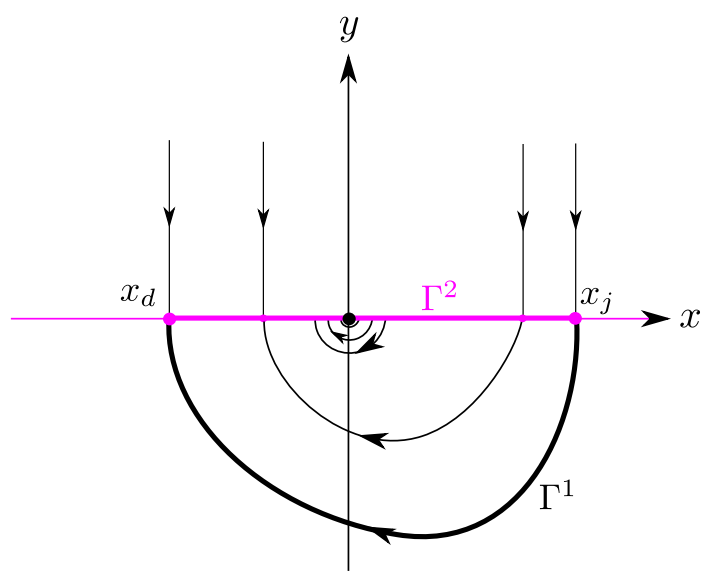

In addition we are able to identify the following asymptotics of a corresponding locally invariant (slow) manifold:

Lemma 1.4 Let $I \subset\left(-\infty, x_{j}\right)$, with $x_{j}$ as in (1.10), be a closed interval. Then for (1.1), there exists an exponentially attracting locally invariant (slow) manifold given as a graph:

$$
y=\epsilon h(x, \epsilon), x \in I, \epsilon \in\left[0, \epsilon_{0}\right),
$$

with $h$ smooth in both variables and $0<\epsilon_{0} \ll 1$.

The existence of locally invariant slow manifolds as described in Lemma 1.4 explains the observed quiescent (i.e. inactive) phase of the oscillations, see again Figs. 1 and 2. These slow manifolds are obtained as Fenichel slow manifolds [9] within the switching layer $y=\mathcal{O}(\epsilon)$. As such, the usual local invariance and contractivity properties apply. The term 'exponentially attracting' used above refers to the fact that each slow manifold is a base manifold for a stable asymptotic rate foliation with contraction along stable fibers greater than $e^{-c / \epsilon}$ for some constant $c>0$.

\subsubsection{The Le Corbeiller System}

As in the 'Hester case', due to the exponential term $e^{y \epsilon^{-1}}$, system (1.2) does not have a limit as $\epsilon \rightarrow 0$ for $y>0$. Again, we introduce a time transformation

$$
d t_{1}=\left(1+e^{y \epsilon^{-1}}\right) d t
$$

corresponding to multiplication of the vector-field by the strictly positive function $(1+$ $\left.e^{y \epsilon^{-1}}\right)^{-1}$, to obtain

$$
\begin{aligned}
& \dot{x}=\frac{y+a}{1+e^{y \epsilon^{-1}}}, \\
& \dot{y}=\frac{-x+2 b y}{1+e^{y \epsilon^{-1}}}-\frac{b y e^{y \epsilon^{-1}}}{1+e^{y \epsilon^{-1}}} .
\end{aligned}
$$


Fig. 6 Phaseportrait of the piecewise smooth system (1.13), (1.14) in black. The switching manifold $\Sigma=\{y=0\}$ is shown in magenta. Under the conditions (1.16), the singular cycle $\Gamma_{0}=\Gamma^{1} \cup \Gamma^{2}$ perturbs to a stable limit cycle by Theorem 1.5 (Color figure online)

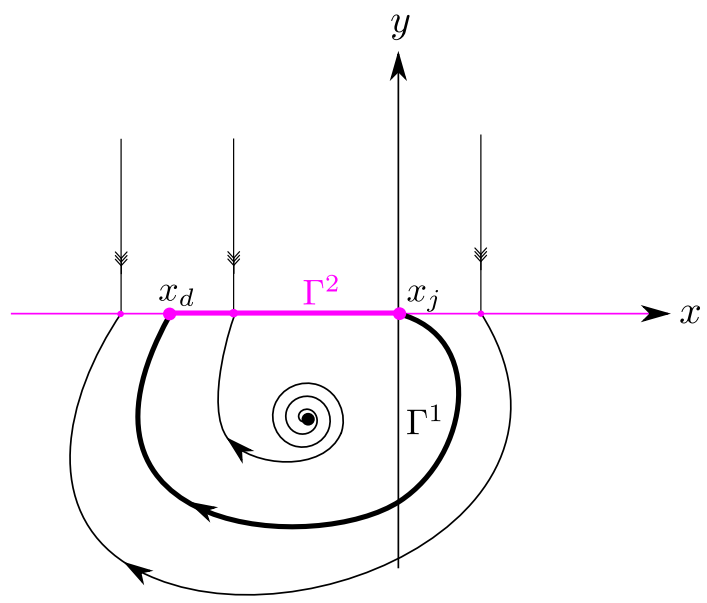

For this system, the pointwise limit as $\epsilon \rightarrow 0$ is well-defined for all $y \neq 0$, and gives the following PWS system:

$$
\begin{aligned}
& \dot{x}=0, \\
& \dot{y}=-b y,
\end{aligned}
$$

for $y>0$ and

$$
\begin{aligned}
& \dot{x}=y+a, \\
& \dot{y}=-x+2 b y,
\end{aligned}
$$

for $y<0$, with $\{y=0\}$ as switching manifold $\Sigma$. Some orbits of this limiting PWS system are shown in Fig. 6.

On the one hand, the $y<0$ system (1.14) has an unstable focus for $b \in(0,1)$ at $(x, y)=-a(2 b, 1)$, but also a quadratic, visible fold tangency [3] on the switching manifold at $(x, y)=(0,0)$. On the other hand, the $y>0$ system (1.13) has a line of equilibria along $\Sigma$. The Filippov system is therefore again completely degenerate along $\Sigma$.

Our analysis of the Le Corbeiller problem will reveal a reduced flow on an invariant manifold near the switching manifold $\Sigma$ for all $0<\epsilon \ll 1$ (see Lemma 1.6). Anticipating this, we define a singular relaxation cycle

$$
\Gamma_{0}=\Gamma^{1} \cup \Gamma^{2},
$$

where $\Gamma^{1}$ is the orbit segment of (1.14) obtained by flowing the tangency point $(0,0)$ forward until the first return $\left(x_{d}, 0\right)$, with $x_{d}<0$, to $\Sigma$, see Fig. 6 . The set $\Gamma^{2}$ is defined as the segment $\left(x_{d}, 0\right)$ on the switching manifold $\Sigma$. Notice that in contrast to the Hester system, there is no boundary focus point on $\Sigma$. Rather, the visible fold singularity [22] in the Le Corbeiller system provides a natural candidate for a concatenation point between cycle segments which can be identified on the PWS level, i.e. without the need to look in rescaled coordinates near $\Sigma$.

Our main result on the Le Corbeiller system is:

Theorem 1.5 Consider the Le Corbeiller system (1.2) and fix a large ball $B_{r}$ of radius $r$. Suppose that

$$
b \in(0,1), a>0 .
$$


Then there exists an $\epsilon_{0}>0$ such that for all $\epsilon \in\left(0, \epsilon_{0}\right)$, system (1.2) has a unique limit cycle $\Gamma_{\epsilon}$ in $B_{r}$. Furthermore, $\Gamma_{\epsilon}$ is attracting and

$$
\Gamma_{\epsilon} \rightarrow \Gamma_{0},
$$

as $\epsilon \rightarrow 0^{+}$, in Hausdorff-distance.

Again, we are also able to identify the following asymptotics of a corresponding locally invariant manifold with our methods:

Lemma 1.6 For system (1.2), fix a closed interval $I \subset(-\infty, 0)$. Then there exists an exponentially attracting locally invariant manifold given as a graph:

$$
y=\epsilon W(-x /(\epsilon b))\left(1-\epsilon b x^{-1} h\left(x, W(-x /(\epsilon b))^{-1}\right)\right),
$$

for $x \in I, \epsilon \in\left(0, \epsilon_{0}\right)$, with another smooth function $h$ satisfying $h(x, 0)=2$, and where $W:\left(-e^{-1}, \infty\right) \rightarrow(-1, \infty)$ is the principal Lambert-W function, defined by $z=W\left(z e^{z}\right)$ for all $z \in(-1, \infty)$.

Using the asymptotics

$$
W(w)=\log w\left(1+\mathcal{O}\left(\log ^{-1} w \log \log w\right)\right),
$$

of $W$ for $w \rightarrow \infty$, we realise that the invariant manifold (1.17) has the following leading order asymptotics

$$
y \sim \epsilon \log (-x /(\epsilon b))
$$

as $\epsilon \rightarrow 0^{+}$.

Remark 1.7 The two oscillations in Figs. 2 and 4, and the singular versions in Figs. 5 and 6, look qualitatively similar. Also the statements in Theorems 1.3 and 1.5 are almost identical. However, their PWS versions have different degeneracies along $\Sigma$ and we shall see that the systems are very different in the singular limit $\epsilon \rightarrow 0$, requiring different techniques for their analysis. For the Hester system (1.1) under the assumption (1.11) the exponentials do in fact not cause significant complications. In this respect the Le Corbeiller system (1.2) behaves very differently - the exponential terms lead to several complications which require a much more involved analysis. This is also reflected in the different asymptotics of their invariant manifolds presented in Lemma 1.4 and Lemma 1.6.

\subsection{Overview}

In Sect. 2, we first study (1.1) and prove Theorem 1.3. The proof, based upon the blow-up method and GSPT, is fairly straightforward, in particular in comparison with the proof of Theorem 1.5, which makes up the rest of the paper, see Sect. 3. Obviously, an essential step in the proof of Theorem 1.5 will be to prove Lemma 1.6. This is done in Sect. 3.2 after having described our blow-up approach. Subsequently, we present two lemmas Lemma 3.9 and Lemma 3.10 that prove Theorem 1.5, see Sects. 3.4 and 3.5. In Sect. 4 we then prove Lemma 3.9 before proving Lemma 3.10 in Sect. 5. Further details of the blow-up used to prove Lemma 3.10 are delayed to Sect. 6. In Sect. 7, we conclude our paper by presenting an outlook.

Remark 1.8 Throughout this paper, we will assume some familiarity with geometric singular perturbation theory and in particular with the blow-up method. The interested reader is referred to, e.g., $[23,25,27]$ for background and more references. 


\section{Blow-Up Analysis of the Hester System}

\subsection{The Scaling Approach}

Important insight into the dynamics of the Hester model (1.1) for small values of $\epsilon$ can be gained by first rescaling $y$ according to

$$
y=\epsilon y_{2},
$$

which provides a zoom into the dynamics close to the switching manifold $\Sigma$. This gives

$$
\begin{aligned}
\dot{x} & =\epsilon y_{2}, \\
\epsilon \dot{y}_{2} & =-x-2 \gamma \epsilon y_{2}+\mu\left(e^{y_{2}}-\kappa e^{(1+\alpha) y_{2}}\right) .
\end{aligned}
$$

System (2.2) is a standard slow-fast system, which has the form

$$
\begin{aligned}
& x^{\prime}=\epsilon^{2} y_{2}, \\
& y_{2}^{\prime}=-x-2 \gamma \epsilon y_{2}+\mu\left(e^{y_{2}}-\kappa e^{(1+\alpha) y_{2}}\right)
\end{aligned}
$$

on the fast time scale $\tau=t / \epsilon$. By setting $\epsilon=0$ we obtain the corresponding layer problem

$$
\begin{aligned}
& x^{\prime}=0, \\
& y_{2}^{\prime}=-x+\mu\left(e^{y_{2}}-\kappa e^{(1+\alpha) y_{2}}\right) .
\end{aligned}
$$

The set

$$
C=\left\{\left(x, y_{2}\right): x=\mu\left(e^{y_{2}}-\kappa e^{(1+\alpha) y_{2}}\right), y_{2} \in \mathbb{R}\right\},
$$

see Fig. 7, is a manifold of equilibria, which in GSPT is called the critical manifold of system (2.2). The critical manifold $C$ is the disjoint union of the following sets

$$
C_{a}=C \cap\left\{y_{2}>y_{j}\right\}, \quad F=C \cap\left\{y_{2}=y_{j}\right\}=\left\{\left(x_{j}, y_{j}\right)\right\}, \quad C_{r}=C \cap\left\{y_{2}<y_{j}\right\},
$$

recall (1.10), where

$$
y_{j}:=-\frac{1}{\alpha} \log (\kappa(1+\alpha))
$$

Here $C_{a}\left(C_{r}\right)$ is normally hyperbolic and attracting (repelling, respectively), whereas $F$ is a regular fold point. Since $C_{a}$ is normally hyperbolic, any compact submanifold perturbs to an attracting locally invariant slow manifold for $0<\epsilon \ll 1$ by Fenichel's theory [9]. This proves the statement regarding the invariant manifold in Lemma 1.4.

The reduced problem on $C$

$$
\begin{aligned}
& \dot{x}=y_{2}, \\
& 0=-x+\mu\left(e^{y_{2}}-\kappa e^{(1+\alpha) y_{2}}\right),
\end{aligned}
$$

is obtained by going to a (super) slow time and setting $\epsilon=0$. There is a unique equilibrium on $C$ at $y_{2}=0$. For $\kappa$ satisfying (1.11) the equilibrium is on the repelling branch $C_{r}$ and it is an unstable node. In this case the slow flow on $C_{a}$ is towards the right and approaches the fold point, where a fast downward jump along the unstable fiber of the layer problem occurs, see Fig. 7. 


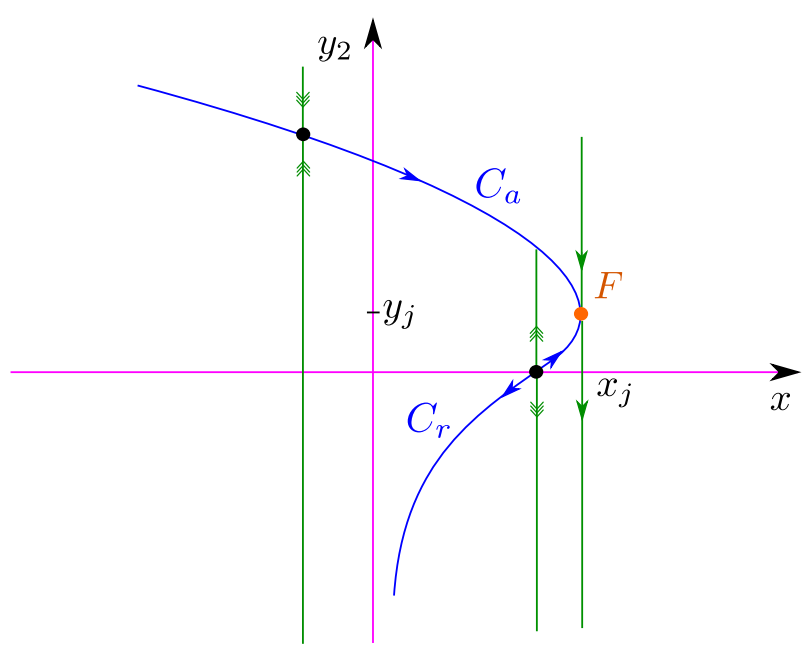

Fig. 7 Dynamics of system (2.2) in the singular limit $\epsilon=0$. Orbits of the layer problem are shown in green. The critical manifold $C$ and the reduced flow on it are shown in blue. The critical manifold $C$ has a fold point $F$ (orange), dividing $C$ into normally hyperbolic attracting and repelling branches $C_{a}$ and $C_{r}$ respectively. Under the conditions (1.11) there is an unstable node on the critical branch $C_{r}$ and the slow flow on $C_{a}$ has $\dot{x}>0$ (Color figure online)

Remark 2.1 The reduced flow on normally hyperbolic branches of $C$ occurs on a slow timescale for times that are $\mathcal{O}\left(\epsilon^{2}\right)$ slow with respect to the fast time $\tau$ in the switching layer, but only $\mathcal{O}(\epsilon)$ slow with respect to the original time $t$ in system (1.1). The fact that the timescale along $C$ is $\mathcal{O}(\epsilon)$ slow with respect to the original time $t$ explains (and is consistent with) the slow-fast relaxation oscillatory behaviour observed in Figs. 1 and 2.

Remark 2.2 In Fig. 7 and in all subsequent figures, we are following some conventions which are commonly used in GSPT to distinguish between the dynamics of the different limiting problems, which need to be shown simultaneously in the same figure: Green segments indicate "fast" orbits (of layer problems) whereas blue indicates slow flow (on critical manifolds) that is obtained upon (further) desingularization (by speeding up time). Orbits which approach equilibria (in forward or backward time) in hyperbolic directions are highlighted by tripleheaded arrows, whereas flows in slow and center directions are highlighted by single-headed arrows. Degenerate points, e.g. the fold point $F$, which need to be blown-up, are given individual colors, which are then also used for the corresponding blown up higher-dimensional objects.

Summing up, we have shown that the upper half of the limit cycle in the Hester problem can be obtained by the GSPT analysis of system (2.2): For $x<0$ there is fast dynamics towards $C_{a}$ along orbits of the layer problem, then there is slow flow along $C_{a}$ towards the fold point $F$, where a fast jump occurs with $y_{2}$ going to $-\infty$. The $x$-coordinate of the fold point $F$ is the value $x_{j}$ defining the right boundary of the segment $\Gamma^{2}$ of the singular cycle $\Gamma_{0}=\Gamma^{1} \cup \Gamma^{2}$, see Fig. 5 .

However, we have to keep in mind that solutions of system (2.2) with $y_{2}=\mathcal{O}(1)$ correspond to solutions of system (1.1) in a narrow strip $y=\mathcal{O}(\epsilon)$ around the switching surface $\Sigma$. To obtain the full limit cycle, we also have to consider the region $y=\mathcal{O}(1)<0$; this is not covered by the scaling (2.1). The analysis and the matching of these different regimes 
based on blow-up is carried out in the next subsection. Not surprisingly, we will see that the segment $\Gamma^{1}$ of the singular cycle $\Gamma_{0}$ is the orbit of the PWS system (1.9) starting at $\left(x_{j}, 0\right)$, see Fig. 5.

\subsection{The Blow-Up Approach}

To connect (2.2) with the PWS system (1.8) and (1.9), we apply a version of the blow-up method [6,25], see also [22-24].

As always in the blow-up approach one has to consider the extended system

$$
\begin{aligned}
x^{\prime} & =\epsilon \frac{y}{1+e^{(1+\alpha) y \epsilon^{-1}}}, \\
y^{\prime} & =\epsilon\left(\frac{-x-2 \gamma y+\mu e^{y \epsilon^{-1}}}{1+e^{(1+\alpha) y \epsilon^{-1}}}-\frac{\kappa \mu e^{(1+\alpha) y \epsilon^{-1}}}{1+e^{(1+\alpha) y \epsilon^{-1}}}\right), \\
\epsilon^{\prime} & =0,
\end{aligned}
$$

in $\mathbb{R}^{3}$ obtained from (1.7) written on the fast time defined by ()$\left.^{\prime}=\epsilon \dot{(}\right)$ by adding the trivial equation for $\epsilon$. This extended system has the $(x, y, 0)$-plane as a set of equilibria. The line $x \in \mathbb{R},(y, \epsilon)=(0,0)$ is singular in the sense of lack of smoothness of the vector field (2.5) as $\epsilon \rightarrow 0$. Recall that this degenerate line is precisely the switching manifold $\Sigma$ (embedded into $\mathbb{R}^{3}$ ) of the piecewise smooth system defined by (1.8) for $y>0$ and (1.9) for $y<0$. We regain smoothness by applying the blow-up transformation

$$
r \geq 0,(\bar{y}, \bar{\epsilon}) \in S^{1} \mapsto\left\{\begin{array}{l}
y=r \bar{y}, \\
\epsilon=r \bar{\epsilon},
\end{array}\right.
$$

blowing up the degenerate line to the cylinder $\mathbb{R} \times S^{1}$ with $r=0$, see Fig. 8. Since we are only interested in $\epsilon \geq 0$, only the part of the cylinder with $\bar{\epsilon} \geq 0$ is relevant. The edges $\bar{y}= \pm 1, \bar{\epsilon}=0, r=0$ of this half cylinder will be important later.

Remark 2.3 Note the color-coding which will be used frequently: the switching manifold $\Sigma$ shown in magenta in Fig. 5 is blown-up to the cylinder shown in magenta in Fig. 8.

The vector field (2.5) induces a vector field on the blown-up space. As always, the cylinder, corresponding to $r=0$, and the plane $\bar{\epsilon}=0$ are invariant and capture the crucial dynamics, both corresponding to $\epsilon=0$. Notice, that the scaling (2.1) can be viewed as a directional chart (obtained by setting $\bar{\epsilon}=1$ ) of the blow-up transformation, which covers the side of the cylinder corresponding to $\bar{\epsilon}>0$. In contrast to the usual blow-up approach [6,25], we will not divide by $r$. Thus, we find that the slow-fast system (2.3) multiplied by the positive and smooth function

$$
\frac{1}{1+e^{(1+\alpha) y_{2}}},
$$

which does not change the orbits, describes the blown-up dynamics in the chart corresponding to $\bar{\epsilon}=1$. In particular, on the cylinder we recover the limiting dynamics shown in Fig. 7.

In addition blow-up provides a compactification of system (2.2) as $y_{2} \rightarrow \pm \infty$. Thus the unstable fiber of the fold point $F$ limits now on a point on the edge $\bar{y}=-1, \bar{\epsilon}=0$, see Fig. 8 . Actually, the two edges $\bar{y}= \pm 1, \bar{\epsilon}=0, r=0$ of the half cylinder are lines of equilibria of the blown-up system, which must be studied in directional charts corresponding to $\bar{y}= \pm 1$. 


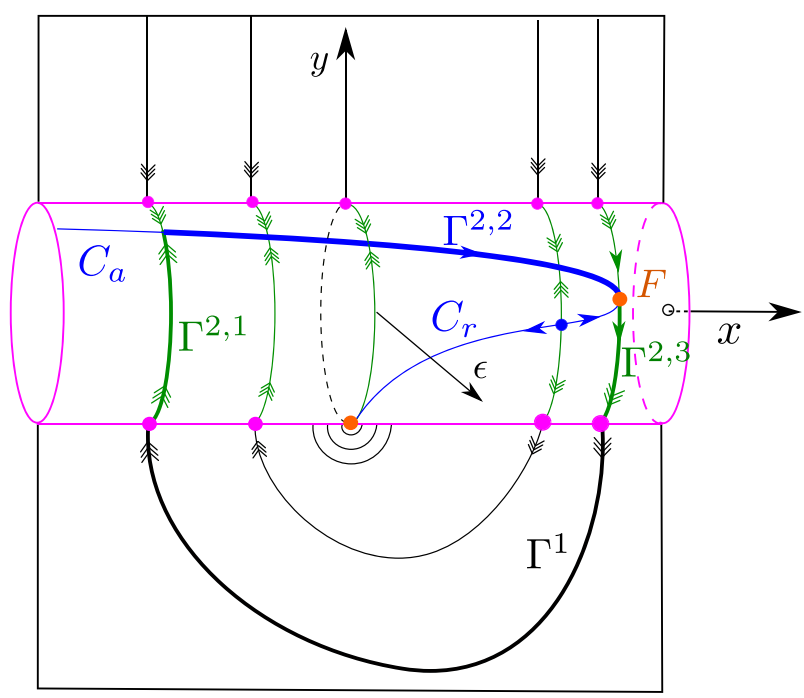

Fig. 8 Dynamics of system (2.5) after blow-up on the cylinder (magenta), corresponding to $r=0$, and in the plane (black) $\bar{\epsilon}=0$. We show a view from the top, i.e. from $\bar{\epsilon}>0$, with the orientation of the $x, y, \epsilon$ axis being indicated by black arrows labeled with $x, y, \epsilon$. The edges $\bar{y}= \pm 1, \bar{\epsilon}=0, r=0$ of the half cylinder are lines of equilibria, which are hyperbolic except at the point (orange) with $\bar{y}=-1, x=0$. In the plane $\bar{\epsilon}=0$ we recover the PWS dynamics (black) of system (1.8), (1.9). On the cylinder we recover the layer problem (green) and the reduced problem (blue) of system (2.2). This allows to define an improved singular cycle $\Gamma_{0}=\Gamma^{1} \cup \Gamma^{2,1} \cup \Gamma^{2,2} \cup \Gamma^{2,3}$ which perturbs to a true cycle $\Gamma_{\epsilon}$ for $\epsilon \ll 1$ (Color figure online)

In these charts we recover the PWS system (with improved hyperbolicity properties) within $\bar{\epsilon}=0$ after dividing out factors of $\bar{\epsilon}$, respectively. We illustrate our findings in Fig. 8.

The required analysis — to establish this rigorously—is standard, see e.g. [18,22-24]. Also, very similar computations are carried out in detail for the (more complicated) Le Corbeiller system below. We therefore only summarise the results for the Hester system. Along the edges $\bar{y}= \pm 1, \bar{\epsilon}=0$ we find lines of equilibria (magenta in Fig. 8) having a hyperbolic saddlestructure, except for $x=0, \bar{y}=-1, \bar{\epsilon}=0$ (orange circle) which is fully non-hyperbolic. This structure provides an improved singular cycle

$$
\Gamma_{0}=\Gamma^{1} \cup \Gamma^{2,1} \cup \Gamma^{2,2} \cup \Gamma^{2,3},
$$

(thick closed curve in Fig. 8), having good hyperbolicity properties except at the fold $F$ of the critical manifold $C$ (blue). Therefore, it is easy to perturb this singular cycle into an actual limit cycle for $0<\epsilon \ll 1$ by first considering a return mapping to the section $\{y=-\delta\}$ near $x_{j}$, for example, and then applying e.g. [25] to the passage near fold $F$ (working in the scaled coordinates (2.1)) to show that the Poincaré map is a strong contraction. We leave out the details because they are standard. See again [18] for a related system where more details are provided.

\section{Blow-Up Analysis of the Le Corbeiller System}

In the limit $\epsilon \rightarrow 0$ the transformed Le Corbeiller system (1.12) limits on the PWS system (1.13), (1.14) which has the singular cycle $\Gamma_{0}=\Gamma^{1} \cup \Gamma^{2}$, see Fig. 6. 


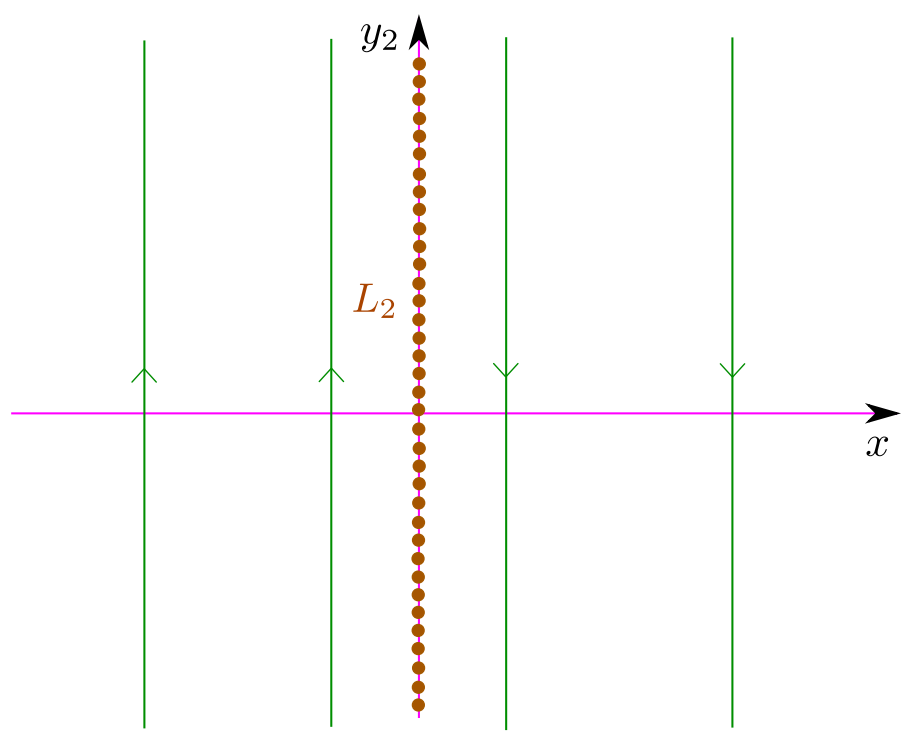

Fig. 9 Dynamics of system (3.2) in the singular limit $\epsilon=0$. Orbits of the layer problem (3.3) are shown in green, the flow going upwards for $x<0$ and downwards for $x>0$. The set $L_{2}$ defined by $x=0$ within $\epsilon=0$ is a line of degenerate singularities (Color figure online)

Motivated by the similarity of the two systems and by the success of the scaling approach for the Hester system, we begin our analysis by considering the original Le Corbeiller system (1.2) by rescaling

$$
y=\epsilon y_{2},
$$

i.e. by zooming into the switching manifold $\Sigma$. This produces the following system

$$
\begin{aligned}
\dot{x} & =\epsilon y_{2}+a, \\
\epsilon \dot{y}_{2} & =-x+b \epsilon y_{2}\left(2-e^{y_{2}}\right) .
\end{aligned}
$$

System (3.2) is a slow-fast system in standard form, with layer problem

$$
\begin{aligned}
& x^{\prime}=0, \\
& y_{2}^{\prime}=-x,
\end{aligned}
$$

which has a degenerate, non-hyperbolic line $L_{2}=\left\{\left(x, y_{2}\right): x=0\right\}$ of equilibria. Away from $x=0$ the flow is trivial and regular (upward for $x<0$, downward for $x>0$ ), see Fig. 9.

Remark 3.1 In contrast to the analysis of the Hester model based on the rescaling (2.1) in Sect. 2.1 the slow-fast dynamics of the the rescaled system (3.2) is quite degenerate, e.g. there exists no normally hyperbolic critical manifold. At this stage the rescaled system seems to capture very little of the observed limit cycle. Nevertheless, the flow defined by the rescaled system (3.2) and in particular the nonhyperbolic line $L_{2}$ of equilibria, will play an important role in a refined analysis of the limit cycle based on blow-up. More precisely, the flow of the rescaled system (3.2) will be recovered as the flow on the blow-up of the switching manifold $\Sigma$ to a cylinder, see Fig. 10. However, the full resolution of the Le Corbeiller model will require more than just one cylindrical blow-up due to its more singular dependence on $\epsilon$. 
To obtain a full resolution and to connect (3.2) with the PWS system (1.13) and (1.14) we study again the extended system

$$
\begin{aligned}
x^{\prime} & =\epsilon \frac{y+a}{1+e^{y \epsilon^{-1}},} \\
y^{\prime} & =\epsilon\left(\frac{-x+2 b y}{1+e^{y \epsilon^{-1}}}-\frac{b y e^{y \epsilon^{-1}}}{1+e^{y \epsilon^{-1}}}\right), \\
\epsilon^{\prime} & =0,
\end{aligned}
$$

obtained by transforming (1.12) to the fast time scale $\tau=t / \epsilon$ end adding the trivial equation for $\epsilon$.

The set defined by $(x, y, 0)$ is then a plane of equilibria, with the subset given by $y=0$ being extra singular due to the lack of smoothness there. We gain smoothness by applying the blow-up transformation

$$
r \geq 0,(\bar{y}, \bar{\epsilon}) \in S^{1} \mapsto\left\{\begin{array}{l}
y=r \bar{y}, \\
\epsilon=r \bar{\epsilon},
\end{array}\right.
$$

to the extended system. By this blow-up transformation the line $\{(x, 0,0), x \in \mathbb{R}\}$ corresponding to the switching manifold $\Sigma \times\{0\}$ is blown-up to a cylinder. Again only the part of the cylinder corresponding to $\bar{\epsilon} \geq 0$ is relevant for our analysis, see Fig. 10.

Three coordinate charts

$$
K_{1}: \bar{y}=-1, \quad K_{2}: \bar{\epsilon}=1, \quad K_{3}: \bar{y}=1,
$$

are necessary to analyze the dynamics on the blown-up space. We will make use of the usual subscript notation to specify coordinates in each chart, defining chart-specific coordinates as follows:

$$
\begin{array}{ll}
K_{1}: y=-r_{1}, & \epsilon=r_{1} \epsilon_{1}, \\
K_{2}: y=r_{2} y_{2}, & \epsilon=r_{2}, \\
K_{3}: y=r_{3}, & \epsilon=r_{3} \epsilon_{3} .
\end{array}
$$

Transition maps between a chart $K_{i}$ and $K_{j}(i \neq j)$ will be denoted by $\kappa_{i j}$, and are given here by

$$
\begin{array}{lll}
\kappa_{12}: r_{1}=-r_{2} y_{2}, & \epsilon_{1}=-y_{2}^{-1}, & y_{2}<0, \\
\kappa_{23}: y_{2}=\epsilon_{3}^{-1}, & r_{2}=r_{3} \epsilon_{3}, & \epsilon_{3}>0 .
\end{array}
$$

We will also adopt the convention of denoting a set $\gamma$ by $\gamma_{i}$ when viewed in a particular coordinate chart $K_{i}$.

Remark 3.2 Notice again that the scaling (3.1) corresponds to the directional chart $K_{2}$, which is hence customarily referred to as a "scaling chart". In the following we will call the chart $K_{1}$ an "entry chart", because in this chart the singular flow relevant for the limit cycle returns to the edge $(\bar{y}, \bar{\epsilon})=(-1,0)$ of the cylinder and continues from there on the cylinder, see see Fig. 10. Note that in charts $K_{i}, i=1,3$ the cylinder corresponds to $r_{i}=0$. These charts provide a compactification of the variable $y_{2}$ from the scaling chart $K_{2}$, i.e. $y_{2} \rightarrow-\infty$ in the plane $r_{2}=0$ corresponds to $\epsilon_{1} \rightarrow 0$ in the plane $r_{1}=0$, and $y_{2} \rightarrow \infty$ in the plane $r_{2}=0$ corresponds to $\epsilon_{3} \rightarrow 0$ in $r_{3}=0$, see (3.9).

Since it is the easiest chart to analyze we start with the scaling chart $K_{2}$. 


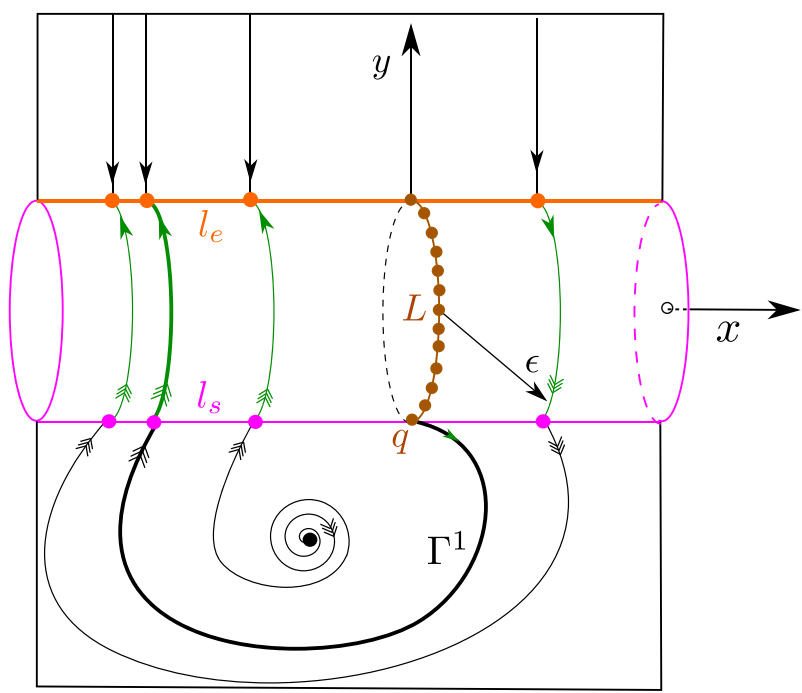

Fig. 10 Dynamics of system (3.4) after blow-up on the cylinder (magenta), corresponding to $r=0$, and in the plane (black) $\bar{\epsilon}=0$. We show a view from the top, i.e. from $\bar{\epsilon}>0$, with the orientation of the $x, y, \epsilon$ axis being indicated by black arrows labeled with $x, y, \epsilon$. Fast flow on the cylinder is shown in green. The line $l_{s}$ is a line of hyperbolic equilibria, except for the fully degenerate point $q \in l_{s} \cap L$ (brown). The line $l_{e}$ (orange) and the $L$ (brown) are lines of completely degenerate equilibria (Color figure online)

\section{Chart $K_{2}$}

The governing equations in chart $K_{2}$ are

$$
\begin{aligned}
x^{\prime} & =\frac{r_{2}\left(r_{2} y_{2}+a\right)}{1+e^{y_{2}}}, \\
y_{2}^{\prime} & =\frac{-x+r_{2} b y_{2}\left(2-e^{y_{2}}\right)}{1+e^{y_{2}}}, \\
r_{2}^{\prime} & =0,
\end{aligned}
$$

which is system (3.2) written on the fast time scale and multiplied by the positive and smooth factor

$$
\frac{1}{1+e^{y_{2}}} .
$$

Thus for $r_{2}=0$, i.e. on the cylinder, we recover the layer problem and in particular the fully nonhyperbolic line $L_{2}$ corresponding to $x=0$, see Fig. 10 .

\section{Chart $K_{1}$}

We now discuss the dynamics in the entry chart $K_{1}$. The governing equations are

$$
\begin{aligned}
& x^{\prime}=r_{1}\left(a-r_{1}\right), \\
& r_{1}^{\prime}=r_{1}\left(x+b r_{1}\left(2-e^{-\epsilon_{1}^{-1}}\right)\right), \\
& \epsilon_{1}^{\prime}=-\epsilon_{1}\left(x+b r_{1}\left(2-e^{-\epsilon_{1}^{-1}}\right)\right) .
\end{aligned}
$$


These equations are obtained by transforming to the new coordinates followed by a desingularization of the vector field corresponding to dividing out a factor of $\epsilon_{1}\left(1+e^{-\epsilon_{1}^{-1}}\right)^{-1}$, which is smooth for $\epsilon_{1} \geq 0$. Note, that it is this division that allows us to recover the PWS system within $\epsilon_{1}=0$, see Lemma 3.3. In particular, there is an isolated equilibrium at $p_{1}:=(-2 b a, a, 0)$, and two lines of equilibria

$$
l_{s, 1}=\{(x, 0,0): x \in \mathbb{R}\} \quad \text { and } \quad L_{1}=\left\{\left(0,0, \epsilon_{1}\right): \epsilon_{1} \geq 0\right\} .
$$

The following lemma summarizes important dynamical features of this system, which are illustrated in Fig. 10.

Lemma 3.3 The following hold for system (3.11):

(i) The linearization about any point $(x, 0,0)$ on the line of steady states $l_{s, 1}$ has eigenvalues

$$
\lambda=0, x,-x,
$$

and thus, the line $l_{s, 1}$ is a line of saddle points except at the origin $q_{1}:=(0,0,0)$ which is fully non-hyperbolic.

(ii) The line of steady states $L_{1}$ is fully non-hyperbolic, and coincides (where domains overlap) with the line $L_{2}$ observed in the $K_{2}$ chart.

(iii) The plane $\epsilon_{1}=0$ is invariant. Within this plane points $(x, 0,0) \in l_{s, 1}$ with $x>0$ are hyperbolic repelling, respectively hyperbolic attracting for $x<0$. The flow in this plane in $r_{1}>0$ is precisely the flow of the $y<0$ system (1.14) multiplied by a factor $r_{1}$, through the identification $y=-r_{1}$. The equilibrium $p_{1}=(-2 b a, a, 0)$ is an unstable focus within the invariant $\epsilon_{1}=0$ plane for any $b \in(0,1)$. Furthermore, there is a quadratic tangency between the flow of (3.11) $\left.\right|_{\epsilon_{1}=0}$ and $l_{s, 1}$ at $q_{1}$, corresponding to the tangency observed in Sect. 1.2.2. The equilibrium $p_{1}$ extends to $\epsilon_{1}>0$ as a line of equilibria which coincides upon coordinate change back to the original variables with the true equilibrium of the system identified in Sect. 1.2.2.

(iv) The plane $r_{1}=0$ is invariant. Within this plane points $\left(x_{b}, 0,0\right) \in l_{s, 1}$ with $x_{b}>0$ are hyperbolic attracting, respectively hyperbolic repelling for $x_{b}<0$, with stable, reps. unstable manifolds given by $x=x_{b}$.

Proof The statement (i) follows immediately from the linearization along $l_{s, 1}$, and the simple form of the equations when restricted to the invariant plane $r_{1}=0$ :

$$
\begin{aligned}
& x^{\prime}=0, \\
& \epsilon_{1}^{\prime}=-\epsilon_{1} x .
\end{aligned}
$$

The statement (ii) follows by an application of the transition map $\kappa_{12}$ given in (3.9), and the statements (iii) and (iv) follow by simple calculations made using the system governing the dynamics in the invariant plane $\epsilon_{1}=0$ :

$$
\begin{aligned}
x^{\prime} & =r_{1}\left(a-r_{1}\right), \\
r_{1}^{\prime} & =r_{1}\left(x+2 b r_{1}\right) .
\end{aligned}
$$




\section{Chart $K_{3}$}

Dynamics in chart $K_{3}$ are governed by

$$
\begin{aligned}
x^{\prime} & =r_{3} e^{-\epsilon_{3}^{-1}}\left(a+r_{3}\right), \\
r_{3}^{\prime} & =-r_{3}\left(b r_{3}+e^{-\epsilon_{3}^{-1}}\left(x-2 b r_{3}\right)\right), \\
\epsilon_{3}^{\prime} & =\epsilon_{3}\left(b r_{3}+e^{-\epsilon_{3}^{-1}}\left(x-2 b r_{3}\right)\right),
\end{aligned}
$$

after desingularization through division of the right hand side by $\epsilon_{3}\left(1+e^{-\epsilon_{3}^{-1}}\right)^{-1}$. In particular, there are two lines of equilibria:

$$
l_{e, 3}=\{(x, 0,0): x \in \mathbb{R}\} \quad \text { and } \quad L_{3}=\left\{\left(0,0, \epsilon_{3}\right): \epsilon_{3} \geq 0\right\} .
$$

Restricted to the invariant planes $r_{3}=0$ and $\epsilon_{3}=0$, we obtain the following equations

$$
\begin{aligned}
& x^{\prime}=0, \\
& \epsilon_{3}^{\prime}=\epsilon_{3} x e^{-\epsilon_{3}^{-1},}
\end{aligned}
$$

and

$$
\begin{aligned}
& x^{\prime}=0, \\
& r_{3}^{\prime}=-b r_{3}^{2},
\end{aligned}
$$

respectively. It is clear that any point in either $l_{e, 3}$ and $L_{3}$ is fully non-hyperbolic. We notice in particular the 'exponential loss of hyperbolicity' in (3.15) as $\epsilon_{3} \rightarrow 0$. The following lemma summarizes the important features of this system, which are illustrated in Fig. 10.

Lemma 3.4 The following hold for system (3.14):

(i) The line of steady states $l_{e, 3}$ is fully non-hyperbolic.

(ii) The line of steady states $L_{3}$ is fully non-hyperbolic, and coincides where domains overlap with the non-hyperbolic line $L_{2}$ observed in the $K_{2}$ chart.

(iii) The plane $\epsilon_{3}=0$ is invariant. The flow in this plane in $r_{3}>0$ is precisely the $y>0$ system (1.13) multiplied by a factor $r_{3}$, through the identification $y=r_{3}$. The flow in $\epsilon_{3}=0, r_{3}>0$ is parallel to the $r_{3}$-axis and toward $l_{e, 3}$.

(iv) The plane $r_{3}=0$ is invariant. The flow in this plane for $\epsilon_{3}>0$ is parallel to the $x$-axis, and toward (away from) the line $l_{e, 3}$ for $x<0(x>0)$.

Proof Straightforward.

We briefly sum up the results obtained by the above analysis, which are illustrated in in Fig. 10. We have achieved a certain desingularization of (3.4) by means of the blow-up transformation (3.5). On the cylinder $r=0$ we have recovered the layer problem of (3.2) in particular the nonhyperbolic line $L$. In the invariant plane $\bar{\epsilon}=0$ we have recovered the PWS system (1.14) and (1.13) for $\bar{y}<0$ respectively $\bar{y}>0$. At the invariant line $l_{s}$ of saddle type we have gained hyperbolicity away from the nonhyperbolic point $q$. The invariant lines $l_{e}$ and $L$ are still fully degenerate and will be treated by further blow-ups. 


\subsection{Blow-Up in the Exponential Regime}

To deal with the (exponential) loss of normal hyperbolicity associated with the line $l_{e, 3}$ in system (3.15), we use the approach put forward in [20]: introduce the auxiliary variable

$$
q=e^{-\epsilon_{3}^{-1}}
$$

and extend the phase space by including an equation for $q^{\prime}$. For improved readability (in subsequent blow-up transformations) we drop the subscripts in the coordinates of chart $K_{3}$ in the following. Thus, the following system

$$
\begin{aligned}
x^{\prime} & =r \epsilon q(a+r), \\
r^{\prime} & =-r \epsilon(b r+q(x-2 b r)), \\
\epsilon^{\prime} & =\epsilon^{2}(b r+q(x-2 b r)), \\
q^{\prime} & =q(b r+q(x-2 b r)),
\end{aligned}
$$

is obtained after a further multiplication of the right hand side by $\epsilon$; we therefore basically undo the division by $\epsilon$ used to obtain (3.14). It is worth keeping in mind that

$$
Q=\left\{(x, r, \epsilon, q): q=e^{-\epsilon^{-1}}\right\}
$$

is invariant. We will often use this fact, utilizing it when it helps in the analysis.

In system (3.16), the non-hyperbolic line of equilibria $L_{3}$ in (3.14) shows up as the $Q$ restricted subset:

$$
L_{e}=\mathcal{P} \cap Q=\left\{\left(0,0, \epsilon, e^{-\epsilon^{-1}}\right): \epsilon \geq 0\right\},
$$

of the non-hyperbolic plane of equilibria

$$
\mathcal{P}=\{(0,0, \epsilon, q): \epsilon, q \geq 0\} .
$$

The subscript ' $e$ ' (for 'exponential') in $L_{e}$ signifies that we are considering the object in the extended, four-dimensional system (3.16). Similarly, the line of equilibria

$$
\mathfrak{l}_{e}=\{(x, 0,0,0): x \in \mathbb{R}\},
$$

can be viewed as an improved version of the line of degenerate equilibria $l_{e, 3}$. It is the intersection of two separate degenerate planes: $\{(x, 0,0, q): x \in \mathbb{R}, q \geq 0\}$ and

$$
\mathcal{P}_{e}=\{(x, 0, \epsilon, 0): x \in \mathbb{R}, \epsilon \geq 0\} .
$$

Although the extended system (3.16) is clearly quite degenerate, the main advantage of this system is that the loss of normal hyperbolicity is now algebraic, and blow-up methods are applicable. Therefore, we introduce a blow-up of the plane of equilibria $\mathcal{P}_{e}$ of system (3.16) via the map

$$
\rho \geq 0,(\bar{r}, \bar{q}) \in S^{1} \mapsto\left\{\begin{array}{l}
r=\rho \bar{r}, \\
q=\rho \bar{q} .
\end{array}\right.
$$

We are primarily interested in the dynamics observable in the entry chart

$$
\mathfrak{K}_{1}: \bar{q}=1,
$$


which has chart specific coordinates

$$
r=\rho_{1} r_{1}, \quad q=\rho_{1} .
$$

In this chart, we obtain the system

$$
\begin{aligned}
x^{\prime} & =\rho_{1} r_{1} \epsilon\left(a+\rho_{1} r_{1}\right), \\
r_{1}^{\prime} & =-r_{1}(1+\epsilon)\left(x+b r_{1}\left(1-2 \rho_{1}\right)\right), \\
\epsilon^{\prime} & =\epsilon^{2}\left(x+b r_{1}\left(1-2 \rho_{1}\right)\right), \\
\rho_{1}^{\prime} & =\rho_{1}\left(x+b r_{1}\left(1-2 \rho_{1}\right)\right),
\end{aligned}
$$

after a suitable desingularization (division by $\rho_{1}$ ). Note that the set

$$
Q_{1}=\left\{\left(x, r_{1}, \epsilon, \rho_{1}\right): \rho_{1}=e^{-\epsilon^{-1}}\right\}
$$

is invariant under the flow induced by (3.22). The non-hyperbolic plane $\mathcal{P}$ defined in (3.18) becomes a plane of equilibria for system (3.22),

$$
\mathcal{P}_{1}=\left\{\left(0,0, \epsilon, \rho_{1}\right): \epsilon \geq 0, \rho_{1} \geq 0\right\},
$$

and

$$
\mathfrak{l}_{e, 1}=\{(x, 0,0,0): x \in \mathbb{R}\}
$$

constitutes a line of equilibria for the system (3.22). We also have

$$
L_{e, 1}=\mathcal{P}_{1} \cap Q_{1}=\left\{\left(0,0, \epsilon, e^{-\epsilon^{-1}}\right): \epsilon \geq 0\right\},
$$

which will be important for our analysis.

Lemma 3.5 The following hold for the system (3.22):

(i) Within $\epsilon=0$, there exists a two-dimensional manifold of equilibria given by

$$
C_{1}=\left\{\left(x,-\frac{x}{b\left(1-2 \rho_{1}\right)}, 0, \rho_{1}\right): x \leq 0, \rho_{1} \in\left[0, \beta_{1}\right]\right\} .
$$

The linearization about any point in $C_{1}$ has a single non-trivial eigenvalue

$$
\lambda=\frac{x}{b\left(1-2 \rho_{1}\right)} \leq 0,
$$

where $\beta_{1}>0$ is chosen sufficiently small for the inequality to hold. Accordingly, $C_{1}$ is normally hyperbolic and attracting within $\epsilon=0$ for $x<0$, and non-hyperbolic along the line $\mathfrak{I}^{1}=\left\{\left(0,0,0, \rho_{1}\right): \rho_{1} \in\left[0, \beta_{1}\right]\right\}$.

(ii) Within $\rho_{1}=0$, there exists a two-dimensional manifold of equilibria given by

$$
S_{1}=\left\{\left(x,-\frac{x}{b}, \epsilon, 0\right): x \leq 0, \epsilon \in\left[0, \beta_{2}\right]\right\} .
$$

The linearization about any point in $S_{1}$ has a single non-trivial eigenvalue

$$
\lambda=\frac{x}{b} \leq 0
$$

Accordingly, $S_{1}$ is normally hyperbolic and attracting within within $\rho_{1}=0$ for $x<0$, and non-hyperbolic along the line $\mathfrak{I}^{2}=\left\{(0,0, \epsilon, 0): \epsilon \in\left[0, \beta_{2}\right]\right\}$. Note that $\mathfrak{I}^{1} \cap \mathfrak{I}^{2}=$ $\{(0,0,0,0)\}$. 
(iii) The manifolds $C_{1}$ and $S_{1}$ intersect the invariant domain of interest $Q_{1}$ along a onedimensional manifold of equilibria $\mathcal{C}_{1}$ satisfying

$$
\mathcal{C}_{1}=C_{1} \cap Q_{1}=S_{1} \cap Q_{1}=C_{1} \cap S_{1}=\left\{\left(x,-\frac{x}{b}, 0,0\right): x \leq 0\right\} .
$$

Considered within the invariant plane $\epsilon=\rho_{1}=0$, the manifold $\mathcal{C}_{1}$ is normally hyperbolic and attracting for $x<0$, being non-hyperbolic at the point $P:(0,0,0,0)$.

(iv) The plane $\mathcal{P}_{1}$ is fully non-hyperbolic, and $L_{e, 1}$ coincides with the image of the nonhyperbolic line $L_{3}$ observed in system (3.14) (recall Lemma 3.4).

(v) The eigenvalues associated with the linearization along $\mathfrak{l}_{e, 1}$ are given by

$$
\lambda=0,-x, 0, x,
$$

implying that $\mathfrak{l}_{e, 1}$ is a line of partially hyperbolic saddles for $x \neq 0$, and non-hyperbolic for $x=0$.

Proof The statement (i) follows after linearization of the system obtained by restricting to the invariant hyperplane $\epsilon=0$ :

$$
\begin{aligned}
& x^{\prime}=0, \\
& r_{1}^{\prime}=-r_{1}\left(x+b r_{1}\left(1-2 \rho_{1}\right)\right), \\
& \rho_{1}^{\prime}=\rho_{1}\left(x+b r_{1}\left(1-2 \rho_{1}\right)\right) .
\end{aligned}
$$

Similarly, the statement (ii) is obtained after linearization of the system obtained by restricting to the invariant hyperplane $\rho_{1}=0$ :

$$
\begin{aligned}
& x^{\prime}=0, \\
& r_{1}^{\prime}=-r_{1}(1+\epsilon)\left(x+b r_{1}\right), \\
& \epsilon^{\prime}=\epsilon^{2}\left(x+b r_{1}\right) .
\end{aligned}
$$

Statement (iii) follows immediately from (i)-(ii) and the observation that $\rho_{1}=e^{-\epsilon^{-1}}=$ $0 \Longrightarrow \rho_{1}=\epsilon=0$.

Statement (iv) follows after linearization of the system (3.22), together with an application of the blow-down transformation.

Statement (v) also follows after linearization of the system (3.22).

The most important properties of system (3.22) described in Lemma 3.5 are sketched in Fig. 11.

Remark 3.6 Clearly, not all properties of the four-dimensional system (3.22) are visible in Fig. 11, which focuses on explaining how the second blow-up improves the situation shown in Fig. 10. Keep in mind, that the second blow-up (3.20) affects only a small neighborhood of the line $l_{e}$ in Fig. 10. The degenerate line $l_{e}$ is blown up to the orange cylinder, corresponding to $\rho=0$, i.e. $\rho_{1}=0$ in chart (3.21). In this chart $r_{1}>0$ means "going up" on the cylinder, which due to $y=r_{3}=\rho_{1} r_{1}$ justifies our use of the $y$-axis in the figure.

The preceding observations are sufficient to prove the following result, which will in turn allow for a proof of Lemma 1.6.

Lemma 3.7 Fix a closed interval $I \subset(-\infty, 0)$. The system (3.22) then has a threedimensional locally invariant manifold $M_{1}$ taking the form of a graph

$$
r_{1}=-\frac{x}{b\left(1-2 \rho_{1}\right)}+\epsilon \rho_{1} m\left(x, \epsilon, \rho_{1}\right), \quad x \in I, \rho_{1} \in\left[0, \beta_{1}\right], \epsilon \in\left[0, \beta_{2}\right],
$$




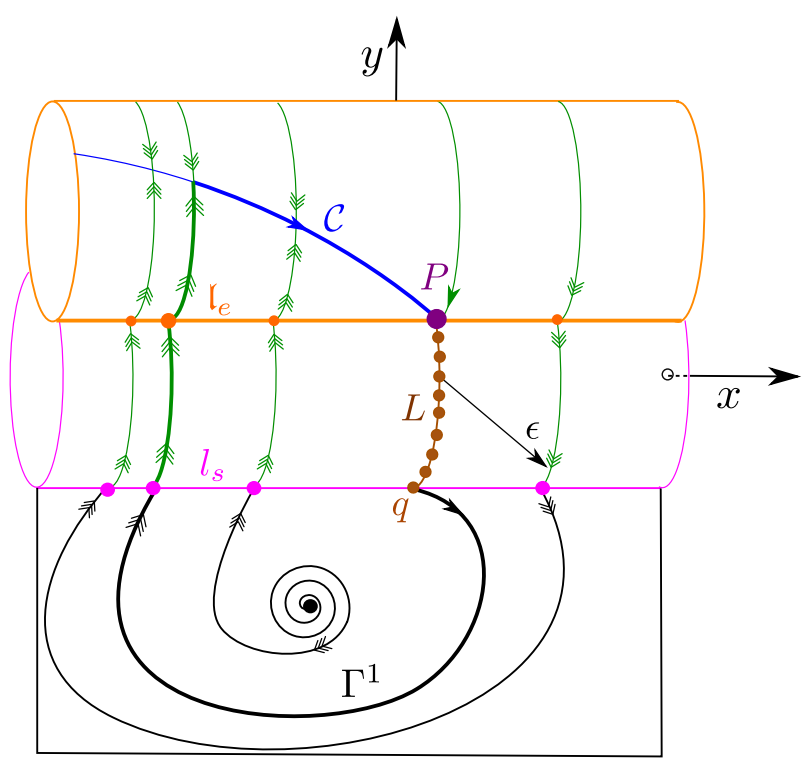

Fig. 11 Dynamics upon blow-up (3.20) of $l_{e}$ on the cylinder (orange), corresponding to $\rho=0$. We show a view from the top, i.e. from $\bar{\epsilon}>0$, with the orientation of the $x, y, \epsilon$ axis being indicated by black arrows labeled with $x, y, \epsilon$. Fast flow on the orange cylinder is shown in green. In comparison with Fig. 10 we have gained hyperbolicity at the line of equilibria $\mathfrak{l}_{e}$ and we are able to identify a normally hyperbolic critical manifold $\mathcal{C}$ (blue). The line (half-circle) $L$ (brown) of equilibria and, in particular, the equilibrium $P$ (purple) are still degenerate and will require additional blow-up transformations (Color figure online)

for $\beta_{i}>0 i=1,2$ sufficiently small, and where $m\left(x, \epsilon, \rho_{1}\right)$ is smooth. The manifold $M_{1}$ contains the manifolds of equilibria $C_{1}$ and $S_{1}$ within the invariant hyperplanes $\epsilon=0$ and $\rho_{1}=0$, respectively, and the dynamics restricted to $M_{1}$ has $\dot{x}=$ a to leading order in the $x$-direction, with respect to the original time in (1.2).

Proof Follows from center manifold theory and the statements in Lemma 3.5.

\subsection{Proof of Lemma 1.6}

To prove Lemma 1.6 and the expression in (1.17) we simply restrict $M_{1}$ in (3.26) to the invariant set $Q_{1}$ and blow down to the ( $\left.x, y\right)$-coordinates. Using (3.8), (3.21) and by returning the subscript 3 on $\epsilon$ in (3.26), we obtain the following equations

$$
y=e^{-\epsilon_{3}^{-1}}\left(-\frac{x}{b}\right)\left(1+e^{-\epsilon_{3}^{-1}} \tilde{m}\left(x, \epsilon_{3}, e^{-\epsilon_{3}^{-1}}\right)\right),
$$

and

$$
\epsilon=e^{-\epsilon_{3}^{-1}}\left(-\frac{x}{b}\right)\left(1+e^{-\epsilon_{3}^{-1}} \tilde{m}\left(x, \epsilon_{3}, e^{-\epsilon_{3}^{-1}}\right)\right) \epsilon_{3},
$$

where $0<\epsilon \ll 1$ is the original small parameter. In these expressions we have also introduced a new $\tilde{m}$ obtained by expanding the first term in (3.26) about $\rho_{1}=0$ and setting $-x / b>0$ outside a bracket. It is straightforward to show that $\tilde{m}(x, 0,0)=2$. The expression in (1.17) is the result of solving these equations for $y$ as a function of $\epsilon$. Let

$$
Z(s)=W\left(s^{-1}\right)^{-1},
$$


for any $s>0$, where $W$ is the Lambert-W function satisfying $W(t) e^{W(t)}=t$ for every $t>0$. Then

$$
s=Z(s) e^{-Z(s)^{-1}},
$$

and $Z$ has a continuous extension to $s=0$ with value $Z(0)=0$. We then solve (3.28) by introducing the auxiliary variable $u$ through the expression

$$
\epsilon_{3}=Z(\epsilon b /(-x))(1+Z(\epsilon b /(-x)) u)^{-1} .
$$

Notice that

- $u=0$ in this expression by (3.30) gives the 'leading order solution' of (3.28) obtained by setting $\tilde{m} \equiv 0$.

- Once we have obtained $\epsilon_{3}$ as a function of $\epsilon$, we obtain the desired $y$ as a function of $\epsilon$ from (3.27) as

$$
y=\epsilon \epsilon_{3}^{-1} \text {. }
$$

Write

$$
z:=Z(\epsilon b /(-x))
$$

Then inserting (3.31) into (3.28) produces the following equation

$$
1=e^{-u}(1+z u)^{-1}\left(1+e^{-z^{-1}} e^{-u} \tilde{m}\left(x, z(1+z u)^{-1}, e^{-z^{-1}} e^{-u}\right)\right),
$$

after canceling out a common factor on both sides obtained from (3.30) with $s=\epsilon b /(-x)$. This equation is smooth in $u, x$ and $z \geq 0$. In particular, $u=z=0$ is a solution for any $x \in I$ and the partial derivative of the right hand side with respect to $u$ gives -1 for $u=z=0$, $x \in I$. Therefore, by applying the implicit function theorem, we obtain a locally unique solution $u=\tilde{h}(x, z)$ of (3.34) with $\tilde{h}$ smooth, satisfying $\tilde{h}(x, 0)=0$ for all $x \in I$, and where $z \in\left[0, \beta_{3}\right]$ for $\beta_{3}>0$ sufficiently small. A simple computation shows that $\tilde{h}$ is exponentially small with respect to $z$ :

$$
\tilde{h}(x, z)=e^{-z^{-1}} h(x, z),
$$

for some smooth $h$ satisfying $h(x, 0)=\tilde{m}(x, 0,0)=2$. Inserting (3.33) into this expression produces, by (3.31) and (3.30), the following locally unique solution of (3.28)

$$
\epsilon_{3}=Z(\epsilon b /(-x))\left(1-\epsilon b x^{-1} h(x, Z(\epsilon b /(-x)))\right)^{-1} .
$$

Finally, by inserting this into (3.32) we obtain the expression

$$
y=\epsilon Z(\epsilon b /(-x))^{-1}\left(1-\epsilon b x^{-1} h(x, Z(\epsilon b /(-x)))\right),
$$

which by (3.29) gives (1.17).

\subsection{Improved Singular Cycle and Preliminary Results}

The analysis thus far is sufficient for the construction of an improved singular orbit and corresponding Poincaré map. The singular orbit can now be viewed as a union of distinct orbit segments $\Gamma^{i}, i=1,2,3,4$, and the line $L$. The segment $\Gamma^{1}$ is already defined as the part of the singular cycle contained in the half plane $y<0$, and $x_{d}<0$ is the $x$-coordinate of the first intersection of the trajectory flowed forward from $(0,0)$ with the line $y=0$ in system 


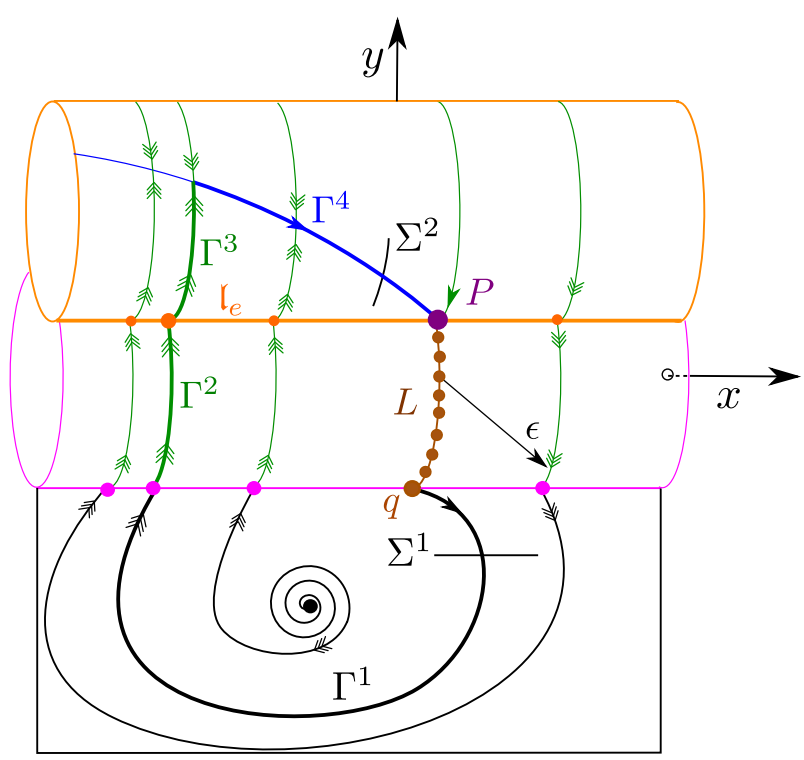

Fig. 12 Due to the improved hyperbolicity properties obtained by the second blow-up (3.20), we are able to identify a less degenerate singular cycle $\Gamma_{0}=\Gamma^{1} \cup \Gamma^{2} \cup \Gamma^{3} \cup \Gamma^{4} \cup L$. The sections $\Sigma^{1}$ and $\Sigma^{2}$ used to define the Poincaré mapping $\Pi=\Pi^{2} \circ \Pi^{1}$ are also shown

(1.14); see the discussion leading to the expression (1.15) in Sect. 1.2.2. The remaining segments $\Gamma^{i}$ are are listed below, together with the coordinate chart used to define them.

$$
\begin{cases}\Gamma^{2}=\left\{\left(x_{d}, y_{2}, 0\right): y_{2} \in \mathbb{R}\right\}, & \text { chart } K_{2}, \\ \Gamma^{3}=\left\{\left(x_{d}, r_{1}, 0,0\right): r_{1} \in\left[0,-b^{-1} x_{d}\right]\right\}, & \text { chart } \mathfrak{K}_{1}, \\ \Gamma^{4}=\left\{\left(x_{1},-b^{-1} x_{1}, 0,0\right): x_{1} \in\left[x_{d}, 0\right]\right\}, & \text { chart } \mathfrak{K}_{1}, \\ L=\left\{\left(0, y_{2}, 0\right): y_{2} \in \mathbb{R}\right\}, & \text { chart } K_{2} .\end{cases}
$$

Remark 3.8 Note that we are permitting a slight abuse of notation here by allowing $\Gamma^{2}$ to refer to a different segment as in Sect. 1.2.2, expression (1.15). In fact, it is $\Gamma^{2} \cup \Gamma^{3} \cup \Gamma^{4} \cup L$ in (3.35) that upon blowing down to the $(x, y)$-plane becomes $\Gamma^{2}$ in (1.15).

We can now define an improved singular cycle $\Gamma_{0}$ by

$$
\Gamma_{0}=\Gamma^{1} \cup \Gamma^{2} \cup \Gamma^{3} \cup \Gamma^{4} \cup L,
$$

see Fig. 12. Although this cycle has improved hyperbolicity properties, it is still completely degenerate along $L$. A fully nondegenerate singular cycle, resolving the dynamics around $L$, will be presented in Sect. 5.1, relying on details in Sect. 6 once the remaining prerequisite blow-ups have been defined. 


\subsection{The Poincaré map $\Pi=\Pi^{2} \circ \Pi^{1}$}

Also shown in Fig. 12 are two sections $\Sigma^{1}$ and $\Sigma^{2}$, both transversal to $\Gamma_{0}$. We define these as follows:

$$
\begin{cases}\Sigma^{1}=\left\{(x,-\delta, \epsilon): x \in[-\alpha, \alpha], \epsilon \in\left[0, \epsilon_{0}\right]\right\}, & (x, y, \epsilon), \\ \Sigma^{2}=\left\{\left(-\eta, r_{1}, \epsilon, \rho_{1}\right): r_{1} \in[0, R], \epsilon \in[0, \beta], \rho_{1} \in[0, \beta]\right\}, & \text { chart } \mathfrak{K}_{1},\end{cases}
$$

for suitably chosen, small positive constants $\delta, \alpha, \epsilon_{0}, \eta, R, \beta$. We then define the Poincaré map $\Pi: \Sigma^{1} \rightarrow \Sigma^{1}$ induced by the flow of the extended system $((1.12), \dot{\epsilon}=0)$ in terms of the composition

$$
\Pi=\Pi^{2} \circ \Pi^{1}: \Sigma^{1} \rightarrow \Sigma^{1},
$$

where $\Pi^{1}: \Sigma^{1} \rightarrow \Sigma^{2}$, and $\Pi^{2}: \Sigma^{2} \rightarrow \Sigma^{1}$ denote transition maps induced by the flow. To prove Theorem 1.5 we consider each of $\Pi^{1}$ and $\Pi^{2}$ in turn.

First, we consider the map $\Pi^{1}$ and write

$$
\Pi^{1}:(x,-\delta, \epsilon) \mapsto\left(-\eta, r_{1}^{1}(x, \epsilon), W\left(r_{1}^{1}(x, \epsilon) / \epsilon\right)^{-1}, \epsilon W\left(r_{1}^{1}(x, \epsilon) / \epsilon\right)\right) .
$$

To obtain the last two components of $\Pi^{1}$, we have used (3.8), (3.21) and the invariance of the set $Q$.

Lemma 3.9 The following holds for $\alpha>0$ and $\epsilon_{0}>0$ sufficiently small:

(i) $\Pi^{1}$ is well-defined and at least $C^{1}$ with respect to $x$.

(ii) The image $\Pi^{1}\left(\Sigma^{1}\right) \subset \Sigma^{2}$ is an exponentially thin wedge-shaped region about $M \cap \Sigma^{2}$, where $M$ is the center manifold in Lemma 3.7.

(iii) In particular, the restricted map $x \mapsto r_{1}^{1}(x, \epsilon)$ for any $\epsilon \in\left(0, \epsilon_{0}\right]$ is a strong contraction, i.e.

$$
\begin{aligned}
r_{1}^{1}(x, \epsilon) & =\frac{\eta}{b}+\mathcal{O}\left(\epsilon \log \epsilon^{-1}\right), \\
\frac{\partial}{\partial x} r_{1}^{1}(x, \epsilon) & =\mathcal{O}\left(e^{-c / \epsilon}\right),
\end{aligned}
$$

for some constant $c>0$.

Given the form of the map in (3.37) and Lemma 3.9, we write $\Pi^{2}$ as follows

$$
\Pi^{2}:\left(-\eta, r_{1}, W\left(r_{1} / \epsilon\right)^{-1}, \epsilon W\left(r_{1} / \epsilon\right)\right) \mapsto\left(x^{2}\left(r_{1}, \epsilon\right),-\delta, \epsilon\right),
$$

for any $r_{1} \in I, \epsilon \in[0, \delta]$. Here $I$ is a small neighborhood of $\eta / b$, recall (3.38). The form in (3.39) restricts $\Pi^{2}$ to the relevant subset of $\Sigma^{2}$ defined by invariance of $\epsilon$ and the set $Q$, and allows for composition with $\Pi^{1}$, see (3.37).

Lemma 3.10 The following hold for $R, \beta>0$ and $\epsilon_{0}$ sufficiently small:

(i) $\Pi^{2}$ is well-defined and at least $C^{1}$ with respect to $r_{1}$.

(ii) The restricted map $r_{1} \mapsto x^{2}\left(r_{1}, \epsilon\right)$ for any $\epsilon \in\left(0, \epsilon_{0}\right]$ is a strong contraction, i.e.

$$
\frac{\partial}{\partial r_{1}} x^{2}\left(r_{1}, \epsilon\right)=\mathcal{O}\left(e^{-c / \epsilon}\right) \text {, }
$$

for some constant $c>0$. 


\subsection{Proof of Theorem 1.5}

Taken together, Lemma 3.9 and Lemma 3.10 show that $\Pi$ is a contraction. Theorem 1.5 and the existence of a perturbed limit cycle $\Gamma_{\epsilon}$ for all $0<\epsilon \ll 1$ is then a consequence of the contraction mapping theorem. Lemma 3.9 is proved in Sect. 4. A rigorous proof of Lemma 3.10 requires additional blow-ups. We summarise these in in Sect. 5, see Sect. 5.1, allowing for the proof of Lemma 3.10 to be outlined in Sect. 5.2. The blow-up analysis outlined for the purposes of proving Lemma 3.10 are given in greater detail in Sect. 6 .

Remark 3.11 It follows from Lemma 3.9 and Lemma 3.10 that $\Pi$ is a strong contraction even though the both active and inactive phases of the corresponding relaxation oscillations occur on timescales of $\mathcal{O}(1)$ as $\epsilon \rightarrow 0$, see again Figs. 3 and 4, i.e. $\Pi$ is a strong contraction even though the oscillations are not slow-fast in the classical van der Pol-type sense.

\section{The Map $\Pi^{1}$ : Proof of Lemma 3.9}

We define additional transversal sections $\Sigma^{1, i}, i \in\{2,3,4,5\}$ as in Fig. 13, and consider the map $\Pi^{1}$ as a composition

$$
\Pi^{1}=\Pi^{1,5} \circ \Pi^{1,4} \circ \Pi^{1,3} \circ \Pi^{1,2} \circ \Pi^{1,1},
$$

where $\Pi^{1,1}: \Sigma^{1} \rightarrow \Sigma^{1,2}, \Pi^{1,5}: \Sigma^{1,5} \rightarrow \Sigma^{2}$, and $\Pi^{1, i}: \Sigma^{1, i} \rightarrow \Sigma^{1, i+1}$ for $i=2,3,4$ denote transition maps induced by the flow. To prove Lemma 3.9, we consider each map in turn. The first three mappings are more standard, so we will just summarise the findings.

\subsection{The Transition Maps $\Pi^{1, i}$ for $i=1,2,3$}

The mapping $\Pi^{1,1}:(x,-\delta, \epsilon) \mapsto\left(x^{1,1}(x, \epsilon),-\delta, \epsilon\right)$ is a diffeomorphism by regular perturbation theory. Notice that for any $c>0$, we have $\left|x^{1,1}(x, \epsilon)-x_{d}\right| \leq c$ for all $x \in[-\alpha, \alpha]$, $\epsilon \in\left[0, \epsilon_{0}\right]$ upon taking $\delta>0, \alpha>0$ and $\epsilon_{0}>0$ sufficiently small. Working in the chart $K_{1}$, see Lemma 3.3, the second (local) map $\Pi^{1,2}$ is also standard, see e.g. [18, Theorem 4.2], being of the following $C^{1}$ form $x \mapsto x^{1,2}(x)+\mathcal{O}\left(\epsilon \log \epsilon^{-1}\right)$. Here $x^{1,2}(x)$ is the base point of the local stable manifold intersecting $\Sigma^{1,2}$ at $(x,-\delta, 0)$, and the order $\mathcal{O}\left(\epsilon \log \epsilon^{-1}\right)$ does not change upon differentiation with respect to $x$. Working in chart $K_{2}, \Pi^{1,3}$ is a diffeomorphism of the form $x \mapsto x^{1,3}(x, \epsilon)$, with $x^{1,3}$ smooth and $x^{1,3}(x, 0)=x$ for all $x$. In total, the composition $\Pi^{1,3} \circ \Pi^{1,2} \circ \Pi^{1,1}$ is $C^{1}$ with respect to $x$ of the following form $x \mapsto \tilde{x}^{1,3}(x)+\mathcal{O}\left(\epsilon \log \epsilon^{-1}\right)$ with $\tilde{x}^{1,3}$ smooth.

The mappings $\Pi^{1,4}$ and $\Pi^{1,5}$ are less standard because they occur in the exponential regime. We therefore include some more details in the following.

\subsection{The Local Transition Map $\Pi^{1,4}: \Sigma^{1,4} \rightarrow \Sigma^{1,5}$}

We work in chart $\mathfrak{K}_{1}$, for which

$$
\Sigma^{1,4}=\left\{\left(x, r_{1}, \delta, e^{-\delta^{-1}}\right):\left|x_{d}-x\right| \leq \alpha_{4}, r_{1} \in\left[0, e^{\delta^{-1}} R_{4}\right]\right\},
$$

and define

$$
\Sigma^{1,5}=\left\{\left(x, R_{5}, \epsilon, \rho_{1}\right):\left|x_{d}-x\right| \leq \alpha_{5}, \epsilon \in\left[0, \beta_{5}\right], \rho_{1} \in\left[0, e^{-\beta_{5}^{-1}}\right]\right\},
$$




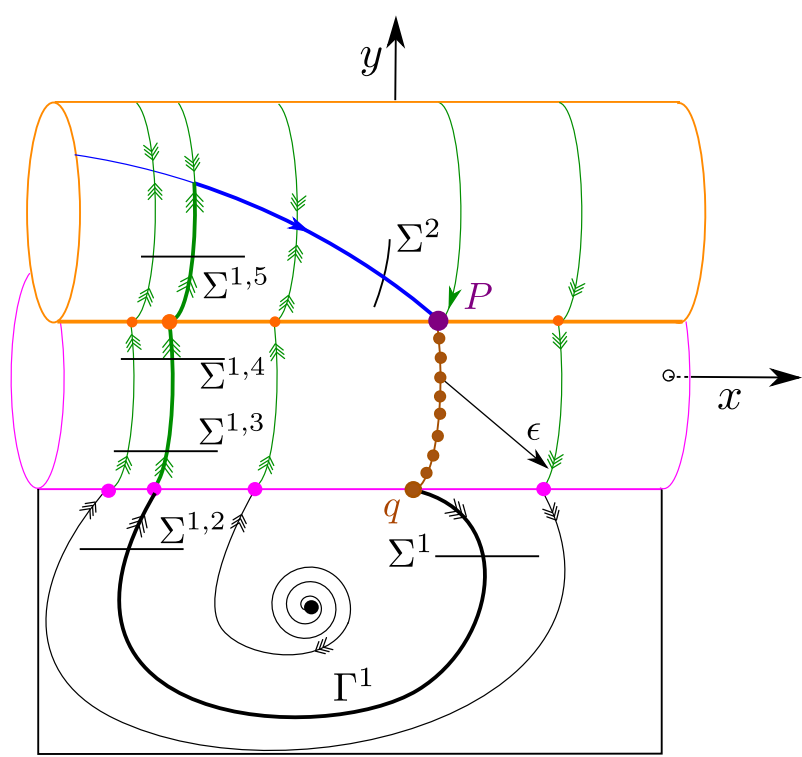

Fig. 13 To prove Lemma 3.9 we decompose $\Pi^{1}: \Sigma^{1} \rightarrow \Sigma^{2}$ into further transition mappings $\Pi^{1, i}, i=$ $1, \ldots, 5$. The relevant new sections $\Sigma^{1, i}, i=2, \ldots, 5$ are shown

as a small section about the intersection $\Gamma_{0} \cap\left\{r_{1}=R_{5}\right\}$. Some of the expressions simplify by setting

$$
R_{5}=e^{-\delta^{-1}}
$$

so we will adopt this choice in the following. The interval for $\rho_{1}$ is chosen by restriction to the invariant set $Q_{1}$. For ease of calculations, we drop the subscripts in (3.22) and translate $\left(x_{d}, 0,0,0\right)$ to the origin by introducing $\tilde{x}=x-x_{d}$. After dividing the right hand side of the new system by the locally positive factor $-\left(x_{d}+\tilde{x}+b r(1-2 b \rho)\right)$, we obtain the system

$$
\begin{aligned}
\tilde{x}^{\prime} & =\frac{-\rho r \epsilon(a+\rho r)}{x_{d}+\tilde{x}+b r(1-2 b \rho)}, \\
r^{\prime} & =r(1+\epsilon), \\
\epsilon^{\prime} & =-\epsilon^{2}, \\
\rho^{\prime} & =-\rho .
\end{aligned}
$$

We consider the system (4.2) in order to characterize the map $\Pi^{1,4}$.

Proposition 4.1 The map $\Pi^{1,4}: \Sigma^{1,4} \rightarrow \Sigma^{1,5}$ is well-defined for $\delta>0$ and $\alpha_{4}>0$ sufficiently small, and given by

$$
\Pi^{1,4}:\left(x, r, \delta, e^{-\delta^{-1}}\right) \mapsto\left(x+\mathcal{O}\left(r \log r^{-1}\right), e^{-\delta^{-1}}, W(1 / r \delta)^{-1}, r \delta W(1 / r \delta)\right) .
$$

Here the order of $\mathcal{O}\left(r \log r^{-1}\right)$ does not change by differentiation with respect to $x$. 
Proof Consider a solution of (4.2) satisfying

$$
\left\{\begin{array} { l } 
{ \tilde { x } ( 0 ) = \tilde { x } _ { \text { in } } , } \\
{ r ( 0 ) = r _ { \text { in } } , } \\
{ \epsilon ( 0 ) = \delta , } \\
{ \rho ( 0 ) = e ^ { - \delta ^ { - 1 } } , }
\end{array} \quad \left\{\begin{array}{l}
\tilde{x}(T)=\tilde{x}_{\text {out }}, \\
r(T)=R_{5}, \\
\epsilon(T)=\epsilon_{\text {out }}, \\
\rho(T)=\rho_{\text {out }} .
\end{array}\right.\right.
$$

The system

$$
\begin{aligned}
& r^{\prime}=r(1+\epsilon), \\
& \epsilon^{\prime}=-\epsilon^{2}, \\
& \rho^{\prime}=-\rho,
\end{aligned}
$$

decouples from (4.2), and can be integrated directly to give

$$
r(t)=r_{i n} e^{t}(1+\delta t), \quad \epsilon(t)=\frac{\delta}{1+\delta t}, \quad \rho(t)=e^{-\delta^{-1}} e^{-t} .
$$

One can use these equations to obtain the following expression for the transition time $T$ :

$$
T=-\delta^{-1}+W\left(\frac{R_{5}}{r_{i n} \delta e^{-\delta^{-1}}}\right) .
$$

By the above we obtain

$$
\epsilon(T)=W\left(\frac{R_{5}}{r_{i n} \delta e^{-\delta^{-1}}}\right)^{-1}, \quad \rho(T)=r_{i n} \delta e^{-\delta^{-1}} R_{5}^{-1} W\left(\frac{R_{5}}{r_{i n} \delta e^{-\delta^{-1}}}\right) .
$$

(These expressions also follow from the conservation of the original $\epsilon$ and the set $Q$, see (3.8) and (3.21): $r_{i n} \delta e^{-\delta^{-1}}=R_{5} \epsilon(T) e^{-\epsilon(T)^{-1}}$.) Furthermore,

$$
\left.\tilde{x}^{\prime}=\mathcal{O}(\rho(t) r(t) \epsilon(t))\right)=\mathcal{O}\left(r_{i n}\right),
$$

which is $C^{1}$-smooth, guaranteeing that the leading order is well behaved with respect to integration. Hence

$$
\tilde{x}_{\text {out }}-\tilde{x}_{\text {in }}=\mathcal{O}\left(r_{\text {in }} T\right) .
$$

The result then follows from (4.1) and using the asymptotics (1.18) of $W$.

\subsection{The Transition Map $\Pi^{1,5}: \Sigma^{1,5} \rightarrow \Sigma^{2}$}

We continue to work in chart $\mathfrak{K}_{1}$.

Proposition 4.2 For $\eta>0$ sufficiently small, the map $\Pi^{1,5}$ is well-defined and exponentially contracting in the $r_{1}$-coordinate. In particular, if $r_{1}^{1,5}\left(x, \epsilon, \rho_{1}\right)$ is the $r_{1}$-coordinate of $\Pi^{1,5}\left(x, R_{5}, \epsilon, \rho_{1}\right)$ then $r_{1}^{1,5}$ is $C^{1}$ with respect to $x$, satisfying the following estimates

$$
\begin{aligned}
r_{1}^{1,5}\left(x, \epsilon, \rho_{1}\right) & =\frac{\eta}{b}+\mathcal{O}\left(\rho_{1}\right), \\
\frac{\partial}{\partial x} r_{1}^{1,5}\left(x, \epsilon, \rho_{1}\right) & =\mathcal{O}\left(e^{-c /\left(\epsilon \rho_{1}\right)}\right),
\end{aligned}
$$

for some $c>0$. 
Proof Trajectories with initial conditions in $\Sigma^{1,5}$ are strongly attracted to their base-points on the invariant center manifold $M_{1}$ in Lemma 3.7, and track the slow flow after reaching a local tubular neighborhood of $M_{1}$. To leading order, the flow on $M_{1}$ is determined by

$$
\left.x^{\prime}=-\frac{a}{b} x \epsilon \rho_{1}\left(1+\mathcal{O}\left(\epsilon+\rho_{1}\right)\right)\right),
$$

so that $x^{\prime}>0$ and $\Pi^{1,5}$ is therefore well-defined. Center manifold theory implies exponential contraction $\left(e^{-c t}\right)$ along one-dimensional fibers with base points on $M_{1}$. Since

- $r_{1}(t) \geq v>0$ for some $v>0$ sufficiently small during the transition from $\Sigma^{1,4}$ to $\Sigma^{1,5}$; - $r_{1} \rho_{1} \epsilon=$ const., see (3.8) and (3.21);

we can estimate the travel time, where $x$ changes by $\mathcal{O}(1)$, to be of order $\mathcal{O}\left(1 /\left(\epsilon \rho_{1}\right)\right)$. The result therefore follows.

\subsection{Proof of Lemma 3.9}

The analysis of the mappings $\Pi^{1, i}, i \in\{1,2,3,4,5\}$ in Sects. $4.1,4.2$ and 4.3 prove Lemma 3.9 upon composition. In particular, the exponential contraction in the $r_{1}$-coordinate in Lemma 3.9, is a corollary of Proposition 4.2 upon using that $\epsilon=r_{1} \rho_{1} \epsilon_{3}$, see (3.8) and (3.21), with $r_{1} \approx \eta / b$.

\section{The Map $\Pi^{2}$ : Proof of Lemma 3.10}

The analysis of the map $\Pi^{2}$ requires good control of the flow close to the line $L$ of degenerate equilibria $L$, see Fig. 12. Additional blow-ups are necessary in order to prove Lemma 3.10. In this section we summarise the blow-up transformations and dynamical features leading to the construction of the final nondegenerate singular cycle $\Gamma_{0}$ as shown in Fig. 14. The cycle $\Gamma_{0}$ has a total of eight distinct segments $\Gamma^{i}, i \in\{1, \ldots, 8\}$. We have already identified the segments $\Gamma^{i}$ for $i \in\{1,2,3,4\}$ in (3.35); the segments $\Gamma^{i}$ for $i \in\{5,6,7,8\}$ will be defined by three additional blow-ups needed to resolve the degeneracies of the line $L$. This section is included here for expository purposes: a more detailed technical presentation is given in Sect. 6.

By looking at Fig. 12 and by extrapolating from what we did so far, it is natural to expect that a straightforward (cylindrical) blow-up of the non-hyperbolic line (circle) $L$ will be necessary for the construction of the final singular cycle $\Gamma_{0}$. However, it turns out that additional difficulties arise close to the point $P$ which is the endpoint of $L$ on the (orange) cylinder, corresponding to $\rho=0$. This is somewhat expected, given that this transition corresponds dynamically to a transition between regimes in which exponential terms are dominant (the upper horizontal cylinder) to a regime in which algebraic terms are dominant (the vertical cylinder obtained after blow-up of $L$ ). The analysis of this transition requires two additional spherical blow-ups shown in Fig. 14, which are necessary for resolving the degeneracies and 'connecting' the two regimes.

\subsection{Successive Blow-Ups and the Fully Nondegenerate Singular Cycle $\Gamma_{0}$}

The required sequence of blow-up transformations is sketched in Fig. 15, and outlined in the following. 


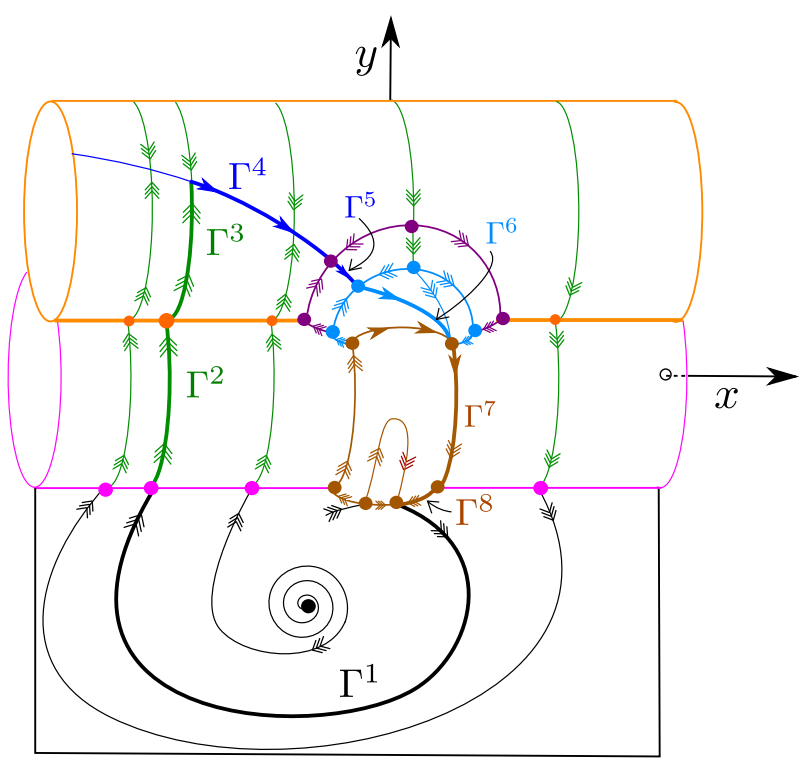

Fig. 14 The fully nondegenerate singular cycle obtained from the repeated blow-up transformations, visualized in Fig. 15, summarized in Sect. 5.1 and detailed in Sect. 6

By Lemma 3.5, Sect. 3.1, the manifold $\mathcal{C}$ terminates in a non-hyperbolic point $P$ (purple) at the endpoint of the non-hyperbolic curve $L_{e}$ (the origin in chart $\mathfrak{K}_{1}$ coordinates) corresponding to the endpoint of the vertical non-hyperbolic line $L$ (brown); see Fig. 15a. In order to resolve this degeneracy, this point is blown-up to a sphere as in Fig. 15b; this is done in Sect. 6.1.1 as part of a larger 'cylinder of spheres' blow-up. Note that this sphere is the 'outer sphere' (also purple) in Fig. 14. The manifold $\mathcal{C}$ then terminates in a partially hyperbolic and attracting point $P_{L}$ on the equator of this sphere. One identifies the extension of the singular cycle as a normally hyperbolic attracting curve of equlibria $\mathcal{N}$ (blue) on this sphere, terminating in another non-hyperbolic point $P_{O}$ (cyan), as shown in Fig. 15b.

The non-hyperbolic point $P_{O}$ is analyzed in Sect. 6.1 .3 by means of a second spherical blow-up. This is the 'inner sphere' (also cyan) sketched in Figs. 14 and 15c. We identify an attracting, partially hyperbolic point $p_{l}$ (cyan) on the equator of the sphere as the endpoint of $\mathcal{N}$. The relevant thing for the construction of $\Gamma_{0}$ is the existence of a unique center manifold $\mathcal{W}$ (cyan) emanating from $p_{l}$, which we can identify with $\Gamma^{6}$; compare Figs. 14 and 15 c. Phase plane arguments (the system is no longer slow-fast) are given in Sect. 6.1.3 (see Lemma 6.6 in particular) in order to show that $\mathcal{W}$ terminates in yet another non-hyperbolic point $p_{O}$ (brown), from which the non-hyperbolic line $L$ (also brown) emanates.

All remaining non-hyperbolicity is resolved by means of cylindrical blow-up of $L$ in Sects. 6.1.4 and 6.2, leading to the fully resolved scenario sketched in Fig. 14, see also Fig. 15d. In Sect. 6.1.4, a cylindrical blow-up applied locally near the inner blow-up sphere leads to the identification of a partially hyperbolic saddle point $p_{s}$ (brown). The orbit $\mathcal{W}$ terminates at $p_{s}$, and the next component of $\Gamma_{0}$ can be identified with the unstable manifold $W^{u}\left(p_{s}\right)$, which is shown in Lemma 6.7 to lie along the outside 'edge' of the cylinder, denoted by $\mathcal{H}$ (also brown) in Fig. 15d. The remaining analysis is carried out in Sect. 6.2, after it is shown in Lemma 6.8 that the dynamics in the local cylindrical blow-up described above can be mapped to those obtained in a cylindrical blow-up of the line $L$ in the algebraic regime, 


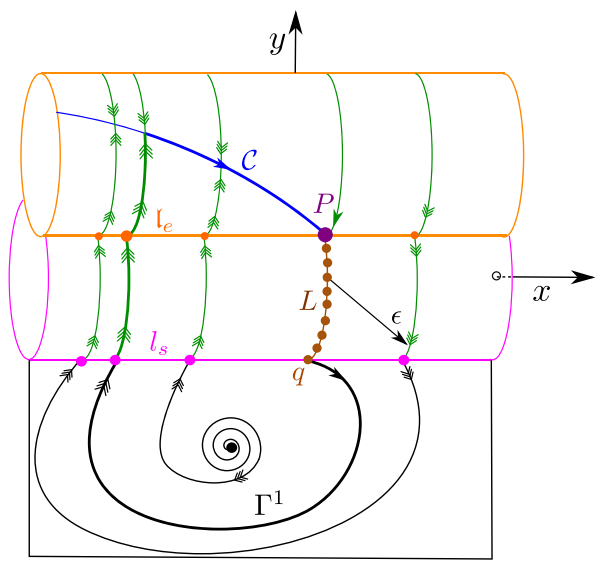

(a)

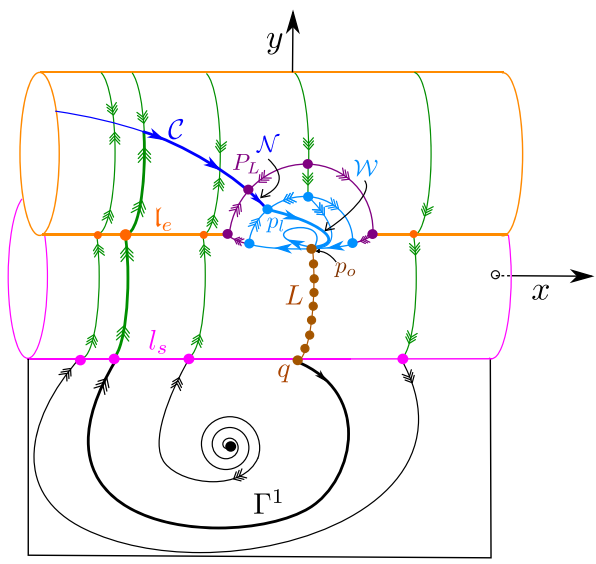

(c)

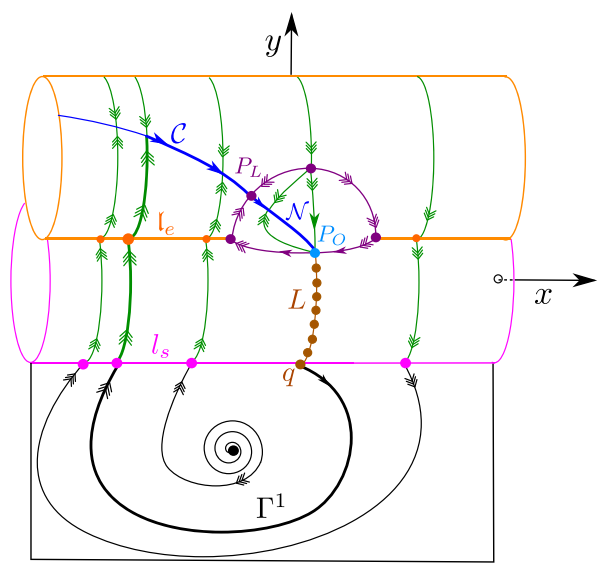

(b)

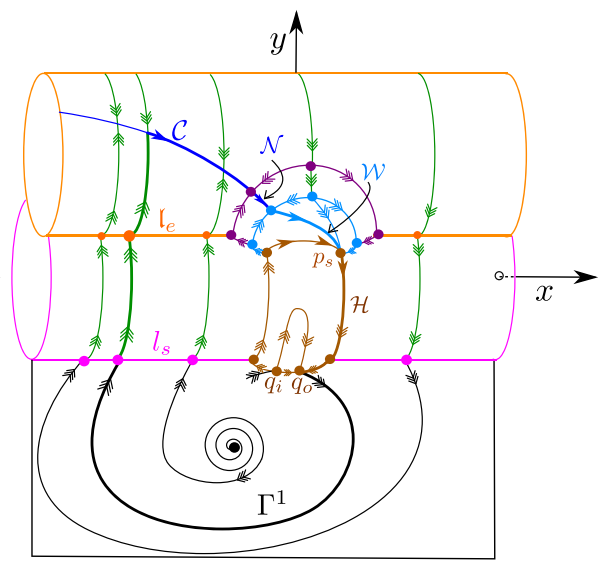

(d)

Fig. 15 Sequence of blow-up transformations needed to desingularize the dynamics near the line of degenerate equilibria $L$ and to connect the exponential regime and the critical manifold $\mathcal{C}$ with the orbit $\Gamma^{1}$ (black) in the plane $\bar{\epsilon}=0$. In a we show again Fig. 11. The degenerate equilibrium $P$ (purple) is blown up to a sphere (purple) in $\mathbf{b}$, as described in Sect. 6.1.1. On this sphere another degenerate equilibrium $P_{O}$ (cyan) is identified, which is blown-up to another sphere (cyan) shown in c, as detailed in Sect. 6.1.3. Finally, the still remaining line of degenerate equilibria $L$ (brown) is blown-up to a cylinder (brown) shown in $\mathbf{d}$ and analyzed in Sects. 6.1.4 and 6.2 (Color figure online)

i.e. in charts $K_{i}, i=1,2,3$, subsequent to the (first) cylindrical blow-up leading to the lower horizontal cylinder in Fig. 14. The analysis leads to the identification of the final two cycle segments $\Gamma^{7}$ and $\Gamma^{8}$ in Fig. 14. The first can be identified with $\mathcal{H}$, which is shown in Lemma 6.13 to be invariant and terminating in a partially hyperbolic singularity at $q_{s}$. The second can be identified with the unstable manifold $W^{u}\left(q_{s}\right)$, which is invariant, and shown (also in Lemma 6.13) to terminate in a hyperbolic (resonant) saddle singularity $q_{o}$. Although the analysis here is to some extent standard, it is frequently complicated by the fact that transition across different coordinate charts becomes non-trivial after the application of multiple blow-up transformations. 


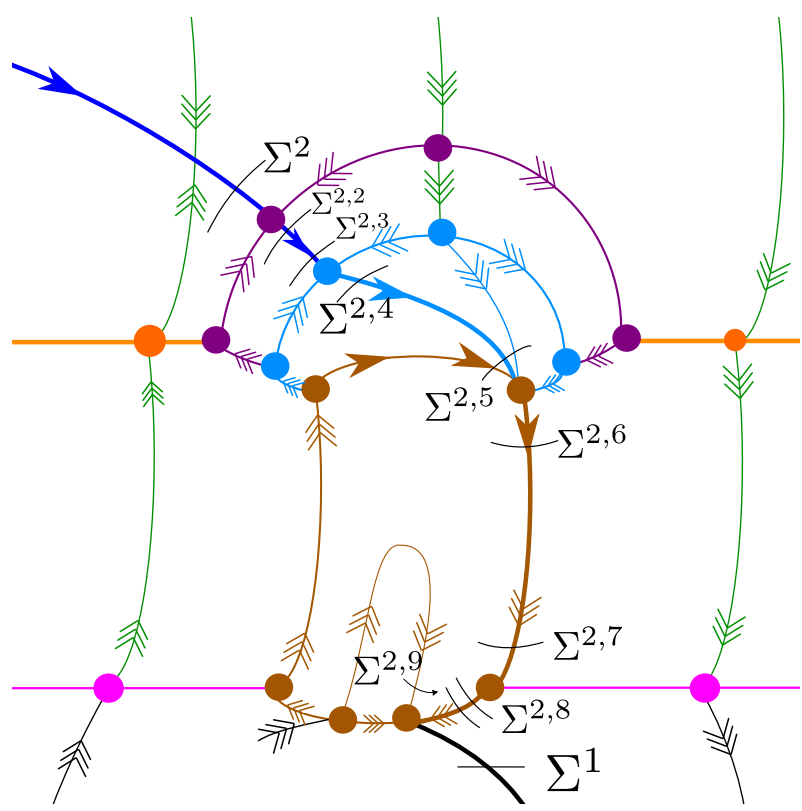

Fig. 16 Illustration of the sections $\Sigma^{2, i}, i \in\{2, \ldots, 9\}$ relevant for the description of the map $\Pi^{2}$ as the composition of several transition maps $\Pi^{2, i}, i \in\{1, \ldots, 9\}$

To summarise, we define the fully nondegenerate singular cycle $\Gamma_{0}$ by

$$
\Gamma_{0}=\Gamma^{1} \cup \Gamma^{2} \cup \Gamma^{3} \cup \Gamma^{4} \cup \Gamma^{5} \cup \Gamma^{6} \cup \Gamma^{7} \cup \Gamma^{8},
$$

where $\Gamma^{i}$ for $i \in\{1,2,3,4\}$ have already been defined in (3.35) and where

$$
\left\{\begin{array}{l}
\Gamma^{5}=\mathcal{N}, \\
\Gamma^{6}=\mathcal{W}, \\
\Gamma^{7}=\mathcal{H}, \\
\Gamma^{8}=\left\{\left(r_{11}, 0,0\right): r_{11} \in\left[0, \frac{1}{2 a}\right]\right\}, \quad \text { chart } K_{11} .
\end{array}\right.
$$

Regarding $\Gamma^{8}$, the coordinates specified by the chart $K_{11}$ are defined in Sect. 6.2.6, see also (6.40). Note also that the union $\Gamma^{5} \cup \Gamma^{6} \cup \Gamma^{7} \cup \Gamma^{8}$ of the segments in (5.1) becomes $L$ in (3.35) upon blowing down. Each segment has improved hyperbolicity properties that allow us to describe the mapping $\Pi^{2}$ and prove Lemma 3.10.

\subsection{Proof of Lemma 3.10: A Summary}

Similar to our analysis of the map $\Pi^{1}$, the map $\Pi^{2}$ can be analyzed by defining additional transversal sections $\Sigma^{2, i}, i \in\{2, \ldots, 9\}$ as in Fig. 16, and considering the composition

$$
\Pi^{2}=\Pi^{2,9} \circ \Pi^{2,8} \circ \Pi^{2,7} \circ \Pi^{2,6} \circ \Pi^{2,5} \circ \Pi^{2,4} \circ \Pi^{2,3} \circ \Pi^{2,2} \circ \Pi^{2,1} .
$$

Here $\Pi^{2,1}: \Sigma^{2} \rightarrow \Sigma^{2,2}, \Pi^{2,9}: \Sigma^{2,9} \rightarrow \Sigma^{1}$, and $\Pi^{2, i}: \Sigma^{2, i} \rightarrow \Sigma^{2, i+1}$ for $i \in\{2, \ldots 8\}$ denote transition maps induced by the flow.

To prove Lemma 3.10, we have to consider the maps $\Pi^{2, i}$ in turn. However, each mapping is standard so we just focus on explaining in what coordinates the mappings are described, 
making references to equations, sections and lemmas to come below, and summarise the findings as follows:

The transition map $\Pi^{2,1}$ is described in the coordinates specified by the chart $\mathcal{K}_{1}$, see (6.3). By center manifold theory and Lemma 6.2 below the mapping is well-defined and exponentially contracting in the direction transverse to $\mathcal{W}$.

The transition map $\Pi^{2,2}$ is described in the coordinates specified by the chart $\mathcal{K}_{2}$, see (6.3). The properties of this map are similar to $\Pi^{2,1}$ since the line of partially hyperbolic equilibria $\mathcal{N}$ extends all the way down to $P_{O}$. However, $P_{O}$ is non-hyperbolic and it is important to highlight that in $\mathcal{K}_{2}$ we are leaving the 'exponential regime'. We can therefore reduce the phase space dimension again, see (6.7). This produces a 3-dimensional system.

The transition map $\Pi^{2,3}$ is described in the coordinates specified by the chart $\widetilde{\mathcal{K}}_{1}$, see (6.16). Center manifold theory and Lemma 6.5 below show that the mapping is well-defined and exponentially contracting in the direction transverse to $\mathcal{W}$.

The transition map $\Pi^{2,4}$ is a diffeomorphism by regular perturbation theory applied in the coordinates specified by the chart $\widetilde{\mathcal{K}}_{2}$, see (6.16).

The transition map $\Pi^{2,5}$ is described in the coordinates specified by the chart $\widehat{K}_{31}$, see (6.23) and the equations of motion in (6.24), associated with the blow-up of $L$. In these coordinates we gain hyperbolicity, as indicated in Fig. 15d, see also the local picture in Fig. 21. Within the center manifold, the point $p_{s}$ (see Fig. 15d) is a hyperbolic saddle upon further desingularization, having $\mathcal{H}$ as an unstable manifold. The mapping $\Pi^{2,5}$ is therefore well-defined and (algebraically) contracting.

The transition map $\Pi^{2,6}$. By Lemma 6.8 below, we can transform the result on $\Pi^{2,5}$ into the chart $K_{21}$, see (6.28), associated with blowing up $L$ in the original scaling chart $K_{2}$, recall (3.7). Here we can also describe the mapping $\Pi^{2,6}$ as a diffeomorphism by regular perturbation theory using the invariance of the line $\mathcal{H}$. See also Lemma 6.11 .

The transition map $\Pi^{2,7}$ is described in the coordinates specified by the chart $K_{11}$, see (6.27), as a local passage near the semi-hyperbolic saddle $q_{s}$. The mapping is well-defined and non-expanding; the details being similar to those in [18, Theorem 4.2].

The transition map $\Pi^{2,8}$ is described in the coordinates specified by the chart $K_{11}$, see (6.27). Since the flow along the invariant line between $\Gamma^{8} \cap \Sigma^{2,8}$ and $\Gamma^{8} \cap \Sigma^{2,9}$ is regular and finite-time (see Figs. 14 and 16), $\Pi^{2,8}$ is a diffeomorphism.

The transition map $\Pi^{2,9}$ is described in the coordinates specified by the chart $K_{12}$, see (6.27), as passage near the resonant hyperbolic saddle $q_{o}$ with a one dimensional unstable manifold $\Gamma^{1}$. The details are similar to those in [22, lemma 3.6].

Each map is one-dimensional and at least $C^{1}$ upon restriction to $0<\epsilon \ll 1$ and the set $Q$. This completes the (sketched) proof of Lemma 3.10 upon composition.

\section{Blow-Up Analysis for the Map $\Pi^{2}$}

The blow-up transformations and main features in the analysis for the proof of Lemma 3.10 are presented in this section. Our analysis divides into two parts: (i) an understanding of the transition between the exponential and algebraic regimes, and (ii) a blow-up analysis describing the dynamics near the non-hyperbolic line $L$ in the algebraic regime. Part (i) is considered in Sect. 6.1, and focuses (among other things) on the spherical blow-ups shown in Fig. 14. Part (ii) is considered in Sect. 6.2. 


\subsection{Exiting the Exponential Regime}

Here we consider the transition out of the 'exponential regime'. In terms of Fig. 14, our aim is to understand the manner in which trajectories move from the upper horizontal cylinder (exponential regime), to the vertical cylinder (algebraic regime).

\subsubsection{Blow-Up Near the Non-hyperbolic line $L_{e, 1}$ in Chart $\mathfrak{K}_{1}$}

We start in the exponential regime, chart $\mathfrak{K}_{1}$, dropping the subscripts in system (3.22):

$$
\begin{aligned}
x^{\prime} & =\rho r \epsilon(a+\rho r), \\
r^{\prime} & =-r(1+\epsilon)(x+b r(1-2 \rho)), \\
\epsilon^{\prime} & =\epsilon^{2}(x+b r(1-2 \rho)), \\
\rho^{\prime} & =\rho(x+b r(1-2 \rho)) .
\end{aligned}
$$

By Lemma 3.5, the one-dimensional manifold $\mathcal{C}$ identified with the cycle segment $\Gamma^{4}$ terminates at $(0,0,0,0) \in \mathcal{P}$, where $\mathcal{P}: x=r=0$ constitutes an entire plane of non-hyperbolic fixed points for (6.1). One could proceed by blowing up the entire plane $\mathcal{P}$, however only the dynamics near the curve $L_{e} \subset \mathcal{P}$ will be relevant for the transition (recall that $L_{e}$ and $L_{3}$ coincide where domains overlap; see again Lemma 3.5). This motivates a blow-up of $(x, r, \rho)=(0,0,0)$ in the following form

$$
v \geq 0,(\bar{x}, \bar{r}, \bar{\rho}) \in S^{2} \mapsto\left\{\begin{array}{l}
x=v \bar{x}, \\
r=v \bar{r}, \\
\rho=v \bar{\rho} .
\end{array}\right.
$$

We introduce the coordinate charts

$$
\mathcal{K}_{1}: \bar{x}=-1, \quad \mathcal{K}_{2}: \bar{\rho}=1,
$$

for which we have chart-specific coordinates given by

$$
\begin{aligned}
& \mathcal{K}_{1}: x=-v_{1}, \quad r=v_{1} r_{1}, \quad \rho=v_{1} \rho_{1}, \\
& \mathcal{K}_{2}: x=\nu_{2} x_{2}, \quad r=v_{2} r_{2}, \quad \rho=\nu_{2} .
\end{aligned}
$$

Remark 6.1 Notice that $\epsilon$ is not transformed by (6.2). Geometrically, (6.2) therefore blows up

$$
\mathcal{L}=\{(0,0, \epsilon, 0): \epsilon \geq 0\} \subset \mathcal{P},
$$

to a 'cylinder of spheres' $C S=\{v=0\} \times S^{2} \times \mathbb{R}$. Note that each $C S \cap\{\epsilon=$ const. $\}$ is an invariant sphere in $C S$. Notice also that

(I) $L_{e}$ and $\mathcal{L}$ are tangent at $(0,0,0,0)$, and

$$
L_{e} \cap \mathcal{L}=\{(0,0,0,0)\} .
$$

(II) Considered in terms of its parameterization (3.23), $L_{e}$ is flat at $(0,0,0,0)$, and thus flat with respect to the line $\mathcal{L}$ as $\epsilon \rightarrow 0$; see Fig. 17 .

Geometrically, it seems more natural to blow-up $L_{e}$. To do this one would rectify $L_{e}$ and apply the cylindrical blow-up transformation (6.2) along (the transformed) $L_{e}$. However, our approach avoids this unnecessary coordinate transformation. Besides (a) only the sphere 
Fig. 17 Illustration within $\{x=0\}$ of the lines $\mathcal{L}$ (magenta) and $L_{e}$ (brown) as subsets of the plane $\mathcal{P}: x=r=0$ (shaded magenta) (Color figure online)

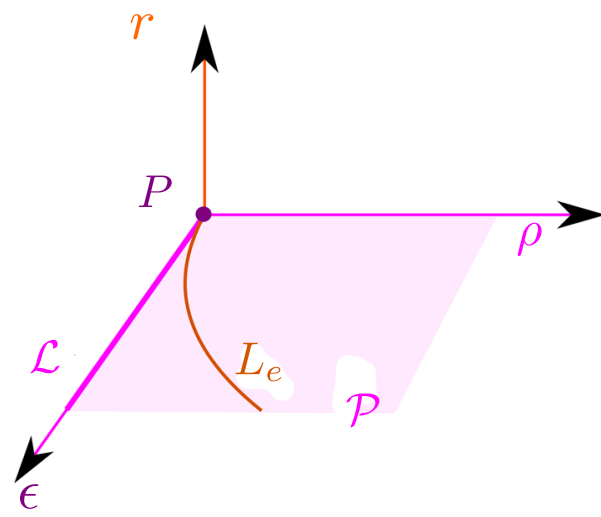

$C S \cap\{\epsilon=0\}$, which is the same for both approaches (recall (6.5)), will be relevant, and (b) once we leave the exponential regime and enter $\bar{\rho}>0$ of the sphere (6.2), we will apply a subsequent blow-up transformation that effectively blows up $L_{e}$ (or $L$ ).

We will adopt the following notational convention: given an object $\gamma$ identified in chart $\mathcal{K}_{1}$, we denote its image under the blow-up transformation (6.2) by $\gamma^{\prime}$, and it's image in a particular coordinate chart $\mathcal{K}_{i}$ by $\gamma_{i}^{\prime}$ (this will help to avoid confusion given the earlier dropping of subscripts etc).

The transition maps between coordinates in charts $\mathcal{K}_{i}, i=1,2$ are given by

$$
\begin{array}{llll}
\kappa_{12}^{\prime}: r_{1}=-x_{2}^{-1} r_{2}, & \rho_{1}=-x_{2}^{-1}, & v_{1}=-v_{2} x_{2}, & x_{2}<0, \\
\kappa_{21}^{\prime}: x_{2}=-\rho_{1}^{-1}, & r_{2}=\rho_{1}^{-1} r_{1}, & v_{2}=v_{1} \rho_{1}, & \rho_{1}>0 .
\end{array}
$$

Recall also that the set $Q=\left\{(x, r, \epsilon, \rho): \rho=e^{-\epsilon^{-1}}\right\}$ is invariant for the system (6.1). In chart $\mathcal{K}_{i}$ coordinates, then, the following are invariant:

$$
Q_{1}^{\prime}=\left\{\left(r_{1}, \epsilon, \rho_{1}, v_{1}\right): v_{1} \rho_{1}=e^{-\epsilon^{-1}}\right\}, \quad Q_{2}^{\prime}=\left\{\left(x_{2}, r_{2}, \epsilon, v_{2}\right): v_{2}=e^{-\epsilon^{-1}}\right\} .
$$

\section{$\mathcal{K}_{1}$ Chart}

The dynamics in chart $\mathcal{K}_{1}$ are governed by

$$
\begin{aligned}
& r_{1}^{\prime}=r_{1}\left((1+\epsilon)\left(1-b r_{1}\left(1-2 v_{1} \rho_{1}\right)\right)+\rho_{1} r_{1} \epsilon\left(a+\rho_{1} r_{1} v_{1}^{2}\right)\right), \\
& \epsilon^{\prime}=-\epsilon^{2}\left(1-b r_{1}\left(1-2 v_{1} \rho_{1}\right)\right), \\
& \rho_{1}^{\prime}=-\rho_{1}\left(1-b r_{1}\left(1-2 v_{1} \rho_{1}\right)-\rho_{1} r_{1} \epsilon\left(a+\rho_{1} r_{1} v_{1}^{2}\right)\right), \\
& v_{1}^{\prime}=-v_{1} \rho_{1} r_{1} \epsilon\left(a+\rho_{1} r_{1} v_{1}^{2}\right) .
\end{aligned}
$$

after a suitable desingularization (division by $v_{1}$ ). The manifold of equilibria $C_{1}$ in Lemma 3.5 shows up as a manifold of equilibria $C_{1}^{\prime}$ for the system in the invariant hyperplane $\epsilon=0$ given by

$$
\begin{aligned}
& r_{1}^{\prime}=r_{1}\left(1-b r_{1}\left(1-2 v_{1} \rho_{1}\right)\right), \\
& \rho_{1}^{\prime}=-\rho_{1}\left(1-b r_{1}\left(1-2 v_{1} \rho_{1}\right)\right), \\
& v_{1}^{\prime}=0,
\end{aligned}
$$


and the manifold of equilbira $S_{1}$ in Lemma 3.5 shows up as a manifold of equilibria $S_{1}^{\prime}$ for the system in the invariant hyperplane $\rho_{1}=0$, given by

$$
\begin{aligned}
r_{1}^{\prime} & =r_{1}\left((1+\epsilon)\left(1-b r_{1}\right)\right), \\
\epsilon^{\prime} & =-\epsilon^{2}\left(1-b r_{1}\right), \\
v_{1}^{\prime} & =0 .
\end{aligned}
$$

We are interested in the intersection $C_{1}^{\prime} \cap S_{1}^{\prime}$, since this is contained within the domain of interest $Q_{1}^{\prime}$. In particular, this intersection constitutes a line of equilbria

$$
\mathcal{C}_{1}^{\prime}=C_{1}^{\prime} \cap Q_{1}^{\prime}=S_{1}^{\prime} \cap Q_{1}^{\prime}=C_{1}^{\prime} \cap S_{1}^{\prime}=\left\{\left(b^{-1}, 0,0, v_{1}\right): v_{1} \geq 0\right\},
$$

in the $\epsilon=\rho_{1}=0$ plane. The line $\mathcal{C}_{1}^{\prime}$ terminates at the point $P_{L}:\left(b^{-1}, 0,0,0\right)$, which sits on the equator of the invariant sphere segment $v_{1}=\epsilon=0$. In fact, within the invariant plane $v_{1}=\epsilon=0$ we have

$$
\begin{aligned}
& r_{1}^{\prime}=r_{1}\left(1-b r_{1}\right), \\
& \rho_{1}^{\prime}=-\rho_{1}\left(1-b r_{1}\right),
\end{aligned}
$$

which has a line of equilibria

$$
\mathcal{N}_{1}^{\prime}=\left\{\left(b^{-1}, 0, \rho_{1}, 0\right): \rho_{1} \geq 0\right\}
$$

emanating from $P_{L}$. Finally, we identify a line of equilibria along the positive $v_{1}$-axis, i.e. along

$$
\mathfrak{l}_{e, 1}^{\prime}=\left\{\left(0,0,0, v_{1}\right): v_{1} \geq 0\right\}
$$

Lemma 6.2 The following hold for system (6.8):

(i) The point $P_{L}$ is partially hyperbolic with a single non-zero eigenvalue $\lambda=-1$, and there exists a corresponding three-dimensional center manifold $M_{1}^{\prime}$ tangent to the center eigenspace $E^{c}\left(P_{L}\right)$, which can be chosen to be the extension of the manifold $M_{1}$.

Locally, $M_{1}^{\prime}$ contains the two-dimensional manifolds of equilbria $C_{1}^{\prime}$ and $S_{1}^{\prime}$ as restrictions

$$
\left.M_{1}^{\prime}\right|_{\epsilon=0}=C_{1}^{\prime},\left.\quad M_{1}^{\prime}\right|_{\rho_{1}=0}=S_{1}^{\prime},
$$

and the one-dimensional manifolds of equilbria $\mathcal{C}_{1}^{\prime}$ and $\mathcal{N}_{1}^{\prime}$ as restrictions

$$
\left.M_{1}^{\prime}\right|_{\epsilon=\rho_{1}=0}=\mathcal{C}_{1}^{\prime},\left.\quad M_{1}^{\prime}\right|_{\epsilon=\nu_{1}=0}=\mathcal{N}_{1}^{\prime} .
$$

The variables $\epsilon, \rho_{1}\left(r_{1}\right)$ are increasing (decreasing) along $M_{1}^{\prime} \backslash\left(C_{1}^{\prime} \cup S_{1}^{\prime} \cup \mathcal{N}_{1}^{\prime}\right)$.

(ii) The eigenvalues associated with the linearization along $\mathfrak{l}_{e, 1}^{\prime}$ are given by

$$
\lambda=1,0,-1,0,
$$

implying that $\mathfrak{l}_{e, 1}^{\prime}$ is a line of partially hyperbolic saddles.

Proof Assertion (i) is standard center manifold theory. Assertion (ii) is a direct calculation. 


\subsection{2 $\mathcal{K}_{2}$ Chart}

The system in chart $\mathcal{K}_{2}$ is given by

$$
\begin{aligned}
x_{2}^{\prime} & =-x_{2}\left(x_{2}+b r_{2}\left(1-2 v_{2}\right)\right)+r_{2} \epsilon\left(a+r_{2} v_{2}^{2}\right), \\
r_{2}^{\prime} & =-r_{2}(2+\epsilon)\left(x_{2}+b r_{2}\left(1-2 v_{2}\right)\right), \\
\epsilon^{\prime} & =\epsilon^{2}\left(x_{2}+b r_{2}\left(1-2 v_{2}\right)\right), \\
v_{2}^{\prime} & =v_{2}\left(x_{2}+b r_{2}\left(1-2 v_{2}\right)\right),
\end{aligned}
$$

after a suitable desingularization (division by $v_{2}$ ). At this point we note that the analysis may be simplified by restricting to the invariant set $Q_{2}^{\prime}$. By doing so we reduce the dimension by 1 after eliminating $v_{2}$, and consider the reduced system on this set

$$
\begin{aligned}
& x_{2}^{\prime}=-x_{2}\left(x_{2}+b r_{2}\left(1-2 e^{-\epsilon^{-1}}\right)\right)+r_{2} \epsilon\left(a+r_{2} e^{-2 \epsilon^{-1}}\right), \\
& r_{2}^{\prime}=-r_{2}(2+\epsilon)\left(x_{2}+b r_{2}\left(1-2 e^{-\epsilon^{-1}}\right)\right), \\
& \epsilon^{\prime}=\epsilon^{2}\left(x_{2}+b r_{2}\left(1-2 e^{-\epsilon^{-1}}\right)\right) .
\end{aligned}
$$

Note that the system obtained by restricting to $\epsilon=0$ in (6.13) is equivalent to the system obtained by restricting to $\nu_{2}=\epsilon=0$ in system (6.12), and given by

$$
\begin{aligned}
& x_{2}^{\prime}=-x_{2}\left(x_{2}+b r_{2}\right), \\
& r_{2}^{\prime}=-2 r_{2}\left(x_{2}+b r_{2}\right) .
\end{aligned}
$$

Here we identify the line of equilbria

$$
\mathcal{N}_{2}^{\prime}=\left\{\left(x_{2},-b^{-1} x_{2}, 0\right): x_{2} \leq 0\right\}
$$

corresponding to the extension of $\mathcal{N}^{\prime}$ in chart $\mathcal{K}_{2}$, as well as a line of equilibria

$$
L_{e, 2}^{\prime}=\{(0,0, \epsilon): \epsilon \geq 0\} .
$$

Lemma 6.3 The following holds for the system (6.13):

(i) The line of equlibria $\mathcal{N}_{2}^{\prime}$ has a single non-trivial eigenvalue $\lambda=x_{2} \leq 0$. Hence $\mathcal{N}_{2}^{\prime}$ is normally hyperbolic and attracting for $x_{2}<0$, and terminates in a non-hyperbolic point at the origin $P_{O}:(0,0,0)$.

(ii) There exists a unique center manifold $M_{2}^{\prime}$ with base along compact subsets of $\mathcal{N}_{2}$ bounded away from $P_{O}$. The manifold $M_{2}^{\prime}$ can be identified with the extension of the manifold $M_{1}^{\prime}$ identified in chart $\mathcal{K}_{1}$ coordinates in Lemma 6.2. The variable $r_{2}$ is decreasing along $M_{2}^{\prime} \backslash \mathcal{N}_{2}^{\prime}$.

(iii) The line $L_{e, 2}^{\prime}$ is non-hyperbolic, and coincides where domains overlap with the nonhyperbolic line $L_{3}$ identified in chart $K_{3}$ coordinates.

Proof Statement (i) follows immediately after linearization of the system (6.14) along $\mathcal{N}_{2}^{\prime}$.

The statement (ii) follows from center manifold theory, together with uniqueness of the manifold $M_{1}^{\prime}$ described in Lemma 6.2 and an application of the transition map $\kappa_{12}^{\prime}$ in (6.6).

The statement (iii) follows after linearization of the system (6.13) and an application of the blow-down transformation.

The situation is illustrated in Fig. 18. 


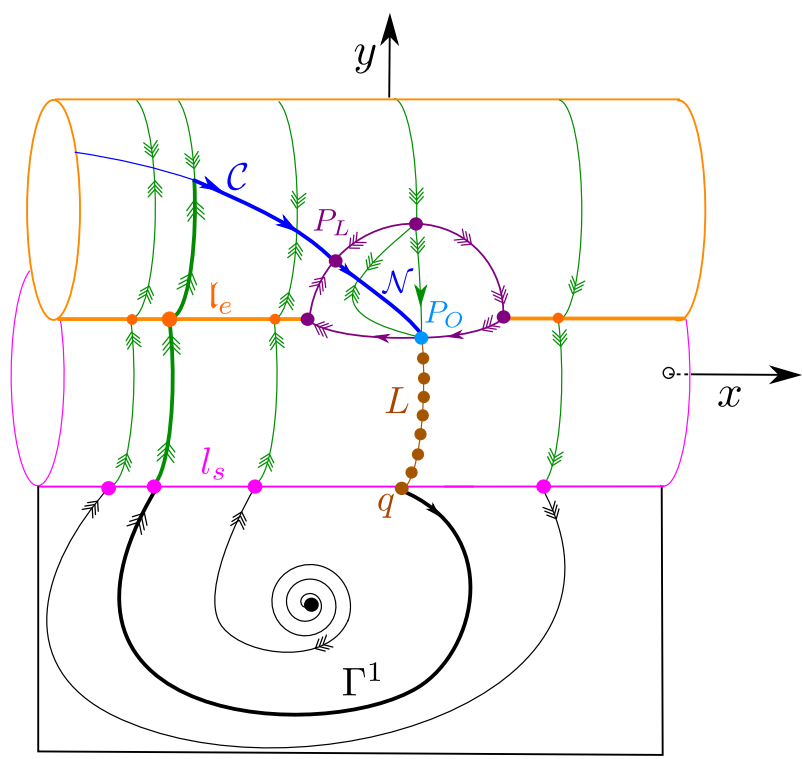

Fig. 18 Illustration of the blowup of the degenerate equilibrium $P$ (purple) to the 'outer sphere' (purple) within $\bar{\epsilon}=0$. On the sphere we find the attracting critical manifold $\mathcal{N}$ (blue) with reduced flow connecting $P_{L}$ to the degenerate equilibrium $P_{O}$ (cyan). The fast flow on the sphere is shown in green (Color figure online)

Remark 6.4 Figure 18 shows dynamics not visible in charts $\mathcal{K}_{1}$ or $\mathcal{K}_{2}$. To gain a complete picture of the dynamics one must look in the additional coordinate charts $\mathcal{K}_{3}: \bar{r}=1$ and $\mathcal{K}_{4}: \bar{x}=1$. In particular, a hyperbolic saddle $P_{R}$ is identified at $(\bar{x}, \bar{r}, \bar{\rho})=(0,1,0), \epsilon=0$ in chart $\mathcal{K}_{3}$, and a partially hyperbolic line of saddle-type equlibria is identified along the positive $\bar{x}$-axis in chart $\mathcal{K}_{4}$. We omit the details here for expository reasons.

\subsubsection{Spherical Blow-Up of the Point $P_{0}$}

Here we consider the dynamics near the point $P_{O}$ in system (6.13) by means of a spherical blow-up. We drop subscripts in (6.13), and define the blow-up by the transformation

$$
\sigma \geq 0,(\bar{x}, \bar{r}, \bar{\epsilon}) \in S^{2} \mapsto\left\{\begin{array}{l}
x=\sigma \bar{x}, \\
r=\sigma \bar{r}, \\
\epsilon=\sigma \bar{\epsilon} .
\end{array}\right.
$$

We are primarily interested in the dynamics observable in the phase directional charts

$$
\widetilde{\mathcal{K}}_{1}: \bar{r}=1, \quad \widetilde{\mathcal{K}}_{2}: \bar{\epsilon}=1,
$$

for which we introduce the chart specific coordinates

$$
\begin{aligned}
& \widetilde{\mathcal{K}}_{1}: x=\sigma_{1} x_{1}, \quad r=\sigma_{1}, \quad \epsilon=\sigma_{1} \epsilon_{1}, \\
& \widetilde{\mathcal{K}}_{2}: x=\sigma_{2} x_{2}, \quad r=\sigma_{2} r_{2}, \quad \epsilon=\sigma_{2} .
\end{aligned}
$$

We adopt the following notational convention: given an object $\gamma$ identified in chart $\mathcal{K}_{2}$, we denote its image under the blow-up transformation (6.15) by $\tilde{\gamma}$, and it's image in a particular coordinate chart $\tilde{\mathcal{K}}_{i}$ by $\tilde{\gamma}_{i}$. 
The transition map between charts $\widetilde{\mathcal{K}}_{1}$ and $\widetilde{\mathcal{K}}_{2}$ is given by

$$
\begin{array}{llll}
\tilde{\kappa}_{12}: x_{1}=r_{2}^{-1} x_{2}, & \epsilon_{1}=r_{2}^{-1}, & \sigma_{1}=\sigma_{2} r_{2}, & r_{2}>0, \\
\tilde{\kappa}_{21}: x_{2}=\epsilon_{1}^{-1} x_{1}, & r_{2}=\epsilon_{1}^{-1}, & \sigma_{2}=\sigma_{1} \epsilon_{1}, & \epsilon_{1}>0 .
\end{array}
$$

\section{$\widetilde{\mathcal{K}}_{1}$ Chart}

The dynamics in chart $\widetilde{\mathcal{K}}_{1}$ are governed by

$$
\begin{aligned}
& x_{1}^{\prime}=\epsilon_{1}\left(a+\sigma_{1} e^{-2\left(\sigma_{1} \epsilon_{1}\right)^{-1}}\right)+x_{1}\left(1+\sigma_{1} \epsilon_{1}\right)\left(x_{1}+b\left(1-2 e^{-\left(\sigma_{1} \epsilon_{1}\right)^{-1}}\right)\right), \\
& \epsilon_{1}^{\prime}=2 \epsilon_{1}\left(1+\sigma_{1} \epsilon_{1}\right)\left(x_{1}+b\left(1-2 e^{-\left(\sigma_{1} \epsilon_{1}\right)^{-1}}\right)\right), \\
& \sigma_{1}^{\prime}=-\sigma_{1}\left(2+\sigma_{1} \epsilon_{1}\right)\left(x_{1}+b\left(1-2 e^{-\left(\sigma_{1} \epsilon_{1}\right)^{-1}}\right)\right),
\end{aligned}
$$

after a suitable desingularization (division by $\sigma_{1}$ ). We identify an equilibrium for the system $(6.18)$ at $p_{r}:(0,0,0)$, as well as a line of equilibria

$$
\tilde{\mathcal{N}}_{1}=\left\{\left(-b, 0, \sigma_{1}\right): \sigma_{1} \geq 0\right\},
$$

corresponding to the extension of the line of equilbria $\mathcal{N}_{2}^{\prime}$ observed in chart $\mathcal{K}_{2}$. Note that $\widetilde{\mathcal{N}}_{1}$ terminates at $p_{l}:(-b, 0,0)$.

Lemma 6.5 The following hold for system (6.18):

(i) The point $p_{l}:(-b, 0,0)$ is partially hyperbolic with a single non-zero eigenvalue $\lambda=-b<0$. There exists a corresponding two-dimensional center manifold $\widetilde{M}_{1}$ tangent to the center eigenspace $E^{c}\left(p_{l}\right)$, which can be chosen to be the extension of the manifold $M_{1}^{\prime}$.

The manifold $\left.\tilde{M}_{1}\right|_{\epsilon_{1}=0}$ contains the one-dimensional manifold $\tilde{\mathcal{N}}_{1}$ as a normally hyperbolic and attracting manifold of equilbria. Moreover, $\left.\widetilde{M}_{1}\right|_{\sigma_{1}=0}$ contains a unique one-dimensional center manifold $\widetilde{\mathcal{W}}_{1}$, and the slow flow on $\widetilde{\mathcal{W}}_{1}$ is increasing in the $\epsilon_{1}$-coordinate.

(ii) The equilibrium $p_{r}:(0,0,0)$ is a hyperbolic saddle with eigenvalues

$$
\lambda=b, 2 b,-2 b .
$$

The stable manifold $W^{s}\left(p_{r}\right)$ is contained in $x_{1}=\epsilon_{1}=0$, which is invariant.

Proof The statement (i) follows after linearization of the system (6.18) and an application of the center manifold theorem. In particular, one obtains the following graph expression for $\widetilde{\mathcal{W}}_{1}$ via the usual matching approach:

$$
\tilde{\mathcal{W}}_{1}: x_{1}=-b+\frac{a}{1+b} \epsilon_{1}+\mathcal{O}\left(\epsilon_{1}^{2}\right) \text {. }
$$

Hence the dynamics on $\widetilde{\mathcal{W}}_{1}$ are governed by

$$
\begin{aligned}
x_{1}^{\prime} & =\frac{a \epsilon_{1}}{1+b}+\mathcal{O}\left(\epsilon_{1}^{2}\right), \\
\epsilon_{1}^{\prime} & =\frac{2 a \epsilon_{1}^{2}}{1+b}+\mathcal{O}\left(\epsilon_{1}^{3}\right),
\end{aligned}
$$

from which assertion (i) follows. 
The statement (ii) follows after linearization of the system (6.18), and the observation that restriction to $x_{1}=\epsilon_{1}=0$ gives

$$
\begin{aligned}
& x_{1}^{\prime}=0, \\
& \sigma_{1}^{\prime}=-2 b \sigma_{1} .
\end{aligned}
$$

\section{$\widetilde{\mathcal{K}}_{2}$ Chart}

The dynamics in chart $\widetilde{\mathcal{K}}_{2}$ are governed by

$$
\begin{aligned}
& x_{2}^{\prime}=r_{2}\left(a+\sigma_{2} r_{2} e^{-2 \sigma_{2}^{-1}}\right)-x_{2}\left(1+\sigma_{2}\right)\left(x_{2}+b r_{2}\left(1-2 e^{-\sigma_{2}^{-1}}\right)\right), \\
& r_{2}^{\prime}=-2 r_{2}\left(1+\sigma_{2}\right)\left(x_{2}+b r_{2}\left(1-2 e^{-\sigma_{2}^{-1}}\right)\right), \\
& \sigma_{2}^{\prime}=\sigma_{2}^{2}\left(x_{2}+b r_{2}\left(1-2 e^{-\sigma_{2}^{-1}}\right)\right),
\end{aligned}
$$

after a suitable desingularization (division by $\sigma_{2}$ ). The system (6.19) has a line of equilibria

$$
\widetilde{L}_{e, 2}=\{(0,0, \epsilon): \epsilon \geq 0\} .
$$

Lemma 6.6 The following hold for system (6.19):

(i) The unique one-dimensional center manifold $\widetilde{\mathcal{W}}_{2}=\tilde{\kappa}_{12}\left(\widetilde{\mathcal{W}}_{1}\right)$ is contained within $\sigma_{2}=0$, and forward asymptotic to the nonhyperbolic point $p_{o}:(0,0,0)$. In particular, $\mathcal{W}_{2}$ approaches $p_{o}$ tangent to the positive $x_{2}$-axis.

(ii) The line $\widetilde{L}_{e, 2}$ is non-hyperbolic, and coincides where domains overlap with the nonhyperbolic line $L_{e, 2}^{\prime}$ observed in the $\mathcal{K}_{2}^{\prime}$ chart (and hence with the non-hyperbolic line $L_{3}$ observed in chart $K_{3}$ ).

Proof In order to prove the assertion (i) we consider the system in the invariant plane $\sigma_{2}=0$ :

$$
\begin{aligned}
& x_{2}^{\prime}=a r_{2}-x_{2}\left(x_{2}+b r_{2}\right), \\
& r_{2}^{\prime}=-2 r_{2}\left(x_{2}+b r_{2}\right) .
\end{aligned}
$$

The system (6.21) has a single non-hyperbolic equilibrium $p_{o}$ at $(0,0)$. Moreover, the region

$$
\mathcal{V}=\left\{\left(x_{2}, r_{2}\right):-b^{-1} r_{2} \leq x_{2} \leq a b^{-1}, r_{2} \geq 0\right\}
$$

bounded by the $x_{2}$-axis, the $r_{2}$-nullcline $\left\{\left(-b^{-1} r_{2}, r_{2}\right): r_{2} \geq 0\right\}$, and the vertical asymptote $\left\{\left(a b^{-1}, r_{2}\right): r_{2} \geq 0\right\}$ in the $x_{2}$-nullcline, is forward invariant. In particular, the $x_{2}$-axis is invariant with dynamics

$$
x_{2}^{\prime}=-x_{2}^{2}
$$

so that $x_{2}^{\prime}<0$ for $x_{2} \neq 0$; see Fig. 19. Now define a compact subset $\tilde{\mathcal{V}} \subset \mathcal{V}$ by

$$
\tilde{\mathcal{V}}=\left\{\left(x_{2}, r_{2}\right) \in \mathcal{V}: r_{2} \leq c_{2}\right\},
$$

and choose $c_{2}>0$ sufficiently large so that by Lemma $6.5, \widetilde{\mathcal{W}}_{2}=\kappa_{12}\left(\widetilde{\mathcal{W}}_{1}\right)$ enters $\widetilde{\mathcal{V}}$ transversally through $r_{2}=c_{2}$. Since $\tilde{\mathcal{V}}$ is compact and forward invariant, the PoincaréBendixon theorem applies, and $\widetilde{\mathcal{W}}_{2}$ is forward asymptotic to $p_{o}$ at $(0,0)$. 


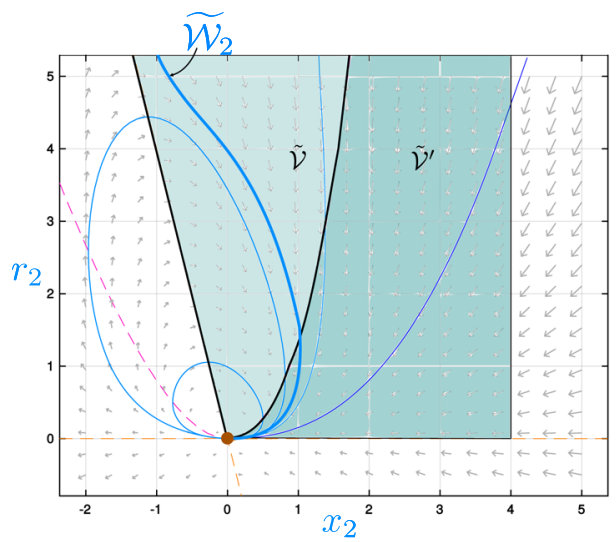

(a)

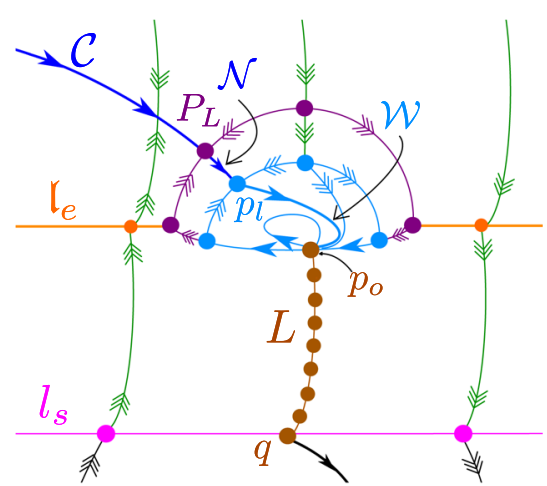

(b)

Fig. 19 Blow-up of the degenerate equilibrium $P_{O}$ (cyan) from Fig. 18 to a sphere (cyan). On the sphere the equilibrium $p_{l}$ is connected to the degenerate equilibrium $p_{0}$ (brown) by the manifold (heteroclinic orbit) $\mathcal{W}$ (cyan). In a The phase plane $\sigma_{2}=0$ in chart $\widetilde{\mathcal{K}}_{2}$ used in the proof of Lemma 6.6 regarding the asymptotic properties of the local version $\widetilde{\mathcal{W}}_{2}$ of $\mathcal{W}$ in chart $\widetilde{\mathcal{K}}_{2}$. The plane $\sigma_{2}=0$ covers the sphere $\sigma=0$ viewed from $\bar{\epsilon}=1$. In $\mathbf{b}$ A global picture for comparison (Color figure online)

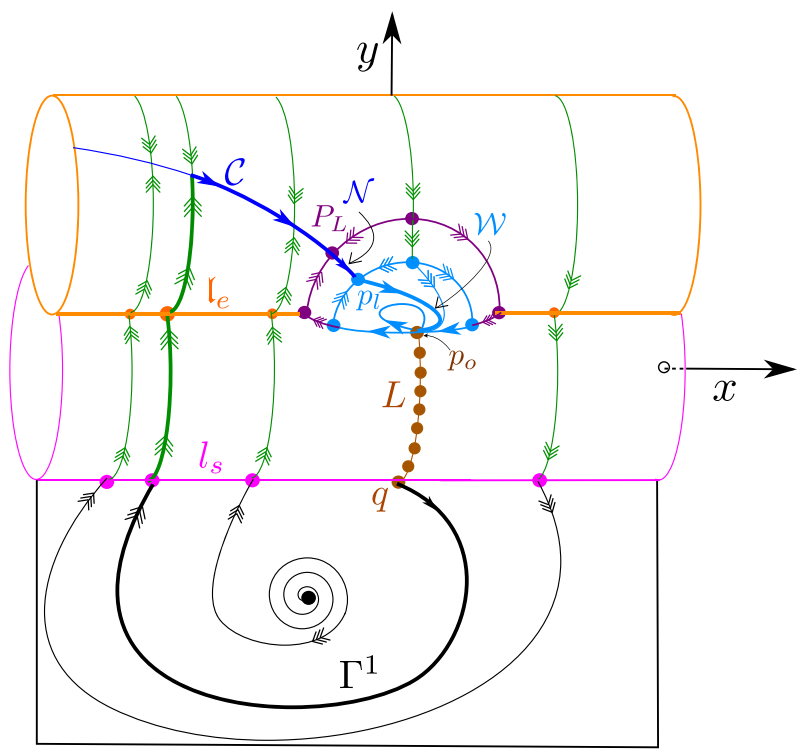

Fig. 20 Results after two spherical blow-ups: outer sphere (purple), inner sphere (cyan) (Color figure online)

To see that $\widetilde{\mathcal{W}_{2}}$ approaches $(0,0)$ tangent to the positive $x_{2}$-axis, notice that trajectories in $\tilde{\mathcal{V}} \backslash\{(0,0)\}$ reach the forward invariant region

$$
\tilde{\mathcal{V}}^{\prime}=\left\{\left(x_{2}, r_{2}\right) \in \tilde{\mathcal{V}}: r_{2} \leq \frac{x_{2}^{2}}{a-b x_{2}}\right\}
$$


bounded above by the component of the $x_{2}$-nullcline in the positive quadrant in finite time. Hence $\widetilde{\mathcal{V}}_{2}$ approaches $(0,0)$ from within $\widetilde{\mathcal{V}}^{\prime}$, and therefore tangent to the positive $x_{2}$-axis.

Statement (ii) is a straightforward calculation and application of the (successive) blowdown transformations.

The situation is sketched in Fig. 20.

\subsubsection{Blow-Up of $\tilde{L}_{e, 2}$}

Finally, in this subsection, we consider a cylindrical blow-up of the non-hyperbolic line $\widetilde{L}_{e, 2}$ identified in equation (6.20). This is done in a neighborhood of the point $p_{0}$ covered by chart $\widetilde{\mathcal{K}}_{2}$. In Sect. 6.2.1 we will carry out a similar cylindrical blow-up of the line $L$ within the first cylindrical blow-up as defined in (3.5), which needs to be carried out in coordinate charts $K_{i}, i=1,2,3$ defined in (3.6), (3.7), and (3.8). There, we will also show that these two cylindrical blow-ups match up.

We start with system (6.19), drop the subscripts, introduce a hat notation (needed in the process of matching the results obtained here with the results obtained in Sect. 6.2.1), and define a weighted blow-up transformation by the map

$$
\hat{s} \geq 0,(\overline{\hat{x}}, \overline{\hat{r}}) \in S^{1} \mapsto\left\{\begin{array}{l}
\hat{x}=\hat{s} \overline{\hat{x}}, \\
\hat{r}=\hat{s}^{2} \hat{\hat{r}} .
\end{array}\right.
$$

We are primarily interested in the dynamics observable in coordinate charts

$$
\widehat{K}_{31}: \overline{\hat{x}}=1, \quad \widehat{K}_{32}: \overline{\hat{r}}=1,
$$

for which we introduce chart specific coordinates

$$
\begin{array}{ll}
\widehat{K}_{31}: \hat{x}=\hat{s}_{1}, & \hat{r}=\hat{s}_{1}^{2} \hat{r}_{1}, \\
\widehat{K}_{32}: \hat{x}=\hat{s}_{2} \hat{x}_{2}, & \hat{r}=\hat{s}_{2}^{2} .
\end{array}
$$

The transition map between charts $\widehat{K}_{31}$ and $\widehat{K}_{32}$ is given by

$$
\begin{array}{lll}
\hat{\kappa}_{3132}: \hat{s}_{1}=\hat{s}_{2} \hat{x}_{2}, & \hat{r}_{1}=\hat{x}_{2}^{-2}, & \hat{x}_{2}>0, \\
\hat{\kappa}_{3231}: \hat{s}_{2}=\hat{s}_{1} \hat{r}_{1}^{1 / 2}, & \hat{x}_{2}=\hat{r}_{1}^{-1 / 2}, & \hat{r}_{1}>0 .
\end{array}
$$

The subscript notation, although a little cumbersome, will be helpful in when considering the dynamics in coordinate charts covering the lower portion of the blown-up line (circle) $L$.

\section{$\widehat{K}_{31}$ Chart}

The equations in the $\widehat{K}_{31}$ chart are given by

$$
\begin{aligned}
& \hat{r}_{1}^{\prime}=-2 \hat{r}_{1}^{2}\left(a+\sigma \hat{r}_{1} \hat{s}_{1}^{2} e^{-2 \sigma^{-1}}\right), \\
& \sigma^{\prime}=\sigma^{2}\left(1+b \hat{r}_{1} \hat{s}_{1}\left(1-2 e^{-\sigma^{-1}}\right)\right), \\
& \hat{s}_{1}^{\prime}=-\hat{s}_{1}\left(-\hat{r}_{1}\left(a+\sigma \hat{r}_{1} \hat{s}_{1}^{2} e^{-2 \sigma^{-1}}\right)+(1+\sigma)\left(1+b \hat{r}_{1} \hat{s}_{1}\left(1-2 e^{-\sigma^{-1}}\right)\right)\right),
\end{aligned}
$$

after a suitable desingularization (division by $\hat{s}_{1}$ ). The system (6.24) has a single equilibrium at $p_{s}:(0,0,0)$. 
Lemma 6.7 The following holds for system (6.24):

(i) The equilibrium $p_{s}$ is partially hyperbolic with a single nonzero eigenvalue $\lambda=-1$ and a corresponding two-dimensional local center manifold $\widehat{M}_{s}$ given by $\hat{s}_{1}=0$. The variable $\sigma$ is increasing along $\widehat{M}_{S} \cap\{\sigma>0\}$ while $\hat{r}_{1}$ is decreasing along $\widehat{M}_{S} \cap\left\{\hat{r}_{1}>0\right\}$.

(ii) The strong stable manifold $W^{s}\left(p_{s}\right)$ lies within $\hat{r}_{1}=\sigma=0$, and the $\hat{r}_{1}-\sigma-, \hat{s}_{1}$-axes are all invariant. In particular, $\hat{r}_{1}$ is decreasing along the $\hat{r}_{1}$-axis, $\sigma$ is increasing along the $\sigma$-axis (which we denote by $\mathcal{H}$ ), and $\hat{s}_{1}$ is decreasing along the $\hat{s}_{1}$-axis. Hence, $p_{s}$ is a non-hyperbolic saddle.

Proof The statement (i) follows after linearization at $p_{s}$, and an application of the center manifold theorem.

Invariance of the $\hat{r}_{1}-, \sigma-, \hat{s}_{1}$-axes follows immediately from the form taken by the equations when restricted to the respective axes. In $\left\{\hat{s}_{1}=\hat{r}_{1}=0\right\}$ we have

$$
\sigma^{\prime}=\sigma^{2}
$$

in $\left\{\hat{s}_{1}=\sigma=0\right\}$ we have

$$
\hat{r}_{1}^{\prime}=-2 a \hat{r}_{1}^{2}
$$

and in $\left\{\sigma=\hat{r}_{1}=0\right\}$ we have

$$
\hat{s}_{1}^{\prime}=-\hat{s}_{1}
$$

The assertion (ii) follows.

\section{$\widehat{K}_{32}$ Chart}

We omit the details in chart $\widehat{K}_{32}$ for the sake of brevity, simply noting that calculations reveal no equilibria and an invariant flow along the 'equator' $\hat{s}_{2}=\sigma=0$, as indicated in Fig. 21.

\subsection{Blow-Up of $L$ in the Algebraic Regime}

In order to obtain the fully nondegenerate singular cycle, it remains to blow-up the vertical non-hyperbolic line (circle) $L$ in the algebraic regime. We return to the dynamics observable after the (first) cylindrical blow-up, as defined in (3.5), i.e. the problem considered in coordinate charts $K_{i}, i=1,2,3$. In Sect. 6.2.1 we introduce the blow-up of $L$, and show that the dynamics observed in Sect. 6.1.4 can be related to the dynamics observed in this blow-up in an overlapping domain. In Sect. 6.2.2 we derive the qualitative properties of the dynamics associated with the 'lower part' of the vertical cylinder in Fig. 14.

\subsubsection{Blow-Up of $L$}

We consider the dynamics near the lower portion of the non-hyperbolic line (circle) $L$. We introduce a secondary weighted blow-up defined via the transformation

$$
(x, r,(\bar{y}, \bar{\epsilon})) \mapsto(s,(\bar{x}, \bar{r}),(\bar{y}, \bar{\epsilon})),
$$

where

$$
s \geq 0,(\bar{x}, \bar{r}) \in S^{1} \mapsto\left\{\begin{array}{l}
x=s \bar{x}, \\
r=s^{2} \bar{r}
\end{array}\right.
$$




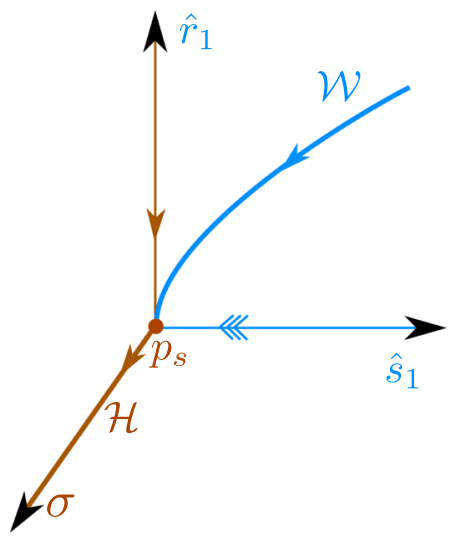

(a)

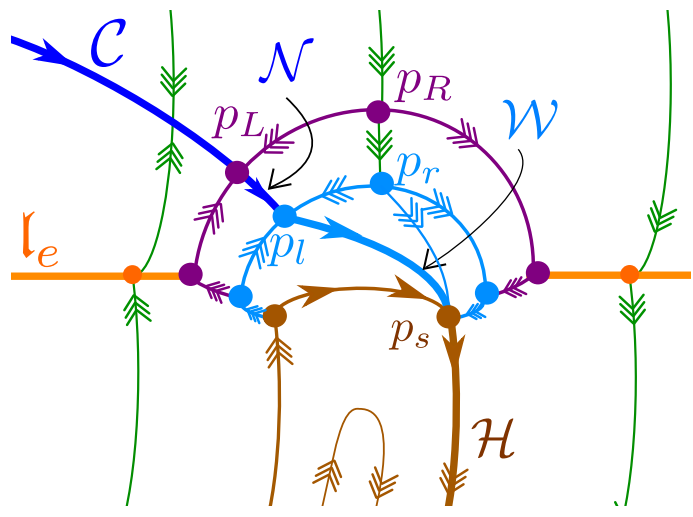

(b)

Fig. 21 Blow-up of the line of equilibria $L_{e}$ (brown) to a cylinder (brown). In a Local picture near the hyperbolic equilibrium $p_{s}$ in chart $\widehat{K}_{31}$. The cylinder corresponds to the plane $\hat{s}_{1}=0$ (brown). The plane $\sigma=0$ corresponds to the (inner) sphere (cyan). By the cylindrical blowup of $L_{e}$, we have gained hyperbolicity. The line $\mathcal{H}$ (brown) is the (non-hyperbolic) unstable manifold of $p_{s}$ (also brown). In $\mathbf{b}$ A global picture for comparison (Color figure online)

Composing this with the map (3.5), we obtain

$$
s \geq 0,(\bar{y}, \bar{\epsilon}) \in S^{1},(\bar{x}, \bar{r}) \in S^{1} \mapsto\left\{\begin{array}{l}
x=s \bar{x}, \\
y=s^{2} \bar{r} \bar{y}, \\
\epsilon=s^{2} \bar{r} \bar{\epsilon} .
\end{array}\right.
$$

Geometrically, the transformation (6.26) blows up the circle of non-hyperbolic points $L$ to the torus $\{s=0\} \times S^{1} \times S^{1}$, for which only the subset defined by $\bar{r} \geq 0$ and $\bar{\epsilon} \geq 0$ is relevant. In total, six coordinate charts are necessary for an understanding of the main dynamical features:

$$
\begin{array}{lll}
K_{11}: \bar{y}=-1, \bar{x}=1, & K_{21}: \bar{\epsilon}=1, \bar{x}=1, & K_{31}: \bar{y}=1, \bar{x}=1, \\
K_{12}: \bar{y}=-1, \bar{r}=1, & K_{22}: \bar{\epsilon}=1, \bar{r}=1, & K_{32}: \bar{y}=1, \bar{r}=1 .
\end{array}
$$

In particular $\bar{x}=1$ in charts $K_{i 1}$ and $\bar{r}=1$ in charts $K_{i 2}$, for $i=1,2,3$, and the subscript $i$ signifies the 'visible region' of the first (horizontal) cylinder defined by the blow-up transformation (3.5). For charts $K_{1 j}$ covering the region visible in $\bar{y}=-1$ we have chart specific coordinates

$$
\begin{array}{lll}
K_{11}: x=s_{1}, & y=-s_{1}^{2} r_{11}, & \epsilon=s_{1}^{2} r_{11} \epsilon_{1}, \\
K_{12}: x=s_{2} x_{2}, & y=-s_{2}^{2}, & \epsilon=s_{2}^{2} \epsilon_{1} .
\end{array}
$$

For charts $K_{2 j}$ covering the region visible in $\bar{\epsilon}=1$ we have chart specific coordinates

$$
\begin{array}{lll}
K_{21}: x=s_{1}, & y=s_{1}^{2} r_{21} y_{2}, & \epsilon=s_{1}^{2} r_{21}, \\
K_{22}: x=s_{2} x_{2}, & y=s_{2}^{2} y_{2}, & \epsilon=s_{2}^{2} .
\end{array}
$$


For charts $K_{3 j}$ covering the region visible in $\bar{y}=1$ we have chart specific coordinates

$$
\begin{aligned}
& K_{31}: x=s_{1}, \quad y=s_{1}^{2} r_{31}, \quad \epsilon=s_{1}^{2} r_{31} \epsilon_{3}, \\
& K_{32}: x=s_{2} x_{2}, \quad y=s_{2}^{2}, \quad \epsilon=s_{2}^{2} \epsilon_{3} \text {. }
\end{aligned}
$$

The transition maps between overlapping charts are given by

$$
\begin{aligned}
& \kappa_{1112}: s_{1}=s_{2} x_{2}, \quad r_{11}=x_{2}^{-2}, \quad x_{2}>0, \\
& \kappa_{1121}: r_{11}=-r_{21} y_{2}, \quad \epsilon_{1}=-y_{2}^{-1}, \quad y_{2}<0, \\
& \kappa_{1122}: s_{1}=s_{2} x_{2}, \quad r_{11}=-x_{2}^{-2} y_{2}, \quad \epsilon_{1}=-y_{2}^{-1}, \quad x_{2}>0, y_{2}<0 \text {, } \\
& \kappa_{1221}: s_{2}=\left(-r_{21} y_{2}\right)^{1 / 2}, \quad \epsilon_{1}=-y_{2}^{-1}, \quad x_{2}=\left(-r_{21} y_{2}\right)^{-1 / 2}, \quad y_{2}<0, r_{21}>0 \text {, } \\
& \kappa_{2122}: s_{1}=s_{2} x_{2}, \quad r_{21}=x_{2}^{-2}, \quad x_{2}>0, \\
& \kappa_{2131}: r_{21}=r_{31} \epsilon_{3}, \quad y_{2}=\epsilon_{3}^{-1}, \quad \epsilon_{3}>0, \\
& \kappa_{2132}: s_{1}=s_{2} x_{2}, \quad r_{21}=x_{2}^{-2} \epsilon_{3}, \quad y_{2}=\epsilon_{3}^{-1}, \quad x_{2}, \epsilon_{3}>0, \\
& \kappa_{2231}: s_{2}=s_{1}\left(r_{31} \epsilon_{3}\right)^{1 / 2}, \quad x_{2}=s_{1}\left(r_{31} \epsilon_{3}\right)^{-1 / 2}, \quad y_{2}=\epsilon_{3}^{-1}, \quad r_{31}, \epsilon_{3}>0 \text {, } \\
& \kappa_{3132}: s_{1}=s_{2} x_{2}, \quad r_{21}=x_{2}^{-2}, \quad x_{2}>0,
\end{aligned}
$$

and their inverses can be computed directly using these expressions if necessary.

We will focus in this section on the dynamics observable in charts $K_{i j}, i=1,2, j=1,2$. The dynamics in charts $K_{i j}$ with $i=3$ have already been considered in Sect. 6.1.4, as is shown in the following result.

Lemma 6.8 Coordinates $\left(r_{31}, \epsilon_{3}, s_{1}\right)$ in chart $K_{31}$ are related to coordinates $\left(\hat{r}_{1}, \sigma, \hat{s}_{1}\right)$ in chart $\widehat{K}_{31}$ via

$$
r_{31}=\sigma^{-1} \hat{r}_{1}, \quad \epsilon_{3}=\sigma e^{\sigma^{-1}}, \quad s_{1}=\sigma e^{-\sigma^{-1}} \hat{s}_{1}, \quad \sigma>0 .
$$

Coordinates $\left(x_{2}, \epsilon_{3}, s_{2}\right)$ in chart $K_{32}$ are related to coordinates $\left(\hat{x}_{2}, \sigma, \hat{s}_{2}\right)$ in chart $\widehat{K}_{32}$ via

$$
x_{2}=\sigma^{1 / 2} e^{-(2 \sigma)^{-1}} \hat{x}_{2}, \quad \epsilon_{3}=\sigma, \quad s_{2}=\sigma^{1 / 2} e^{-(2 \sigma)^{-1}} \hat{s}_{2}, \quad \sigma>0 .
$$

Proof The expressions given for the coordinates $\left(r_{31}, \epsilon_{3}, s_{1}\right)$ in chart $K_{31}$ are obtained by composing blow-up maps (3.8), (3.21), (6.16) and the coordinates for $\widehat{K}_{31}$ in (6.23). In the notation below, we avoid dropping subscripts and append them at each coordinate change (except where the 'hat notation' suffices). Explicitly, the first three compositions give

$$
\begin{aligned}
& x=v_{2} x_{2}, \\
& y=r_{3}=\rho_{1} r_{31}=v_{2}^{2} r_{312}, \\
& \epsilon=r_{3} \epsilon_{3}=v_{2} r_{312} \epsilon_{3}, \\
& q=\rho_{1}=v_{2},
\end{aligned}
$$

and subsequent restriction to $\nu_{2}=e^{-\epsilon_{3}^{-1}}$ gives

$$
x=e^{-\epsilon_{3}^{-1}} x_{2}, \quad y=e^{-2 \epsilon_{3}^{-1}} r_{312}, \quad \epsilon=e^{-\epsilon_{3}^{-1}} r_{312} \epsilon_{3} .
$$


The last two compositions give

$$
\begin{aligned}
& x=e^{-\epsilon_{3}^{-1}} x_{2}=e^{-\sigma_{2}^{-1}} x_{2} \sigma_{2}=\sigma_{2} e^{-\sigma_{2}^{-1}} \hat{s}_{1}, \\
& y=e^{-2 \epsilon_{3}^{-1}} r_{312}=e^{-2 \sigma_{2}^{-1}} \sigma_{2} r_{312}=\sigma_{2} \hat{r}_{1} e^{-2 \sigma_{2}^{-1}} \hat{s}_{1}^{2} \text {, } \\
& \epsilon=e^{-\epsilon_{3}^{-1}} r_{312} \epsilon_{3}=e^{-\sigma_{2}^{-1}} \sigma_{2}^{2} r_{312}=\sigma_{2}^{2} \hat{r}_{1} e^{-\sigma_{2}^{-1}} \hat{s}_{1}^{2} \text {. }
\end{aligned}
$$

Dropping the subscript in $\sigma_{2}$ and comparing with the $K_{31}$ coordinates given in (6.29) yields the result.

The expressions given for the coordinates $\left(x_{2}, \epsilon_{3}, s_{2}\right)$ in chart $K_{32}$ are obtained by a similar argument: composing blow-up maps (3.8), (3.21), (6.16) and the coordinates for $\widehat{K}_{32}$ in (6.23) gives

$$
x=\sigma \hat{x}_{2} e^{-\sigma^{-1}} \hat{s}_{2}, \quad y=\sigma e^{-2 \sigma^{-1}} \hat{s}_{2}^{2}, \quad \epsilon=\sigma^{2} e^{-\sigma^{-1}} \hat{s}_{2}^{2},
$$

(where we have dropped the subscript in $\sigma_{2}$ ), and direct comparison with the expression for $K_{32}$ coordinates in (6.29) yields the desired result.

We obtain the following corollary.

Corollary 6.9 The (invariant) $\sigma$-axis in system (6.24) is mapped to

$$
\mathcal{H}_{31}=\left\{\left(0, \epsilon_{3}, 0\right): \epsilon_{3}>0\right\}
$$

under the transformation defined by the equations (6.30), which is invariant for the system obtained in chart $K_{31}$ coordinates. Dynamics on $\mathcal{H}_{31}$ are governed by

$$
\epsilon_{3}^{\prime}=\epsilon_{3} e^{-\epsilon_{3}^{-1}}
$$

Proof This is an immediate consequence of Lemma 6.8 and the form of the equations obtained in chart $K_{31}$, which are given by

$$
\begin{aligned}
r_{31}^{\prime} & =-r_{31}\left(b r_{31} s_{1}+e^{-\epsilon_{3}^{-1}}\left(1-2 b r_{31} s_{1}+2 r_{31} a+2 r_{31} s_{1}^{2}\right)\right), \\
\epsilon_{3}^{\prime} & =\epsilon_{3}\left(b r_{31} s_{1}+e^{-\epsilon_{3}^{-1}}\left(1-2 b r_{31} s_{1}\right)\right), \\
s_{1}^{\prime} & =s_{1} r_{31} e^{-\epsilon_{3}^{-1}}\left(a+s_{1}^{2} r_{31}\right),
\end{aligned}
$$

after a suitable desingularization (division by $s_{1}$ ). The expression in (6.31) follows by restriction to $r_{31}=s_{1}=0$.

\subsubsection{Blow-Up for the Lower Part of $L$}

Lemma 6.8 shows how the dynamics in the transitional regime can be related to the dynamics in the algebraic regime, after application of the blow-up transformation (6.26). Moreover, Lemma 6.7 and Corollary 6.9 are sufficient for an understanding of the main dynamical features and in particular, the construction of $\Gamma_{0}$. Hence, we restrict attention here to dynamics in charts $K_{11}, K_{12}, K_{21}, K_{22}$ only in this section. 


\subsection{3 $K_{22}$ Chart}

We can determine the equations in the chart $K_{22}$ by considering the system (3.2) on the fast time scale with $\epsilon=r_{2}$, i.e.

$$
\begin{aligned}
& x^{\prime}=r_{2}^{2}\left(a+r_{2} y\right), \\
& y_{2}^{\prime}=-x+b r_{2} y_{2}\left(2-e^{y_{2}}\right), \quad r_{2} \ll 1,
\end{aligned}
$$

and then apply the secondary transformation defined by the equations

$$
\begin{aligned}
& x=s_{2} x_{2}, \\
& r=s_{2}^{2} .
\end{aligned}
$$

This produces the following system

$$
\begin{aligned}
& x_{2}^{\prime}=a+s_{2}^{2} y_{2}, \\
& y_{2}^{\prime}=-x_{2}+b s_{2} y_{2}\left(2-e^{y_{2}}\right), \quad s_{2} \ll 1,
\end{aligned}
$$

after a suitable desingularization (division by $s_{2}$ ).

Lemma 6.10 The system (6.34) is a regular perturbation problem, with leading order dynamics on compact domains determined by the dynamics of the limiting system

$$
\begin{aligned}
& x_{2}^{\prime}=a, \\
& y_{2}^{\prime}=-x_{2},
\end{aligned}
$$

for which all orbits are of the form

$$
y_{2}\left(x_{2}\right)=-\frac{x_{2}^{2}}{2 a}+c_{0},
$$

for constants $c_{0}$.

Proof This follows by direct integration of the equations (6.35).

\subsection{4 $K_{21}$ Chart}

The equations in chart $K_{21}$ can be determined by considering the system (6.32) and applying the secondary transformation defined by the equations

$$
\begin{aligned}
x & =s_{1}, \\
r_{2} & =s_{1}^{2} r_{21} .
\end{aligned}
$$

We obtain the following system,

$$
\begin{aligned}
y_{2}^{\prime} & =-1+b s_{1} r_{21} y_{2}\left(2-e^{y_{2}}\right), \\
r_{21}^{\prime} & =-2 s_{1}^{2} r_{21}^{3}\left(a-s_{1}^{2} r_{21} y_{2}\right), \\
s_{1}^{\prime} & =s_{1}^{3} r_{21}^{2}\left(a-s_{1}^{2} r_{21} y_{2}\right),
\end{aligned}
$$

after applying a time desingularization (division by $s_{1}$ ). 
Lemma 6.11 The system (6.36) is invariant in subspaces $r_{21}=0, s_{1}=0$, and along the invariant line

$$
\mathcal{H}_{21}=\left\{\left(y_{2}, 0,0\right): y_{2} \in \mathbb{R}\right\} .
$$

In all three subspaces $y_{2}$ is the only dynamic variable, with dynamics governed by

$$
y_{2}^{\prime}=-1 \text {. }
$$

Proof Straightforward restriction.

\subsection{5 $K_{12}$ Chart}

The equations in the chart $K_{12}$ can be determined by considering the system (3.11) and applying the secondary transformation

$$
x=s_{2} x_{2}, \quad r_{1}=s_{2}^{2} .
$$

One obtains the system

$$
\begin{aligned}
& x_{2}^{\prime}=a-s_{2}^{2}-\frac{1}{2} x_{2}\left(x_{2}+b s_{2}\left(2-e^{-\epsilon_{1}^{-1}}\right)\right), \\
& \epsilon_{1}^{\prime}=-\epsilon_{1}\left(x_{2}+b s_{2}\left(2-e^{-\epsilon_{1}^{-1}}\right)\right), \\
& s_{2}^{\prime}=\frac{1}{2} s_{2}\left(x_{2}+b s_{2}\left(2-e^{-\epsilon_{1}^{-1}}\right)\right),
\end{aligned}
$$

after a suitable desingularization (division by $s_{2}$ ). The system (6.38) has three equilibria:

$$
q_{i}=(-\sqrt{2 a}, 0,0), \quad p_{12}=(-2 b \sqrt{a}, \sqrt{a}, 0), \quad q_{o}=(\sqrt{2 a}, 0,0) .
$$

Lemma 6.12 The following holds for the system (6.38):

(i) The equilbria $q_{i}$ and $q_{o}$ are hyperbolic saddles with eigenvalues

$$
\lambda_{1, i}=\sqrt{\frac{a}{2}}, \quad \lambda_{2, i}=\sqrt{2 a}, \quad \lambda_{3, i}=-\sqrt{\frac{a}{2}},
$$

and

$$
\lambda_{1, o}=-\sqrt{\frac{a}{2}}, \quad \lambda_{2, o}=-\sqrt{2 a}, \quad \lambda_{3, o}=\sqrt{\frac{a}{2}},
$$

respectively. There is a strong resonance in each case due to the relation $\lambda_{1, i / o}=\lambda_{2, i / o}+$ $\lambda_{3, i / o}$

(ii) The equilibrium $p_{12}$ is an unstable focus within the invariant $\epsilon_{1}=0$ plane for any $b>0$, $a \in(0,2)$, and coincides upon coordinate change with the true equilibrium of the system $p$.

(iii) The lines

$$
\mathcal{G}_{ \pm, 12}=\left\{\left( \pm \sqrt{2 a}, \epsilon_{1}, 0\right): \epsilon_{1} \geq 0\right\}
$$

are invariant. 
Proof Statements (i) and (ii) are immediate upon linearization of the system (6.38) and an application of the blow-down map, respectively. To prove the statement (iii), consider the system in the invariant plane $s_{2}=0$ :

$$
\begin{aligned}
& x_{2}^{\prime}=a-\frac{1}{2} x_{2}^{2}, \\
& \epsilon_{1}^{\prime}=-\epsilon_{1} x_{2} .
\end{aligned}
$$

It is easy to verify that this system has invariant lines along $x_{2}= \pm \sqrt{2 a}, \epsilon_{1} \geq 0$.

\subsection{6 $K_{11}$ Chart}

The equations in the chart $K_{11}$ can be determined by considering the system (3.11) and applying the secondary transformation

$$
x=s_{1}, \quad r_{1}=s_{1}^{2} r_{11} .
$$

One obtains the system

$$
\begin{aligned}
r_{11}^{\prime} & =r_{11}\left(1+b s_{1} r_{11}\left(2-e^{-\epsilon_{1}^{-1}}\right)-2 r_{11}\left(a-r_{11} s_{1}^{2}\right)\right), \\
\epsilon_{1}^{\prime} & =-\epsilon_{1}\left(1+b s_{1} r_{11}\left(2-e^{-\epsilon_{1}^{-1}}\right)\right), \\
s_{1}^{\prime} & =s_{1} r_{11}\left(a-r_{11} s_{1}^{2}\right),
\end{aligned}
$$

after a time desingularization (division by $s_{1}$ ), for which there are two equilibria:

$$
q_{s}=(0,0,0), \quad q_{o, 2}=\left(0, \frac{1}{2 a}, 0\right) .
$$

Lemma 6.13 The following holds for the system (6.41):

(i) The equilibrium $q_{s}$ is partially hyperbolic with a eigenvalues

$$
\lambda=1,-1,0 .
$$

The equilibrium $q_{o, 2}$ coincides with the hyperbolic saddle $q_{o}$ observed in chart $K_{12}$.

(ii) The lines

$$
\mathcal{G}_{+, 11}=\left\{\left(\frac{1}{2 a}, \epsilon_{1}, 0\right): \epsilon_{1} \geq 0\right\}, \quad \mathcal{H}_{11}=\left\{\left(0, \epsilon_{1}, 0\right): \epsilon_{1} \geq 0\right\},
$$

are invariant, with $\epsilon_{1}$ decreasing along $\mathcal{G}_{11}$, and decreasing along $\mathcal{H}_{11}$.

Proof The statement (i) follows immediately by a linearization of (6.41) and an application of the transition map $\kappa_{1112}$.

To prove the statement (ii), consider the system in the invariant plane $s_{1}=0$ :

$$
\begin{aligned}
r_{11}^{\prime} & =r_{11}\left(1-2 a r_{11}\right), \\
\epsilon_{1}^{\prime} & =-\epsilon_{1} .
\end{aligned}
$$

The equations decouple, and the lines along $r_{11}=1 / 2 a, \epsilon_{1} \geq 0$ and $r_{11}=0, \epsilon_{1} \geq 0$ are invariant with dynamics governed in each case by

$$
\epsilon_{1}^{\prime}=-\epsilon_{1} \text {. }
$$

Hence $\epsilon_{1}$ is decreasing along $\mathcal{G}_{11}$, and decreasing along $\mathcal{H}_{11}$. 


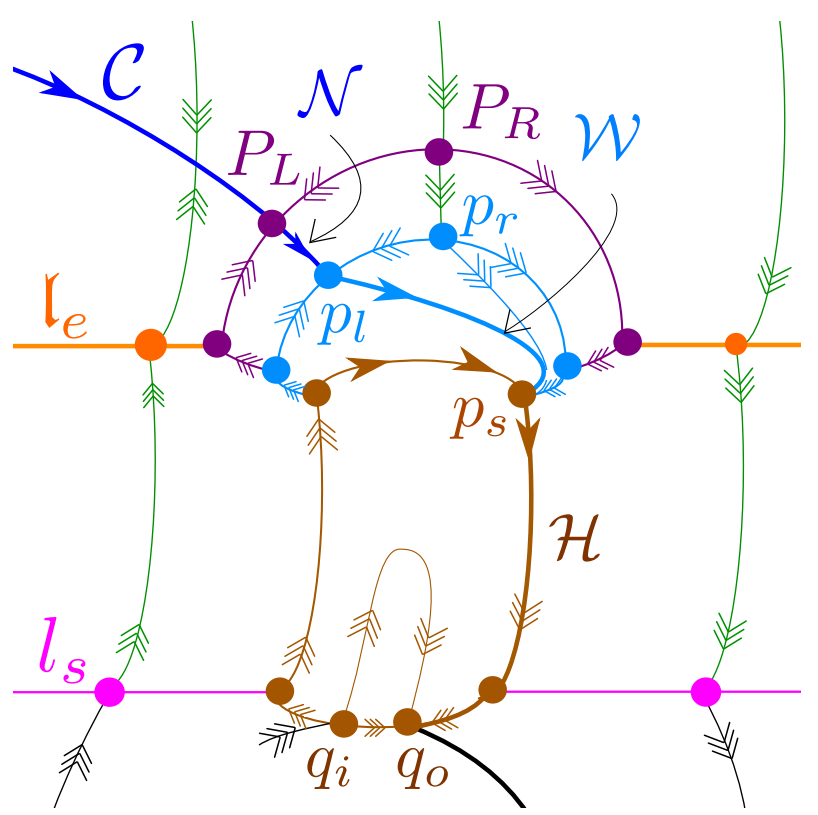

Fig. 22 The complete desingularization near the line $L$ of degenerate equilibria

Taken together, Lemma 6.10, Lemma 6.11, Lemma 6.12 and Lemma 6.13 imply the main dynamical features associated with the lower portion of the blow-up of $L$, as sketched in Fig. 22. Note the resemblance to the regular fold when viewed 'from below' (as one might expect due to the presence of the quadratic tangency in the PWS system). For further details on the regular fold see [22]. This resemblance is further exemplified by the fact that the invariant lines $\mathcal{G}_{ \pm}$observed in the $K_{1 j}$ charts connect in the region of the cylinder visible in the $K_{2 j}$ charts in Fig. 22; this is shown below.

Proposition 6.14 The invariant lines $\mathcal{G}_{ \pm}$observed in charts $K_{1 j}$ connect along the invariant parabola given in chart $K_{22}$ coordinates by

$$
y_{2}\left(x_{2}\right)=-\frac{x_{2}^{2}}{2 a}, r_{2}=0
$$

Proof Starting from chart $K_{11}$ and applying the relevant blow-down transformation, we may parameterize the $\mathcal{G}_{+}$in chart $K_{1}$ coordinates as

$$
\mathcal{G}_{+, 1}=\left\{\left(x, \frac{x^{2}}{2 a}, \epsilon_{1}\right): \epsilon_{1} \geq 0\right\}
$$

Applying the $\kappa_{12}$ transition map in (3.9) gives the following parameterization for $\mathcal{G}_{+}$in chart $K_{2}$,

$$
\mathcal{G}_{+, 2}=\left\{\left(x, y_{2},-\frac{x^{2}}{2 a y_{2}}\right): y_{2} \leq 0\right\}
$$


and applying the secondary blow-up transformation (6.33) and expressing $\mathcal{G}_{+, 2}$ in chart $K_{22}$ coordinates gives

$$
r_{2}=s_{2}^{2}=-\frac{x^{2}}{2 a y_{2}}=-\frac{s_{2}^{2} x_{2}^{2}}{2 a y_{2}} \quad \Longrightarrow \quad y_{2}\left(x_{2}\right)=-\frac{x_{2}^{2}}{2 a} .
$$

A similar argument applies for $\mathcal{G}_{-}$, and the result follows.

\section{Summary and Outlook}

In this paper, we have proved existence of limit cycles in two prototypical examples with singular exponential nonlinearities. Our approach was geometric and consisted of the following: Firstly, we applied a proper normalization by dividing the right hand side by a suitable factor, producing a PWS limit as $\epsilon \rightarrow 0$. Secondly, we applied a modification of the blow-up approach following e.g. [22,23] to deal with degeneracies of this type, recall Sect. 2.2. For the Hester system, this basically led to a system with desirable hyperbolicity properties allowing us to perturb away from a singular cycle. Under the assumptions (1.11), the exponential nonlinearities did therefore not provide any obstacles for this result. For the Le Corbeiller problem, we did not recover the essential geometric structure, recall Fig. 7, by a simple scaling. Instead the 'slow manifold' was hidden within a separate 'exponential' blow-up, see $\mathcal{C}$ in Fig. 11. This also led to more complicated asymptotics, recall Lemma 1.6, which we were able to capture using the method in [20]. The manner in which the analyses differed for Hester and Le Corbeiller oscillators is also reflected dynamically. For example, the relaxation oscillations in the Hester system are genuinely slow-fast in the sense that they exhibit a long period of inactivity, followed by a rapid active phase, the reset; recall Remark 2.1. Hence, the period of these oscillations is approximated to leading order by the amount of time spent in the vicinity of the attracting slow manifold, and tends to infinity as $\epsilon \rightarrow 0$. By contrast, the leading order dynamics along the invariant manifold corresponding to the inactive phase of the Le Corbeiller relaxation oscillations is governed by $\dot{x}=a$; recall Lemma 1.6. Here, both active and inactive phases of the relaxation oscillations occur on the same timescale. Thus the leading order approximation of the period is determined by the time taken to flow along the orbit segment $\Gamma^{1}$ according to (1.17), plus the time taken to flow along $\Gamma^{2}$ according to $\dot{x}=a$.

There are a number of directions in which one may extend the analysis of the prototypical Hester and Le Corbeiller oscillators considered herein. For example, assume in the Hester oscillator case that $(1.11)$ is violated by $\gamma \geq 1$; then $(0,0)$ is a stable node for $(1.9)$ and $\Gamma^{1}$ is therefore asymptotic to this point. This leads to a more degenerate singular cycle in Fig. 8 with the orbit within $y<0$ connecting to the orange circle at $x=0$. This point is 'extra' singular due to the exponentials. Studying limit cycles in this case would require use of the same machinery used to describe the oscillations in the Le Corbeiller problem. One could also consider the termination of relaxation oscillations as $a \rightarrow 0^{+}$in the Le Corbellier system. The mechanism for termination is a (degenerate) singularly perturbed boundary-focus bifurcation [15], which occurs when the unstable focus of the limiting PWS system collides with the switching manifold $\Sigma$ for $a=0$. Unfolding this bifurcation requires additional blow-up transformations. In fact, the necessary transformations and singular geometry have been described in the $\mathrm{PhD}$ thesis [13, Ch. 6], where it was shown that similarly to the canard explosion phenomenon, there exists a family of singular cycles mediating a transition between large amplitude relaxation oscillations for $a=\mathcal{O}(1)$ and small amplitude oscillations for $a=\mathcal{O}(\epsilon)$. Unlike classical canard explosions, however, the cycles only grow as an algebraic 
(as opposed to exponential) function of $\epsilon$ with respect to variation in $a$; see also [14,15,24]. The actual onset of oscillations is due to a supercritical Hopf bifurcation in an $\epsilon$-dependent domain. The analysis presented in [13, Ch. 6] is purely singular, however, and a proof of persistence for $0<\epsilon \ll 1$ remains for future work.

Regarding the generality and extendability of the methods presented herein, it is worthy to note that a competition between rational/polynomial and exponential/logarithmic terms may be expected to lead to similar dynamical features even in the absence of an explicit perturbation parameter $\epsilon$. To see this, consider the very common case in which the term $e^{h(x)}$ with $h(x)=x$ arises in the right-hand-side. This term will dominate rational/polynomial contributions on large domains when $x \gg 1$, while the rational/polynomial terms dominate when $x \ll-1$. Hence, the system will 'look PWS' on a large domain. This can be formalised by rescaling $x=\epsilon^{-1} X$ for some $0<\epsilon \ll 1$, or by Poincaré compactification. Such systems can then be studied using the methods developed in this manuscript. It follows that the techniques presented herein have applicability for a significant and important class of dynamical systems in applications characterised by competing rational/polynomial and exponential/logarithmic terms. The adapted blow-up methods presented herein provide a rigorous and systematic method for connecting dynamical regimes that are well approximated by distinct limiting problems corresponding to rational/polynomial and exponential/logarithmic terms.

In conclusion, our geometric approach for studying the Hester and the Le Corbeiller systems is general and we therefore expect that it can be applied to different problems of this kind, including the ones discussed in the introduction. This will be part of future work in the area.

Acknowledgements MW was supported by the Australian Research Council DP180103022 grant. SJ would also like to thank the Technical University of Denmark (DTU) and the second author, for hospitality during their stay, without which this work may not have been possible.

Funding Open Access funding enabled and organized by Projekt DEAL.

Open Access This article is licensed under a Creative Commons Attribution 4.0 International License, which permits use, sharing, adaptation, distribution and reproduction in any medium or format, as long as you give appropriate credit to the original author(s) and the source, provide a link to the Creative Commons licence, and indicate if changes were made. The images or other third party material in this article are included in the article's Creative Commons licence, unless indicated otherwise in a credit line to the material. If material is not included in the article's Creative Commons licence and your intended use is not permitted by statutory regulation or exceeds the permitted use, you will need to obtain permission directly from the copyright holder. To view a copy of this licence, visit http://creativecommons.org/licenses/by/4.0/.

\section{References}

1. Bossolini, E., Brøns, M., Kristiansen, K.U.: Singular limit analysis of a model for earthquake faulting. Nonlinearity 30(7), 2805-2834 (2017)

2. Brøns, M.: Relaxation oscillations and canards in a nonlinear model of discontinuous plastic deformation in metals at very low temperatures. Proc. R. Soc. A Math. Phys. Eng. Sci. 461(2059), 2289-2302 (2005)

3. di Bernardo, M., Budd, C.J., Champneys, A.R., Kowalczyk, P.: Piecewise-Smooth Dynamical Systems: Theory and Applications. Springer, Berlin (2008)

4. Dieterich, J.H.: Time-dependent friction and mechanics of stick-slip. Pure Appl. Geophys. 116(4-5), 790-806 (1978)

5. Dieterich, J.H.: Modeling of rock friction. 1. Experimental results and constitutive equations. J. Geophys. Res. 84(NB5), 2161-2168 (1979)

6. Dumortier, F., Roussarie, R.: Canard cycles and center manifolds. Mem. Am. Math. Soc. 121, 1-96 (1996)

7. Ebers, J.J., Moll, J.L.: Large-signal behavior of junction transistors. Inst. Radio Eng. Convention Record 42(12), 1761-1772 (1954) 
8. Estrin, Y., Kubin, L.P.: Criterion for thermomechanical instability of low temperature plastic deformation. Scripta Metallurgica 14(12), 1359-1364 (1980)

9. Fenichel, N.: Geometric singular perturbation theory for ordinary differential equations. J. Diff. Eq. 31, 53-98 (1979)

10. Filippov, A.F.: Differential Equations with Discontinuous Righthand Sides. Mathematics and its Applications. Kluwer Academic Publishers (1988)

11. Hester, D.L.: The nonlinear theory of a class of transistor oscillators. IEEE Trans. Circuit Theory CT15(2), 111-118 (1968)

12. Hogan, S.J., Homer, M.E., Jeffrey, M.R., Szalai, R.: Piecewise smooth dynamical systems theory: the case of the missing boundary equilibrium bifurcations. 26, 1161-1173 (2016)

13. Jelbart, S.: Beyond slow-fast: Relaxation oscillations in singularly perturbed non-smooth systems. PhD thesis, The University of Sydney (2020)

14. Jelbart, S., Kristiansen, K.U., Wechselberger, M.: Singularly perturbed boundary-equilibrium bifurcations. arXiv:2103.09613, (March 2021)

15. Jelbart, S., Kristiansen, K.U., Wechselberger, M.: Singularly perturbed boundary-focus bifurcations. J. Differ. Equ. 296, 412-492 (2021)

16. Jelbart, S., Wechselberger, M.: Two-stroke relaxation oscillators. Nonlinearity 33, 2364-2408 (2020)

17. Jones, C.K.R.T.: Geometric Singular Perturbation Theory. Lecture Notes in Mathematics, Dynamical Systems Springer, Berlin, Heidelberg, 44-18 (1995)

18. Kosiuk, I., Szmolyan, P.: Geometric singular perturbation analysis of an autocatalator model. Discret. Contin. Dyn. Syst. Ser. S 2(4), 783-806 (2009)

19. Kosiuk, I., Szmolyan, P.: Geometric analysis of the Goldbeter minimal model for the embryonic cell cycle. J. Math. Biol. 72(5), 1337-1368 (2016)

20. Kristiansen, K.U.: Blowup for flat slow manifolds. Nonlinearity 30(5), 2138-2184 (2017)

21. Kristiansen, K.U.: A new type of relaxation oscillation in a model with rate-and-state friction. Nonlinearity 33, 2960-3037 (2020)

22. Kristiansen, K.U.: The regularized visible fold revisited. J. Nonlinear Sci. 30, 2463-2511 (2020)

23. Kristiansen, K.U., Hogan, S.J.: Resolution of the piecewise smooth visible-invisible two-fold singularity in R3 using regularization and blowup. J. Nonlinear Sci. 29(2), 723-787 (2018)

24. Kristiansen, K.U., Szmolyan, P.: Relaxation oscillations in substrate-depletion oscillators close to the nonsmooth limit. Nonlinearity 34, 1030-1083 (2021)

25. Krupa, M., Szmolyan, P.: Extending geometric singular perturbation theory to nonhyperbolic points - fold and canard points in two dimensions. SIAM J. Math. Anal. 33(2), 286-314 (2001)

26. Krupa, M., Szmolyan, P.: Relaxation oscillation and canard explosion. J. Differ. Equ. 174(2), 312-368 (2001)

27. Kuehn, C.: Multiple Time Scale Dynamics. Springer, Berlin (2015)

28. Kuznetsov, Yu.A, Rinaldi, S., Gragnani, A.: One parameter bifurcations in planar Filippov systems. Int. J. Bif. Chaos 13, 2157-2188 (2003)

29. Le Corbeiller, P.: Two-stroke oscillators. Ire Trans. Circuit Theory 7(4), 387-398 (1960)

30. Putelat, T., Dawes, J.H.P., Champneys, A.R.: A phase-plane analysis of localized frictional waves. Proc. R. Soc. A Math. Phys. Eng. Sci. 473(2203), (2017)

31. Rice, J.R., Lapusta, N., Ranjith, K.: Rate and state dependent friction and the stability of sliding between elastically deformable solids. J. Mech. Phys. Solids 49(9), 1865-1898 (2001)

32. Ruina, A.: Slip instability and state variable friction laws. J. Geophys. Res. 88(NB12), 359-370 (1983)

33. Woodhouse, J., Putelat, T., McKay, A.: Are there reliable constitutive laws for dynamic friction? Philos. Trans. R. Soc. A Math. Phys. Eng. Sci. 373(2051), 20140401 (2015)

Publisher's Note Springer Nature remains neutral with regard to jurisdictional claims in published maps and institutional affiliations. 